JOURNAL OF THE

AMERICAN MATHEMATICAL SOCIETY

Volume 24, Number 4, October 2011, Pages 1175-1226

S 0894-0347(2011)00706-6

Article electronically published on June 8, 2011

\title{
W*-SUPERRIGIDITY FOR BERNOULLI ACTIONS OF PROPERTY (T) GROUPS
}

\author{
ADRIAN IOANA
}

\section{Contents}

0. Introduction 1175

1. Preliminaries 1182

2. Rigid subalgebras of $\mathrm{II}_{1}$ factors arising from generalized Bernoulli actions

3. A spectral gap argument 1189

4. Lower bound on height 1194

5. A conjugacy result for subalgebras 1196

6. A dichotomy result for subalgebras 1199

7. Popa's conjugacy criterion for actions 1203

8. Strong rigidity for embeddings of $\mathrm{II}_{1}$ factors 1207

9. $\mathrm{W}^{*}$-superrigidity 1213

10. Further applications 1217

References 1224

\section{INTRODUCTION}

0.1. The group measure space construction of Murray and von Neumann associates to every probability measure preserving (p.m.p.) action $\Gamma \curvearrowright X$ of a countable group $\Gamma$ on a probability space $(X, \mu)$ a finite von Neumann algebra $L^{\infty}(X) \rtimes \Gamma$ 24. If the action is essentially free and ergodic, then this algebra is a $\mathrm{II}_{1}$ factor which contains $L^{\infty}(X)$ as a Cartan subalgebra. A central problem in the theory of von Neumann algebras is understading how much of the group action is "remembered" by its von Neumann algebra.

The main goal of this paper is to prove that, for a large, natural family of group actions (Bernoulli actions of property $(\mathrm{T})$ groups), their associated $\mathrm{II}_{1}$ factor completely remembers the group and the action.

We start by giving some motivation for this result.

By a celebrated result of $\mathrm{A}$. Connes, if $\Gamma$ is infinite amenable, then the $\mathrm{II}_{1}$ factor of any free ergodic p.m.p. action $\Gamma \curvearrowright X$ is isomorphic to the hyperfinite $\mathrm{II}_{1}$ factor (4], see also [26] and [7]).

Received by the editors November 30, 2010 and, in revised form, April 20, 2011.

2010 Mathematics Subject Classification. Primary 46L36; Secondary 28D15, 37A20.

The author was supported by a Clay Research Fellowship.

(C)2011 American Mathematical Society Reverts to public domain 28 years from publication 
In contrast, the study of group measure space algebras arising from actions of non-amenable groups has led to a deep rigidity theory (see the survey [38] and the introduction of [42]).

In particular, S. Popa's seminal work [33, 34] shows that, for actions belonging to a large class, isomorphism of their group measure space algebras implies conjugacy of the actions. More precisely, assume that $\Gamma$ is an ICC (infinite conjugacy class) group which has an infinite, normal subgroup with the relative property $(\mathrm{T})$ and let $\Gamma \curvearrowright X$ be a free ergodic action. Additionally, suppose that $\Lambda \curvearrowright Y=Y_{0}^{\Lambda}$ is a Bernoulli action. Popa proves that if the actions $\Gamma \curvearrowright X$ and $\Lambda \curvearrowright Y$ are $\mathrm{W}^{*}$-equivalent, i.e. if they produce isomorphic von Neumann algebras (also known as $\mathrm{W}^{*}$-algebras), then the actions are conjugate 34. This means that $\Gamma \cong \Lambda$ and there exists an isomorphism of probability spaces $\theta: X \rightarrow Y$ such that $\theta \Gamma \theta^{-1}=\Lambda$.

The natural question underlying and motivating this result, explicitly formulated in the introduction of [34, is whether the same is true if one imposes all the conditions on only one of the actions. In other words, assume that $\Gamma$ is an ICC group having an infinite, normal subgroup with the relative property $(\mathrm{T})$ and suppose that $\Gamma \curvearrowright X=X_{0}^{\Gamma}$ is a Bernoulli action. Is it true that any free ergodic p.m.p. action $\Lambda \curvearrowright Y$ which is $\mathrm{W}^{*}$-equivalent to $\Gamma \curvearrowright X$, must be conjugate to it?

Further evidence that the answer to this question is true was provided by Popa. In [37, 34, he proves that this is the case if the actions are assumed to be orbit equivalent $(\mathrm{OE})$. This amounts to the existence of an isomorphism of probability spaces $\theta: X \rightarrow Y$ satisfying $\theta(\Gamma x)=\Lambda(\theta x)$, for almost every $x \in X$. Recall that orbit equivalence is stronger than $\mathrm{W}^{*}$-equivalence of actions. More precisely, as first noticed in [43. (see also [13]), two actions $\Gamma \curvearrowright X$ and $\Lambda \curvearrowright Y$ are orbit equivalent if and only if there exists an isomorphism of their group measure space algebras, $L^{\infty}(X) \rtimes \Gamma \cong L^{\infty}(Y) \rtimes \Lambda$, which identifies the Cartan subalgebras $L^{\infty}(X)$ and $L^{\infty}(Y)$.

0.2. The main result of this paper answers affirmatively the above question. Before stating it, we first review a few concepts and then introduce some terminology.

An inclusion $\left(\Gamma_{0} \subset \Gamma\right)$ of countable groups has the relative property $(T)$ of Kazhdan-Margulis if any unitary representation of $\Gamma$ which has almost invariant vectors must have a non-zero $\Gamma_{0}$-invariant vector [20, 23]. When $\Gamma_{0}=\Gamma$, this condition is equivalent to the property $(T)$ of the group $\Gamma$. Examples of relative property ( $\mathrm{T}$ ) inclusions of groups are given by $\left(\mathbb{Z}^{2} \subset \mathbb{Z}^{2} \rtimes \Gamma\right)$, for any non-amenable subgroup $\Gamma$ of $\mathrm{SL}_{2}(\mathbb{Z})\left[3\right.$, and by $\left(\Gamma_{0} \subset \Gamma_{0} \times \Gamma_{1}\right.$ ), for a property (T) group $\Gamma_{0}$ (e.g. $\left.\Gamma_{0}=\mathrm{SL}_{n}(\mathbb{Z}), n \geq 3\right)$ and an arbitrary countable group $\Gamma_{1}[20]$ ). For a probability space $\left(X_{0}, \mu_{0}\right)$, the Bernoulli action $\Gamma \curvearrowright\left(X_{0}^{\Gamma}, \mu_{0}^{\Gamma}\right)$ is given by $\gamma \cdot\left(x_{g}\right)_{g}=\left(x_{\gamma^{-1}}\right)_{g}$, for all $\left(x_{g}\right)_{g \in \Gamma} \in X_{0}^{\Gamma}$ and $\gamma \in \Gamma$.

In this paper, by a $\mathrm{W}^{*}$ - or $\mathrm{OE}-$ rigidity result we mean a result proving that two actions $\Gamma \curvearrowright X, \Lambda \curvearrowright Y$, which are $\mathrm{W}^{*}-$ or $\mathrm{OE}$-equivalent, must be conjugate. If this happens when only one of these actions is in a fixed class, while the other can be any free ergodic action of any countable group, we have a superrigidity result.

Theorem A ( $\mathbf{W}^{*}$-superrigidity). Let $\Gamma$ be a countable ICC group which admits an infinite normal subgroup $\Gamma_{0}$ such that the inclusion $\left(\Gamma_{0} \subset \Gamma\right)$ has the relative property $(T)$. Let $\left(X_{0}, \mu_{0}\right)$ be a non-trivial probability space and let $\Gamma \curvearrowright(X, \mu)=$ $\left(X_{0}^{\Gamma}, \mu_{0}^{\Gamma}\right)$ be the Bernoulli action. Denote $M=L^{\infty}(X) \rtimes \Gamma$ and let $p \in M$ be a 
projection. Let $\Lambda \curvearrowright(Y, \nu)$ be a free ergodic p.m.p. action of a countable group $\Lambda$. Denote $N=L^{\infty}(Y) \rtimes \Lambda$.

If $N \cong p M p$, then $p=1, \Gamma \cong \Lambda$ and the actions $\Gamma \curvearrowright X, \Lambda \curvearrowright Y$ are conjugate.

Moreover, any $*$-isomorphism $\theta: N \rightarrow M$ comes from a conjugacy of the actions $\Gamma \curvearrowright X, \Lambda \curvearrowright Y$ and a character of $\Gamma$ (see Theorem 9.1). Note that Theorem A provides a new, large class of $\mathrm{II}_{1}$ factors which are not group measure space factors.

Theorem A is proven in the framework of Popa's deformation/rigidity theory by playing against each other the "rigidity" of $\Gamma$ (manifested here in the form of the relative property (T)) and the "deformation properties" of Bernoulli actions $\Gamma \curvearrowright X$ (see Section 1.5).

Before discussing its method of proof in more detail, let us put it into context.

0.3. Despite remarkable progress in both the areas of $\mathrm{W}^{*}-$ and $\mathrm{OE}$-rigidity during the last decade (see [38, [12, [42]), $\mathrm{W}^{*}$-superrigidity results remained elusive until very recently ([31, [42]). Prior to these results, several large classes of OEsuperrigid actions were found ([11, 37], 39, [21], 15], 22]). In addition, for other classes of actions $\Gamma \curvearrowright X$, it was shown that $L^{\infty}(X)$ is the unique group measure space Cartan subalgebra of $L^{\infty}(X) \rtimes \Gamma$, up to unitary conjugacy ([28, ,31]). However, since no examples of actions having both of these properties were known, $\mathrm{W}^{*}$-superrigidity still could not be concluded.

The situation changed starting with the work of J. Peterson who was able to show the existence of virtually $\mathrm{W}^{*}$-superrigid actions (31]). He considered profinite actions of certain groups having infinite subgroups with the relative property $(\mathrm{T})$ and used, among other things, techniques and results from [30, 28, 15].

Shortly after, S. Popa and S. Vaes discovered the first concrete families of $\mathrm{W}^{*-}$ superrigid actions $([42])$. Furthermore, they succeeded in proving sweeping $\mathrm{W}^{*}-$ superrigidity results.

First, they showed that for groups $\Gamma$ in a certain class $\mathcal{G}$ of amalgamated free product groups, the $\mathrm{II}_{1}$ factor of any free ergodic action $\Gamma \curvearrowright X$ has a unique group measure space Cartan subalgebra.

Then, by applying the OE-superrigidity results from [37, 39] and 22], they respectively deduced that the following actions are $\mathrm{W}^{*}$-superrigid: a) Bernoulli actions, generalized Bernoulli actions, Gaussian actions of groups $\Gamma \in \mathcal{G}$ and b) any free mixing action of $\Gamma=\mathrm{PSL}_{n}(\mathbb{Z}) *_{T_{n}} \mathrm{PSL}_{n}(\mathbb{Z}) \in \mathcal{G}$, where $T_{n} \subset \mathrm{PSL}_{n}(\mathbb{Z})$ is the subgroup of upper triangular matrices and $n \geq 3$.

The class $\mathcal{G}$ consists of groups of the form $\Gamma=\Gamma_{1} *_{\Sigma} \Gamma_{2}$ subject to several conditions: 1) $\Sigma$ is amenable, 2) $\Sigma$ is "almost malnormal" in $\Gamma_{1}$, normal in $\Gamma_{2}$ and 3) $\Gamma_{1}$ is "rigid" (e.g. has property $(\mathrm{T})$ or is a product of non-amenable groups). As is easy to see, 2) precludes groups $\Gamma \in \mathcal{G}$ from having an infinite normal subgroup with the relative property $(\mathrm{T})$. Therefore, Theorem A and the results of [42] cover disjoint classes of groups. In particular, Theorem $\mathrm{A}$ is the first $\mathrm{W}^{*}$-superrigidity result that applies to property $(\mathrm{T})$ groups.

0.4. In this paper, we introduce a general strategy for analyzing group measure space decompositions of $\mathrm{II}_{1}$ factors and implement it successfully to derive Theorem A. To outline our approach, let $M$ be the $\mathrm{II}_{1}$ factor associated to some fixed, "known" action $\Gamma \curvearrowright X$.

The first part consists of "classifying" all unital $*$-homomorphisms $\theta: M \rightarrow$ $M \bar{\otimes} M$ (or embeddings of $M$ into $M \bar{\otimes} M$ ) in terms of the decomposition $M=$ 
$L^{\infty}(X) \rtimes \Gamma$ (i.e., one would like to have a rigidity result concerning the structure of such $\theta$ (see Thm. C)).

Now, suppose that $M$ also arises as the $\mathrm{II}_{1}$ factor of a "mystery" action $\Lambda \curvearrowright Y$.

The decomposition of $M$ as $L^{\infty}(Y) \rtimes \Lambda$ induces a unital $*$-homomorphism $\theta$ : $M \rightarrow M \bar{\otimes} M$ given by $\theta\left(a v_{\lambda}\right)=a v_{\lambda} \otimes v_{\lambda}$, for all $a \in L^{\infty}(Y)$ and every $\lambda \in \Lambda$. This embedding of $M$ into $M \bar{\otimes} M$ has been introduced and used by Popa and Vaes in the proof of 42, Lemma 3.2.

In the second part, we apply the above classification to $\theta$. Thus, we have some information about the form of $\theta$ with respect to both group measure space decompositions of $M$. This relates the two decompositions and, ideally, the relationship will be powerful enough to imply that the two decompositions coincide, up to unitary conjugacy. If in addition we know that the action $\Gamma \curvearrowright X$ is OE-superrigid, then we can deduce that the involved actions must be conjugate.

Remark. Using this strategy it can be readily seen that if a $\mathrm{II}_{1}$ factor $M$ has property $(\mathrm{T})$, in the sense of Connes-Jones [10, then it admits only countably many group measure space decompositions (see Section 10).

0.5. Following the above strategy, we prove that the $\mathrm{II}_{1}$ factor $M=L^{\infty}(X) \rtimes \Gamma$ defined in Theorem A has a unique group measure space Cartan subalgebra. In combination with Popa's OE superrigidity result [37 this proves Theorem A.

To apply our approach, we first need to describe all $*$-homomorphisms $\theta: M \rightarrow$ $M \bar{\otimes} M$. We start by addressing the seemingly easier question of describing all $*-$ homomorphisms $\theta: M \rightarrow M$. In this context, we prove a strong rigidity result in the spirit of 34 .

Theorem B. Let $M=L^{\infty}(X) \rtimes \Gamma$ be as in Theorem A. Let $\theta: M \rightarrow M$ be a (not necessarily unital) $*$-homomorphism and assume that $\Gamma$ is torsion free.

Then one of the following holds true:

(1) $\theta(M) \prec_{M} L(\Gamma)$ or

(2) $\theta$ is unital and we can find a character $\eta$ of $\Gamma$, a morphism $\delta: \Gamma \rightarrow \Gamma$ and a unitary $u \in M$ such that $u \theta\left(L^{\infty}(X)\right) u^{*} \subset L^{\infty}(X)$ and $u \theta\left(u_{\gamma}\right) u^{*}=\eta(\gamma) u_{\delta(\gamma)}$, for all $\gamma \in \Gamma$.

Furthermore, generalizing the main result of [34] (in the case of w-rigid groups), we prove a rigidity result for $*$-homomorphisms between two $\mathrm{II}_{1}$ factors, each of which share some, but not all, of the defining properties of $M$ (see Theorem 8.1).

Before elaborating on conditions (1) and (2), let us recall the notation used in Theorem B. Thus, we denote by $L(\Gamma) \subset M$ the copy of the group von Neumann algebra of $\Gamma$ generated by the canonical unitaries $\left\{u_{\gamma}\right\}_{\gamma \in \Gamma} \subset M$. Also, we use the notation $Q \prec_{M} B$ to indicate that "a corner of a subalgebra $Q \subset M$ can be embedded into a subalgebra $B \subset M$ inside a factor $M$ ", in the sense of Popa (33], see 1.3.1). This roughly means that we can conjugate $Q$ into $B$ with a unitary element from $M$.

Now, condition (1) essentially says that $\theta$ comes from an embedding of $M$ into $L(\Gamma)$.

To better explain condition (2), we rephrase it differently using the following example.

Example. Quotient actions naturally induce $*$-homomorphisms between group measure space algebras. 
Recall that an action $\Gamma \curvearrowright Y$ is a quotient (or a factor) of an action $\Gamma \curvearrowright Z$ if there exists a $\Gamma$-equivariant, measure preserving map $q: Z \rightarrow Y$. This is equivalent to the existence of a $*$-homomorphism $\theta: L^{\infty}(Y) \rtimes \Gamma \rightarrow L^{\infty}(Z) \rtimes \Gamma$ such that $\theta\left(L^{\infty}(Y)\right) \subset L^{\infty}(Z)$ and $\theta\left(u_{\gamma}\right)=u_{\gamma}$, for all $\gamma \in \Gamma$.

With this in mind, (2) says that there exists a subgroup $\Gamma^{\prime}$ of $\Gamma$ isomorphic to $\Gamma$ such that $\theta$ comes from a conjugacy between $\Gamma \curvearrowright X$ and a quotient of the action $\Gamma^{\prime} \curvearrowright X$.

0.6. Next, returning to our initial question of describing $*$-homomorphisms $\theta$ : $M \rightarrow M \bar{\otimes} M$, we prove the following classification result.

Theorem C. Let $M=L^{\infty}(X) \rtimes \Gamma$ be as in Theorem A. Let $\theta: M \rightarrow M \bar{\otimes} M$ be a (not necessarily unital) $*$-homomorphism and assume that $\Gamma$ is torsion free.

Then one of the following holds true:

(1) $\theta\left(L\left(\Gamma_{0}\right)\right) \prec_{M \bar{\otimes} M} L(\Gamma) \otimes 1$ or $\theta\left(L\left(\Gamma_{0}\right)\right) \prec_{M \bar{\otimes} M} 1 \otimes L(\Gamma)$.

(2) $\theta(M) \prec_{M \bar{\otimes} M} L(\Gamma) \bar{\otimes} M$ or $\theta(M) \prec_{M \bar{\otimes} M} M \bar{\otimes} L(\Gamma)$.

(3) $\theta$ is unital and we can find a character $\eta$ of $\Gamma$, two group morphisms $\delta_{1}, \delta_{2}$ : $\Gamma \rightarrow \Gamma$ and a unitary $u \in M$ such that $u \theta\left(L^{\infty}(X)\right) u^{*} \subset L^{\infty}(X) \bar{\otimes} L^{\infty}(X)$ and $u \theta\left(u_{\gamma}\right) u^{*}=\eta(\gamma)\left(u_{\delta_{1}(\gamma)} \otimes u_{\delta_{2}(\gamma)}\right)$, for all $\gamma \in \Gamma$.

This result holds true without the torsion freeness assumption, but in this case its statement becomes considerably more complicated (see Theorem 8.2). Therefore, for simplicity, we assume only here that $\Gamma$ is torsion free although we will later state and prove Theorem $\mathrm{C}$ in full generality. This generalization will enable us to prove Theorem A for groups with torsion, including the interesting examples $\mathrm{SL}_{n}(\mathbb{Z})(n \geq 3)$ and $\mathbb{Z}^{2} \rtimes \mathrm{SL}_{2}(\mathbb{Z})$.

0.7. In Section 9, we will combine Theorem $\mathrm{C}$ and the strategy described in 0.4 to deduce Theorem A. Since the argument needed is quite involved, we will not elaborate more on this here. Rather, we mention a few words on the proofs of Theorems B and C. Since these proofs are analogous, let us only discuss the simpler case of Theorem B.

Consider, for simplicity, a unital embedding $\theta$ of $M=L^{\infty}(X) \rtimes \Gamma$ into itself. The starting point of the proof of Theorem B is Popa's "absorption" result for relative property (T) subalgebras of $M$ which enables us to assume that $\theta(L(\Gamma)) \subset L(\Gamma)$ (33).

The main ingredient of the proof consists of the following dichotomy for abelian subalgebras of $\mathrm{II}_{1}$ factors coming from Bernoulli actions.

Theorem D. Let $\Gamma \curvearrowright(X, \mu)$ be a Bernoulli action of a countable group $\Gamma$. Denote $M=L^{\infty}(X) \rtimes \Gamma$ and let $D \subset M$ be a unital abelian von Neumann subalgebra. Suppose that there exists a sequence of unitaries $\left\{u_{n}\right\}_{n \geq 1} \subset L(\Gamma)$ such that $u_{n} \rightarrow 0$ (weakly) and $u_{n}$ normalizes $D$ (i.e. $u_{n} D u_{n}^{*}=D$ ), for all $n \geq 1$. Then either:

(1) $D \prec_{M} L(\Gamma)$ or

(2) $D^{\prime} \cap M$ is of type $I$ and there exists a unitary $u \in M$ such that $u L^{\infty}(X) u^{*} \subset$ $D^{\prime} \cap M$.

The proof of Theorem D is based on several new ideas as well as on an idea of J. Peterson ([31], see Section 3).

Let us emphasize two key tools that we introduce, both of which are related to a certain notion of "height". 
First, fix an element $x$ in a group von Neumann algebra $L(\Gamma)$ and write $x=$ $\sum_{\gamma \in \Gamma} x_{\gamma} u_{\gamma}$, where $x_{\gamma} \in \mathbb{C}$. The height of $x$ is defined as $h(x)=\max _{\gamma \in \Gamma}\left|x_{\gamma}\right|$ and measures the distance between $x$ and the group $\Gamma=\left\{u_{\gamma}\right\}_{\gamma \in \Gamma} \subset L(\Gamma)$. With this notation, we show that if condition (1) of Theorem D fails, then $\inf _{n} h\left(u_{n}\right)>0$. Roughly, this means that the $u_{n}$ 's are uniformly close to $\Gamma$. This fact is crucial because it allows us to carry subsequent calculations by analogy with the case when $u_{n} \in \Gamma$.

More generally, if $x=\sum_{g \in \Gamma} x_{g} u_{g}$ is an element of a crossed product algebra $M=A \rtimes \Gamma$, then we define its height over $A$ as $h_{A}(x)=\max _{g \in \Gamma}\left\|x_{g}\right\|_{2}$. Our second tool is a new "intertwining theorem" for subalgebras $D \subset M$. Thus, we prove that if the height function $h_{A}$ is bounded from below on the unitary group of $D$, i.e. $\inf _{x \in \mathcal{U}(D)} h_{A}(x)>0$, then a corner of $D$ can be embedded into $A$ inside $M$ (see Theorem 1.3.2).

Returning to the explanation of Theorem B, fix a sequence $\left\{\gamma_{n}\right\}_{n \geq 1} \subset \Gamma$ with $\gamma_{n} \rightarrow \infty$. Notice that the unitary elements $u_{n}=\theta\left(u_{\gamma_{n}}\right)$ belong to $L(\Gamma)$ and normalize $D=\theta\left(L^{\infty}(X)\right)$. By applying Theorem D we derive that either (1) $D \prec_{M} L(\Gamma)$ or $(2) D^{\prime} \cap M=u L^{\infty}(X) u^{*}$, for some unitary $u$ (this is true only up to finite index).

In the first case, since $\theta(L(\Gamma)) \subset L(\Gamma)$, we deduce that $\theta(M) \prec_{M} L(\Gamma)$; thus condition (1) in the conclusion of Theorem B is satisfied.

In the second case, denote by $\tilde{\Gamma}$ the group $\left\{\theta\left(u_{\gamma}\right)\right\}_{\gamma \in \Gamma}$. Then $\tilde{\Gamma}$ normalizes $\tilde{D}=$ $D^{\prime} \cap M$. The crossed product algebra $N=\tilde{D} \rtimes \tilde{\Gamma}$, viewed inside $M=L^{\infty}(X) \rtimes \Gamma$, has the property that its core, $\tilde{D}$, is a unitary conjugate of $L^{\infty}(X)$ in addition to satisfying $L(\tilde{\Gamma}) \subset L(\Gamma)$. A generalization of Popa's conjugacy criterion for actions ([34], see Section 7) implies that we may in fact assume that $\tilde{\Gamma} \subset \Gamma$ (modulo scalars). In other words, condition (2) in the conclusion of Theorem B holds true.

0.8. We continue with an application of Theorem B.

Corollary E. Let $\Gamma \curvearrowright(X, \mu)$ be a non-trivial Bernoulli action of $\Gamma=\mathbb{F}_{m} \times \mathbb{F}_{n}$, with $2 \leq m, n \leq \infty$. Denote $M=L^{\infty}(X) \rtimes \Gamma$. If $\theta: M \rightarrow p M p$ is a unital $*$-homomorphism, for some projection $p \in M$, then $p=1$. Moreover, there exist a character $\eta$ of $\Gamma$, a group morphism $\delta: \Gamma \rightarrow \Gamma$ and a unitary $u \in M$ such that $u \theta\left(L^{\infty}(X)\right) u^{*} \subset L^{\infty}(X)$ and $u \theta\left(u_{\gamma}\right) u^{*}=\eta(\gamma) u_{\delta(\gamma)}$, for all $\gamma \in \Gamma$.

The proof of Corollary E is the combination of two facts: an extension of Theorem B to groups $\Gamma$ that are products of non-amenable groups (see Theorem 8.1) and a result of N. Ozawa and S. Popa guaranteeing that, if $\Gamma$ is non-amenable and has the complete metric approximation property (e.g. if $\Gamma=\mathbb{F}_{m} \times \mathbb{F}_{n}$ ), then there is no embedding of $M$ into $L(\Gamma)$ ([28]).

Corollary E provides the first examples of $\mathrm{II}_{1}$ factors $M$ which do not embed into any of their corners. (The question of whether such factors exist was posed by N. Ozawa during a seminar at UCLA in 2007.)

Corollary E also reduces the calculation of the endomorphism semigroup of $M$ (i.e., the semigroup, $\operatorname{End}(M)$, of unital $*$-homomorphisms $\theta: M \rightarrow M$ ) to an ergodic-theoretic problem. More precisely, any $\theta \in \operatorname{End}(M)$ is determined by the following data: a character $\eta$ of $\Gamma$, a morphism $\delta: \Gamma \rightarrow \Gamma$ and a measure preserving map $q: X \rightarrow X$ satisfying $q(\delta(\gamma) x)=\gamma q(x)$, for every $\gamma \in \Gamma$ and almost all $x \in X$ (see the example from Section 0.5). 
0.9. Finally, let us present an application of Theorem C.

After introducing the group measure space construction in 24, Murray and von Neumann later found a simpler way of constructing $\mathrm{II}_{1}$ factors. Thus, to every countable group $\Lambda$, one can associate its group von Neumann algebra, $L(\Lambda)([25])$. This algebra is finite in general and it is a $\mathrm{II}_{1}$ factor if and only if $\Lambda$ is ICC.

The first examples of $\mathrm{II}_{1}$ factors which are not group von Neumann algebras were discovered by A. Connes (4], see also [18]). Recently, more examples have been exhibited in [17] and 40. All of these examples were obtained by analyzing antiautomorphisms. More precisely, one first proves that the $\mathrm{II}_{1}$ factors involved either do not have anti-automorphisms (44, 17, 40]) or do not have anti-automorphisms of order $2([18])$. Then, since any group von Neumann algebra $L(\Lambda)$ admits an antiautomorphism of order 2 (given by $\Phi\left(v_{\lambda}\right)=v_{\lambda^{-1}}$, for $\lambda \in \Lambda$ ), one deduces lack of group von Neumann algebra decomposition.

As a consequence of Theorem $\mathrm{C}$, we obtain a wide class of new examples through a different method.

Corollary F. Let $M=L^{\infty}(X) \rtimes \Gamma$ be as in Theorem A. Assume that $\Gamma$ is torsion free. Then, for any projection $p \in M \backslash\{0,1\}$, the $I I_{1}$ factor $p M p$ is not isomorphic to the group von Neumann algebra, $L(\Lambda)$, of a countable group $\Lambda$.

For a more general statement, see Theorem 10.1.

Corollary $\mathrm{F}$ provides in particular the first examples of $\mathrm{II}_{1}$ factors which have involutary anti-automorphisms and yet are not group von Neumann algebras (see Remark 10.3). This answers a question posed by V.F.R. Jones in [18, Remark 5.7.

The proof of Corollary $\mathrm{F}$ borrows its main idea from the strategy described in Section 0.4. If $p M p=L(\Lambda)$, then $\theta: p M p \rightarrow p M p \bar{\otimes} p M p$ given by $\theta\left(v_{\lambda}\right)=v_{\lambda} \otimes v_{\lambda}$ is a unital $*$-homomorphism. By taking amplifications, we get a $*$-homomorphism $\tilde{\theta}: M \rightarrow M \bar{\otimes} M$ with $\tau(\tilde{\theta}(1))=\tau(p)$. It is immediate to see that $\tilde{\theta}$ does not satisfy conditions (1) and (2) of Theorem C.

Therefore, $\tilde{\theta}$ is forced to be unital and hence $p=1$.

Furthermore, as we explain in Section 10 (IV), an extension of our results can be used to show that the $\mathrm{II}_{1}$ factors $p M p$ from Corollary $\mathrm{F}$ are in fact not isomorphic to any twisted group von Neumann algebra $L_{\alpha}(\Lambda)$.

Corollary G. Let $M=L^{\infty}(X) \rtimes \Gamma$ be as in Theorem A. Assume that $\Gamma$ is torsion free. Then, given a projection $p \in M \backslash\{0,1\}$, the $I I_{1}$ factor $p M p$ is not isomorphic to $L_{\alpha}(\Lambda)$, for any countable group $\Lambda$ and any 2 -cocycle $\alpha \in H^{2}(\Lambda, \mathbb{T})$.

Organization of the paper. Besides the introduction, this paper has ten other sections. In Section 1, we recall a few notions and results regarding von Neumann algebras and establish a crucial intertwining result (Theorem 1.3.2). In Section 2, we prove an "absorption" result for relative property $(\mathrm{T})$ subalgebras of $\mathrm{II}_{1}$ factors coming from generalized Bernoulli actions. The proof of Theorem D occupies Sections 3-6. Further, in Section 7, we generalize Popa's conjugacy criterion for actions. In Section 8, we combine the results of the previous sections to derive Theorems B and C, while in Section 9 we deduce Theorem A. Our last section is devoted to several applications of Theorems B and C (including Corollaries E, F and $\mathrm{G})$. 


\section{Preliminaries}

1.1. Finite von Neumann algebras. We first review a few concepts and constructions involving von Neumann algebras (see e.g. [2]). In this paper we work with finite von Neumann algebras $M$ always endowed with a fixed faithful normal tracial state $\tau$.

We denote by $L^{2}(M)$ the Hilbert space obtained by completing $M$ with respect to the 2 -norm $\|x\|_{2}=\tau\left(x^{*} x\right)^{\frac{1}{2}}$. Hereafter, we view every $x \in M$ both as an element of $L^{2}(M)$ and as a bounded (left multiplication) operator on $L^{2}(M)$. The inequality $\|a x b\|_{2} \leq\|a\|\|\mid\| x\left\|_{2}\right\| b \|$, for all $a, b, x \in M$, will be used often.

We denote by $\mathcal{U}(M)$ the group of unitaries of $M$, by $\operatorname{Aut}(M)$ the group of automorphisms of $M$ endowed with the pointwise $\|\cdot\|_{2}$ topology and by $\operatorname{id}_{M}$ the identity automorphism of $M$. For every $u \in \mathcal{U}(M)$, the inner automorphism $\operatorname{Ad}(u)$ of $M$ is given by $\operatorname{Ad}(u)(x)=u x u^{*}$. We also denote by $\mathcal{P}(M)$ the set of projections of $M$, by $\mathcal{Z}(M)$ the center of $M$ and by $(M)_{1}$ the set of $x \in M$ with $\|x\| \leq 1$. A Hilbert space $\mathcal{H}$ is called an $M$-bimodule if it is endowed with commuting left and right Hilbert $M$-module structures.

For a subset $X$ of $M$, we denote by $L^{2}(X)$ its closure in $L^{2}(M)$, by $X^{\prime} \cap M$ its commutant in $M$ and by $X^{\prime \prime}$ the von Neumann algebra it generates.

Let $B$ be a unital von Neumann subalgebra of $(M, \tau)$. We denote by $q N_{M}(B)$ the quasi-normalizer of $B$ in $M$, i.e., the set of $x \in M$ for which there exist $x_{1}, \ldots, x_{n} \in M$ satisfying $x B \subset \sum_{i=1}^{n} B x_{i}, B x \subset \sum_{i=1}^{n} x_{i} B$ (see [35]).

Note that $q N_{M}(B)$ contains $\mathcal{N}_{M}(B)$, the normalizer of $B$ in $M$, i.e., the set of unitaries $u \in M$ such that $\operatorname{Ad}(u)(B)=B$. If $q \mathcal{N}_{M}(B)^{\prime \prime}=M$, we say that $B$ is quasi-regular in $M$ and if $\mathcal{N}_{M}(B)^{\prime \prime}=M$, we say that $B$ is regular in $M$. If $\Gamma_{0} \subset \Gamma$ is an almost normal inclusion of countable groups, then $L\left(\Gamma_{0}\right)$ is quasi-regular in $L(\Gamma)$.

Recall that if $e_{B}$ is the orthogonal projection from $L^{2}(M)$ onto $L^{2}(B)$, then its restriction to $M$ is equal to $E_{B}$, the conditional expectation from $M$ onto $B$. Jones' basic construction $\left\langle M, e_{B}\right\rangle$ is the von Neumann algebra generated by $M$ and $e_{B}$ inside $\mathbb{B}\left(L^{2}(M)\right)$. The basic construction $\left\langle M, e_{B}\right\rangle$ contains the span of $\left\{x e_{B} y \mid x, y \in M\right\}$ as a dense $*$-subalgebra and is endowed with a faithful normal semi-finite trace $\operatorname{Tr}$ given by $\operatorname{Tr}\left(x e_{B} y\right)=\tau(x y)$. We denote by $L^{2}\left(\left\langle M, e_{B}\right\rangle\right)$ the associated Hilbert space.

Next, let $\omega$ be a free ultrafilter on $\mathbb{N}$. For a finite von Neumann algebra $(M, \tau)$, we denote by $\left(M^{\omega}, \tau_{\omega}\right)$ its ultrapower algebra, i.e., the finite von Neumann algebra $\ell^{\infty}(\mathbb{N}, M) / \mathcal{I}$, where the trace $\tau_{\omega}$ is given by $\tau_{\omega}\left(\left(x_{n}\right)_{n}\right)=\lim _{n \rightarrow \omega} \tau\left(x_{n}\right)$ and $\mathcal{I}$ is the ideal of $x=\left(x_{n}\right)_{n} \in \ell^{\infty}(\mathbb{N}, M)$ such that $\tau_{\omega}\left(x^{*} x\right)=0$. Note that $M$ embeds into $M^{\omega}$ through the map $x \rightarrow\left(x_{n}\right)_{n}$, where $x_{n}=x$, for all $n$, and that any automorphism $\theta$ of $M$ extends to an automorphism of $M^{\omega}$ by letting $\theta\left(\left(x_{n}\right)_{n}\right)=$ $\left(\theta\left(x_{n}\right)\right)_{n}$, for all $x=\left(x_{n}\right) \in M^{\omega}$.

Finally, let $M$ be a $\mathrm{II}_{1}$ factor and $t>0$. Let $n \geq t$ be an integer and $p \in \mathbb{M}_{n}(\mathbb{C}) \otimes$ $M$ be a projection of normalized trace $\frac{t}{n}$. The isomorphism class of the algebra $p\left(\mathbb{M}_{n}(\mathbb{C}) \otimes M\right) p$ is independent of the choice of $n$ and $p$, is called the $t$-amplification of $M$ and is denoted by $M^{t}$. If $N$ is a $\mathrm{II}_{1}$ factor and $\theta: N \rightarrow M$ is a unital $*$-homomorphism, then for every $t>0$ there exists a natural $*$-homomorphism $\theta^{t}: N^{t} \rightarrow M^{t}$. Moreover, $\theta^{t}$ is uniquely defined, up to composition with an inner automorphism. 
1.2. The crossed product construction. Let $\sigma: \Gamma \rightarrow \operatorname{Aut}(B)$ be a trace preserving action of a countable group $\Gamma$ on a finite von Neumann algebra $(B, \tau)$ ([24]). Set $\mathcal{H}=L^{2}(B) \bar{\otimes} \ell^{2}(\Gamma)$ and for every $b \in B, \gamma \in \Gamma$, define the operators $L_{f}, u_{\gamma} \in \mathbb{B}(\mathcal{H})$ through the formulas

$$
L_{b}\left(b^{\prime} \otimes \delta_{\gamma^{\prime}}\right)=b b^{\prime} \otimes \delta_{\gamma^{\prime}}, \quad u_{\gamma}\left(b^{\prime} \otimes \delta_{\gamma^{\prime}}\right)=\sigma(\gamma)\left(b^{\prime}\right) \otimes \delta_{\gamma \gamma^{\prime}}, \quad \forall b^{\prime} \in L^{2}(B), \gamma^{\prime} \in \Gamma .
$$

Since $u_{\gamma} u_{\gamma^{\prime}}=u_{\gamma \gamma^{\prime}}, L_{b} L_{b^{\prime}}=L_{b b^{\prime}}, u_{\gamma} L_{b} u_{\gamma}^{*}=L_{\sigma(\gamma)(b)}$, for every $\gamma, \gamma^{\prime} \in \Gamma$ and $b, b^{\prime} \in B$, the linear span of $\left\{L_{b} u_{\gamma} \mid b \in B, \gamma \in \Gamma\right\}$ is a $*$-subalgebra of $\mathbb{B}(\mathcal{H})$. The strong operator closure of this algebra, denoted $B \rtimes_{\sigma} \Gamma$, is called the crossed product von Neumann algebra associated to $\sigma$. The trace $\tau$ extends to a trace on $B \rtimes_{\sigma} \Gamma$ given by $\tau\left(b u_{\gamma}\right)=\delta_{\gamma, e} \tau(b)$, making $B \rtimes_{\sigma} \Gamma$ a finite von Neumann algebra. Every element $x \in B \rtimes_{\sigma} \Gamma$ decomposes as $x=\sum_{\gamma \in \Gamma} b_{\gamma} u_{\gamma}$, where the convergence holds in $\|.\|_{2}$ and $b_{\gamma} \in B$, for every $\gamma \in \Gamma$.

Two important examples of crossed product algebras arise when $B$ is either trivial or abelian.

Firstly, if $B=\mathbb{C} 1$ (with $\Gamma$ acting trivially), then the associated crossed product algebra is precisely the group von Neumann algebra $L(\Gamma)$ of $\Gamma([25])$. In general, we have the natural embedding $L(\Gamma) \cong\left\{u_{\gamma} \mid \gamma \in \Gamma\right\}^{\prime \prime} \subset B \rtimes_{\sigma} \Gamma$.

Secondly, if $B=L^{\infty}(X)$, for some standard probability space $(X, \mu)$, then $\sigma$ comes from a probability measure preserving (p.m.p.) action $\Gamma \curvearrowright^{\sigma}(X, \mu)$. The crossed product algebra $L^{\infty}(X) \rtimes_{\sigma} \Gamma$ is called the group measure space construction associated with $\sigma([24])$. If $\Gamma$ is infinite and $\sigma$ is (essentially) free and ergodic, then $L^{\infty}(X) \rtimes_{\sigma} \Gamma$ is a $\mathrm{II}_{1}$ factor and $L^{\infty}(X)$ is a Cartan subalgebra; i.e., it is regular and maximal abelian.

Two free, ergodic p.m.p. actions $\Gamma \curvearrowright(X, \mu)$ and $\Lambda \curvearrowright(Y, \nu)$ are said to be - conjugate if there exist a measure space isomorphism $\theta: X \rightarrow Y$ and a group isomorphism $\delta: \Gamma \rightarrow \Lambda$ such that $\theta(\gamma x)=\delta(\gamma) \theta(x)$, for almost every $x \in X$,

- orbit equivalent if there exists a measure space isomorphism $\theta: X \rightarrow Y$ such that $\theta(\Gamma x)=\Lambda \theta(x)$, for almost every $x \in X$, and

- $\mathrm{W}^{*}$-equivalent (or von Neumann equivalent) if $L^{\infty}(X) \rtimes \Gamma \cong L^{\infty}(Y) \rtimes \Lambda$.

If two actions are conjugate, then they are orbit equivalent. In turn, two actions are orbit equivalent if and only if the inclusions $\left(L^{\infty}(X) \subset L^{\infty}(X) \rtimes \Gamma\right)$ and $\left(L^{\infty}(Y) \subset L^{\infty}(Y) \rtimes \Lambda\right)$ are isomorphic (43], 13] $)$. This shows that orbit equivalent actions are $\mathrm{W}^{*}$-equivalent; the converse is false by 9 .

A p.m.p. action $\Gamma \curvearrowright(X, \mu)$ is weakly mixing if for any measurable sets $A_{1}, \ldots, A_{n}$ of $X$ and every $\varepsilon>0$ we can find $\gamma \in \Gamma$ such that $\left|\mu\left(\gamma A_{i} \cap A_{j}\right)-\mu\left(A_{i}\right) \mu\left(A_{j}\right)\right|<\varepsilon$, for all $i, j$. It is mixing if for any measurable sets $A_{1}, A_{2} \subset X$ and every $\varepsilon>0$ we can find $F \subset \Gamma$ finite such that $\left|\mu\left(\gamma A_{i} \cap A_{j}\right)-\mu\left(A_{i}\right) \mu\left(A_{j}\right)\right|<\varepsilon$, for all $\gamma \in \Gamma \backslash F$.

1.3. Popa's intertwining technique. In 33, S. Popa introduced a very powerful technique for proving unitary conjugacy of two subalgebras of a finite von Neumann algebra. Throughout the paper, this technique will play a central role. Here, we recall Popa's result and establish a strengthening of a particular case of it that will be crucial in the proof of our main results.

Theorem 1.3.1 (see 33, Theorem 2.1, and Corollary 2.3). Let $(M, \tau)$ be a finite von Neumann algebra together with two, possibly non-unital, von Neumann subalgebras $B$ and $Q$ (with units $1_{B}$ and $1_{Q}$, respectively). 
Then the following are equivalent:

(1) There exist non-zero projections $q \in Q, p \in B, a *$-homomorphism $\psi: q Q q \rightarrow$ $p B p$ and a non-zero partial isometry $v \in p M q$ such that $\psi(x) v=v x$, for all $x \in q Q q$.

(2) There exist $a_{1}, \ldots, a_{n} \in 1_{B} M 1_{Q}$ and $\varepsilon>0$ such that $\sum_{i, j=1}^{n}\left\|E_{B}\left(a_{i} u a_{j}^{*}\right)\right\|_{2}^{2} \geq \varepsilon$, for all $u \in \mathcal{U}(Q)$.

(3) There exist $a_{1}, \ldots, a_{n} \in 1_{B} M 1_{Q}, \varepsilon>0$ and a group $\mathcal{U} \subset \mathcal{U}(Q)$ such that $\mathcal{U}^{\prime \prime}=Q$ and $\sum_{i, j=1}^{n}\left\|E_{B}\left(a_{i} u a_{j}^{*}\right)\right\|_{2}^{2} \geq \varepsilon$, for all $u \in \mathcal{U}$.

If one of these conditions holds true, we say that a corner of $Q$ embeds into $B$ inside $M$ and write $Q \prec_{M} B$. Note that if $B_{1}, B_{2}, \ldots$ is a sequence of von Neumann subalgebras of $M$ such that $Q \nprec_{M} B_{i}$, for all $i \geq 1$, then for every group $\mathcal{U} \subset \mathcal{U}(Q)$ with $\mathcal{U}^{\prime \prime}=Q$ we can find a sequence $\left\{u_{n}\right\}_{n \geq 1} \subset \mathcal{U}$ such that $\lim _{n \rightarrow \infty}\left\|E_{B_{i}}\left(a u_{n} b\right)\right\|_{2}=0$, for all $i \geq 1$ and every $a, b \in M$. This statement follows from Theorem 1.3.1 (see [17, Proof of Theorem 4.3 or [45], Remark 3.3).

If $M=B \rtimes \Gamma$, for some action of a countable group $\Gamma$, then condition (2) is equivalent to the following: there exist $F \subset \Gamma$ finite and $\varepsilon>0$ such that for every $u \in U(Q)$, the Fourier coefficients $b_{\gamma}=E_{B}\left(u u_{\gamma}^{*}\right)$ satisfy $\max _{\gamma \in F}\left\|b_{\gamma}\right\|_{2} \geq$ $\varepsilon$. Below, we show that this is equivalent with the apparently weaker condition $\max _{\gamma \in \Gamma}\left\|b_{\gamma}\right\|_{2} \geq \varepsilon$, for all $u \in \mathcal{U}(Q)$.

Theorem 1.3.2. Let $\sigma: \Gamma \rightarrow \operatorname{Aut}(B)$ be an action of a countable group $\Gamma$ on a finite von Neumann algebra $(B, \tau)$ and set $M=B \rtimes_{\sigma} \Gamma$. Let $Q \subset M$ be a von Neumann subalgebra. If there exists $\varepsilon>0$ such that $\max _{\gamma \in \Gamma}\left\|E_{B}\left(u u_{\gamma}^{*}\right)\right\|_{2} \geq \varepsilon$, for all $u \in \mathcal{U}(Q)$, then $Q \prec_{B} M$.

Proof. We begin the proof with the following:

Claim 1. Let $\alpha$ be the flip automorphism of $Q \bar{\otimes} Q$, i.e. $\alpha(x \otimes y)=y \otimes x$, for all $x, y \in Q$, and let $\mathcal{Q}=(Q \bar{\otimes} Q)^{\alpha}$ be the von Neumann algebra of $\alpha$-fixed points. Then $\mathcal{U}:=\{u \otimes u \mid u \in \mathcal{U}(Q)\} \subset \mathcal{U}(\mathcal{Q})$ satisfies $\mathcal{U}^{\prime \prime}=\mathcal{Q}$.

Proof of Claim 1. Let $\mathcal{H}$ be the $\|.\|_{2}$-closure of the span of $\mathcal{U}$. Then the claim is equivalent to $L^{2}(\mathcal{Q})=\mathcal{H}$.

First notice that the span of $\{x \otimes y+y \otimes x \mid x, y \in Q\}$ is dense in $L^{2}(\mathcal{Q})$. Since $x, y \in Q$ are finite linear combinations of Hermitian elements of $Q$, the span of $\left\{h_{1} \otimes h_{2}+h_{2} \otimes h_{1} \mid h_{1}=h_{1}^{*} \in Q, h_{2}=h_{2}^{*} \in Q\right\}$ is also dense in $L^{2}(\mathcal{Q})$. Next, remark that for every $h_{1}, h_{2} \in Q$ we have that $h_{1} \otimes h_{2}+h_{2} \otimes h_{1}=\left(h_{1}+h_{2}\right) \otimes\left(h_{1}+h_{2}\right)-$ $h_{1} \otimes h_{1}-h_{2} \otimes h_{2}$.

Altogether, to prove the claim it suffices to show that for every Hermitian element $h \in Q$ we have that $h \otimes h \in \mathcal{H}$. Towards proving this, fix $h=h^{*} \in Q$. Let $P$ be the orthogonal projection from $L^{2}(\mathcal{Q})$ onto $\mathcal{H}$ and let $a: \mathbb{R} \rightarrow \mathcal{H}$ be given by $a(t)=P\left(e^{i t h} \otimes e^{i t h}\right)$. By the definition of $\mathcal{H}$ we get that $a(t)=0$, for all $t \in \mathbb{R}$. On the other hand, we have that $e^{i t h} \otimes e^{i t h}=\sum_{m, n \geq 0} \frac{1}{m ! n !} i^{m+n} t^{m+n}\left(h^{m} \otimes h^{n}\right)$, where the sum is absolutely convergent in the uniform norm and thus in $\|.\|_{2}$. Therefore we have that $0=a(t)=\sum_{m, n>0} \frac{1}{m ! n !} i^{m+n} t^{m+n} P\left(h^{m} \otimes h^{n}\right)$, for all $t \in \mathbb{R}$, and by analyticity all the coefficients of $a$ must be equal to 0 . In particular, we derive that $h \otimes 1+1 \otimes h \in \mathcal{H}$ and $\frac{1}{2}\left(h^{2} \otimes 1\right)+h \otimes h+\frac{1}{2}\left(1 \otimes h^{2}\right) \in \mathcal{H}$, for all Hermitian $h \in Q$. Thus $h \otimes h \in \mathcal{H}$, which concludes the proof of the claim.

Further, we can assume that $Q$ is diffuse; otherwise the conclusion of the lemma is trivial. Then we can find $v \in \mathcal{U}(Q \bar{\otimes} Q)$ such that $\alpha(v)=-v$. Indeed, $Q$ contains 
a copy of $L^{\infty}([0,1])$, so we can just take $v \in L^{\infty}([0,1] \times[0,1]) \subset Q \bar{\otimes} Q$ given by $v(x, y)=1$ if $x \geq y$ and by $v(x, y)=-1$ if $x<y$. If we let $\mathcal{V}=\mathcal{U}(\mathcal{Q}) \cup \mathcal{U}(\mathcal{Q}) v$, then $\mathcal{V}$ is a subgroup of $\mathcal{U}(Q \bar{\otimes} Q)$ and $\mathcal{V}^{\prime \prime}=Q \bar{\otimes} Q$.

Now, write $M \bar{\otimes} M=(B \bar{\otimes} B) \rtimes(\Gamma \times \Gamma)$, let $\Delta(\Gamma)=\{(\gamma, \gamma) \mid \gamma \in \Gamma\} \subset \Gamma \times \Gamma$ and set $N=(B \bar{\otimes} B) \rtimes \Delta(\Gamma)$.

Claim 2. $Q \bar{\otimes} Q \prec_{M \bar{\otimes} M} N$.

Proof of Claim 2. We start by noticing that for every $u \in \mathcal{U}(Q)$,

$$
\begin{gathered}
\left\|E_{N}(u \otimes u)\right\|_{2}^{2}=\left\|E_{N}\left(\sum_{\left(\gamma_{1}, \gamma_{2}\right) \in \Gamma \times \Gamma}\left(E_{B}\left(u u_{\gamma_{1}}^{*}\right) \otimes E_{B}\left(u u_{\gamma_{2}}^{*}\right)\right) u_{\left(\gamma_{1}, \gamma_{2}\right)}\right)\right\|_{2}^{2} \\
\left.=\| \sum_{\gamma \in \Gamma}\left(E_{B}\left(u u_{\gamma}^{*}\right) \otimes E_{B}\left(u u_{\gamma}^{*}\right)\right) u_{(\gamma, \gamma)}\right)\left\|_{2}^{2}=\sum_{\gamma \in \Gamma}\right\| E_{B}\left(u u_{\gamma}^{*}\right) \|_{2}^{4} \geq \varepsilon^{4} .
\end{gathered}
$$

By combining Claim 1 with Popa's result (Theorem 1.3.1) we get that $\mathcal{Q} \prec_{M \bar{\otimes}} M$ $N$. Since $\mathcal{V}=\mathcal{U}(\mathcal{Q}) \cup \mathcal{U}(\mathcal{Q}) v$ is a group and satisfies $\mathcal{V}^{\prime \prime}=Q \bar{\otimes} Q$, it easily follows that $Q \bar{\otimes} Q \prec_{M \bar{\otimes} M} N$, as claimed.

We are now ready to show that $Q \prec_{M} B$. By Claim 2, we can find $a_{1}, \ldots, a_{n} \in$ $M \bar{\otimes} M$ and $c>0$ such that $\sum_{i, j=1}^{n}\left\|E_{N}\left(a_{i} u a_{j}^{*}\right)\right\|_{2}^{2} \geq c$, for all $u \in \mathcal{U}(Q \bar{\otimes} Q)$. By using $\|.\|_{2}$ approximations (see Section 1.2) and the fact that $E_{N}$ is $N$-bimodular, we may assume that $a_{i}=u_{\left(e, \gamma_{i}\right)}$, for some $\gamma_{i} \in \Gamma$. Then, for every $u \in \mathcal{U}(Q)$, $u \otimes 1 \in \mathcal{U}(Q \bar{\otimes} Q)$; hence

$$
c \leq \sum_{i, j}^{n} \| E_{N}\left(a_{i}(u \otimes 1) a_{j}^{*}\left\|_{2}^{2}=\sum_{i, j=1}^{n}\right\| E_{N}\left(u \otimes u_{\gamma_{i} \gamma_{j}^{-1}}\right)\left\|_{2}^{2}=\sum_{i, j=1}^{n}\right\| E_{B}\left(u u_{\gamma_{i} \gamma_{j}^{-1}}^{*}\right) \|_{2}^{2},\right.
$$

which proves the lemma.

We end this subsection with the following lemma, whose proof we leave as an exercise.

Lemma 1.3.3. Let $(M, \tau)$ be a finite von Neumann algebra and let $Q, B \subset M$ be two von Neumann subalgebras. Let $p \in \mathcal{Z}(Q)$ be a central projection and set $p^{\prime}=\bigvee_{u \in \mathcal{N}_{M}(Q)} u_{p u}^{*} \in \mathcal{Z}(Q)$. If $Q p^{\prime} \prec_{M} B$, then $Q p \prec_{M} B$.

1.4. Rigid inclusions of von Neumann algebras. We next recall S. Popa's notion of rigidity for inclusions of finite von Neumann algebras. Let $(M, \tau)$ be a finite von Neumann algebra together with a von Neumann subalgebra $B$.

The inclusion $(B \subset M)$ is rigid (or, has the relative property $(T)$ ) if any sequence of subunital $\left(\Phi_{n}(1) \leq 1\right)$, subtracial $\left(\Phi_{n} \circ \tau \leq \tau\right)$, normal completely positive maps $\Phi_{n}: M \rightarrow M$ which satisfy $\lim _{n \rightarrow \infty}\left\|\Phi_{n}(x)-x\right\|_{2}=0$, for all $x \in M$, converges to the identity uniformly on the unit ball of $B$, i.e. $\lim _{n \rightarrow \infty} \sup _{x \in B,\|x\| \leq 1} \| \Phi_{n}(x)-$ $x \|_{2}=0$ ([35]). In the case when $B=M$, we say that $M$ has property (T) ([10]).

For two countable groups $\Gamma_{0} \subset \Gamma$, the inclusion $\left(L\left(\Gamma_{0}\right) \subset L(\Gamma)\right)$ is rigid if and only if the inclusion $\left(\Gamma_{0} \subset \Gamma\right)$ has the relative property $(T)$ ([35, Proposition 5.1). By the classical results of Kazhdan and Margulis, the inclusions $\left(\mathrm{SL}_{n}(\mathbb{Z}) \subset \mathrm{SL}_{n}(\mathbb{Z})\right)$ $(n \geq 3)$ and $\left(\mathbb{Z}^{2} \subset \mathbb{Z}^{2} \rtimes \mathrm{SL}_{2}(\mathbb{Z})\right)$ have the relative property $\left.(\mathrm{T})([20], 23]\right)$. Recall that a group $\Gamma$ has property $(\mathrm{T})$ of Kazhdan if and only if the inclusion $(\Gamma \subset \Gamma)$ has the relative property $(\mathrm{T})$. For examples of property $(\mathrm{T})$ groups, see the extensive monograph [1]. 
1.5. Weakly malleable deformations of Bernoulli actions. S. Popa discovered that Bernoulli actions $\Gamma \curvearrowright\left(X_{0}, \mu_{0}\right)^{\Gamma}$ have a remarkable deformation property, and called it malleability ([33]). By pairing it with property $(\mathrm{T})$ of the group $\Gamma$, he proved striking rigidity results concerning the associated $\mathrm{II}_{1}$ factor ([33], 34]).

Since then, malleable deformations have been found in several other contexts and are now a central tool in Popa's deformation/rigidity theory (see [38, Section 6). In [14, in order to extend some of Popa's results [33, 34 to $\mathrm{II}_{1}$ factors coming from Bernoulli actions with an arbitrary base, we introduced a new class of malleable deformations.

To recall their construction from [14, Section 2, let $(B, \tau)$ be a finite von Neumann algebra and let $\Gamma \curvearrowright I$ be an action of a countable group on a countable set. For every set $J \subset I$, we denote $B^{J}=\bar{\bigotimes}_{i \in J}(B)_{i}$. Whenever $J \subset J^{\prime}$, we view $B^{J}$ as a subalgebra of $B^{J^{\prime}}$, in the natural way. Let $\sigma: \Gamma \rightarrow \operatorname{Aut}\left(B^{I}\right)$ be the generalized Bernoulli action defined by $\sigma(\gamma)\left(\bigotimes_{i \in I} x_{i}\right)=\bigotimes_{i \in I} x_{\gamma^{-1} \cdot i}$, for every $x=\bigotimes_{i \in I} x_{i} \in B^{I}$ and $\gamma \in \Gamma$. Let $M=B^{I} \rtimes_{\sigma} \Gamma$ be the associated crossed product von Neumann algebra.

Next, we augment $M$ to a von Neumann algebra $\tilde{M}$ and define a 1-parameter group of automorphisms $\left\{\theta_{t}\right\}_{t \in \mathbb{R}}$ of $\tilde{M}$ such that $\theta_{t} \rightarrow \operatorname{id}_{\tilde{M}}$, in the pointwise $\|.\|_{2}$ topology, as $t \rightarrow 0$. Towards this, we define the free product von Neumann algebra $\tilde{B}=B * L(\mathbb{Z})$. Let $\tilde{\sigma}: \Gamma \rightarrow \operatorname{Aut}\left(\tilde{B}^{I}\right)$ be the generalized Bernoulli action. It is clear that $B^{I} \subset \tilde{B}^{I}$ and that $\tilde{\sigma}$ extends $\sigma$; hence we have the inclusion $M \subset \tilde{M}:=$ $\tilde{B}^{I} \rtimes_{\tilde{\sigma}} \Gamma$.

Now, let $u \in L(\mathbb{Z})$ be a Haar unitary such that $L(\mathbb{Z})=\left\{u^{n} \mid n \in \mathbb{Z}\right\}^{\prime \prime}$ and let $h \in L(\mathbb{Z})$ be a Hermitian element such that $u=e^{i h}$. For every $t \in \mathbb{R}$, we define the unitary element $u_{t}=e^{i t h} \in L(\mathbb{Z})$ and consider the automorphism $\theta_{t}=$ $\bigotimes_{i \in I} \operatorname{Ad}\left(u_{t}\right)_{i}$ of $\tilde{B}^{I}$. Since $\theta_{t}$ commutes with $\tilde{\sigma}$, it extends to an automorphism of $\tilde{M}$ through the formula $\theta_{t}(x)=\sum_{\gamma \in \Gamma} \theta_{t}\left(x_{\gamma}\right) u_{\gamma}$, for all $x=\sum_{\gamma \in \Gamma} x_{\gamma} u_{\gamma} \in \tilde{M}$. Since $\lim _{t \rightarrow 0}\left\|u_{t}-1\right\|_{2}=0$, we get that $\theta_{t} \rightarrow \operatorname{id}_{\tilde{M}}$ as $t \rightarrow 0$.

We end this section by noticing that $\left\{\theta_{t}\right\}_{t \in \mathbb{R}}$ admits a certain $\beta$-symmetry (see e.g. [33, Section 1.4).

Lemma 1.5.1. There exists an automorphism $\beta$ of $\tilde{M}$ such that $\beta^{2}=i d_{\tilde{M}}, \beta_{\mid M}=$ $i d_{M}$ and $\beta \theta_{t} \beta=\theta_{-t}$, for all $t \in \mathbb{R}$.

Proof. Since $u$ generates $L(\mathbb{Z})$, it follows that $M$ and $\left\{\bigotimes_{i \in I} u^{n_{i}}\left|n_{i} \in \mathbb{Z},\right|\{i \in\right.$ $\left.\left.I \mid n_{i} \neq 0\right\} \mid<\infty\right\}$ generate $\tilde{M}$ as a von Neumann algebra. Using this observation it is immediate to see that $\beta$ defined by $\beta_{\mid M}=\operatorname{id}_{M}$ and $\beta\left(\bigotimes_{i \in I} u^{n_{i}}\right)=\bigotimes_{i \in I} u^{-n_{i}}$, for every $n_{i} \in \mathbb{Z}$ such that $\left\{i \in I \mid n_{i} \neq 0\right\}$ is finite, extends to an automorphism of $\tilde{M}$ with the desired properties.

\section{Rigid SUbALGEBRAS OF II $_{1}$ FACTORS ARISING FROM GENERALIZED BERNOULLI ACTIONS}

Let $M=B^{\Gamma} \rtimes \Gamma$ be the $\mathrm{II}_{1}$ factor associated with a Bernoulli action. S. Popa showed, as part of his deformation/rigidity theory, that if $B$ is abelian, then any rigid subalgebra $Q$ of $M$ whose normalizer generates a factor can be conjugated inside $L(\Gamma)$ by a unitary element ([33], Theorem 4.1). The proof exploits the tension between the rigidity of the inclusion $Q \subset M$ and the malleability property of Bernoulli actions. 
In [14, Theorems 3.6, 3.7, by using the weakly malleable deformations associated with $M$ we showed that any rigid subalgebra $Q$ of $M$ has a corner which embeds into $L(\Gamma)$, regardless of any regularity property of $Q$. In this section, by relying on ideas and techniques from [14] and by following a deformation/rigidity strategy, we extend the last result to the class of generalized Bernoulli actions.

Theorem 2.1. Let $\Gamma$ a countable group acting on a countable set $I$. For $i \in I$, denote by $\Gamma_{i}$ its stabilizer in $\Gamma$. Let $(B, \tau)$ be an abelian von Neumann algebra. Let $\sigma: \Gamma \rightarrow \operatorname{Aut}\left(B^{I}\right)$ be the generalized Bernoulli action and denote $M=B^{I} \rtimes_{\sigma} \Gamma$. Suppose that $Q$ is a von Neumann subalgebra of $M$ such that the inclusion $(Q \subset M)$ is rigid.

Then $Q \prec_{M} L(\Gamma)$. Moreover, if $\Gamma$ is $I C C$ and $Q \nprec_{M} L\left(\Gamma_{i}\right)$, for every $i \in I$, then there exists a unitary $u \in M$ such that $u Q u^{*} \subset L(\Gamma)$. Furthermore, in this case, we have that $u P u^{*} \subset L(\Gamma)$, where $P$ is the von Neumann algebra generated by the quasi-normalizer of $Q$ in $M$.

This theorem generalizes three other results in the literature. The first part and the moreover part of Theorem 2.1 extend respectively 45], Lemma 5.2 and [40], Theorem 6.5 in whose hypotheses it is additionally assumed that $P \nprec_{M} B^{I} \rtimes_{\sigma} \Gamma_{i}$, for all $i \in I$. Theorem 2.1 also generalizes (a particular case of) [16], Proposition 3.3, which shows that $M$ admits no rigid subalgebra $Q$ such that $Q \subset B^{I}$.

Proof. Let $\tilde{M}$ and $\left\{\theta_{t}\right\}_{t \in \mathbb{R}}$ be defined as in Section 1.5.

Since the inclusion $Q \subset M$ is rigid, the inclusion $Q \subset \tilde{M}$ is rigid (by [35], Proposition 4.6). Since $\theta_{t} \rightarrow \mathrm{id}_{\tilde{M}}$, we can find $t>0$ such that $\left\|\theta_{t}(u)-u\right\|_{2} \leq \frac{1}{2}$, for every $u \in \mathcal{U}(Q)$.

Let $v$ be the unique element of minimal $2-$ norm in the $\|\cdot\|_{2}-$ closed convex hull of the set $\left\{\theta_{t}(u) u^{*} \mid u \in \mathcal{U}(Q)\right\}$. Then $\|v-1\|_{2} \leq \frac{1}{2}$; hence $v \neq 0$. Also, by construction, $v \in \tilde{M},\|v\| \leq 1$ and $\theta_{t}(u) v=v u$, for all $u \in \mathcal{U}(Q)$.

Next, let $\varepsilon>0$ such that $\delta:=\|v\|_{2}-\left(1+\frac{2}{\sqrt{1-\left|\tau\left(u_{t}\right)\right|^{4}}}\right) \varepsilon>0$. Then we can find finite sets $F \subset I, K \subset \Gamma$ and $w$ in the linear span of $\left\{\tilde{B}^{F} u_{\gamma} \mid \gamma \in K\right\}$ such that $\|w-v\|_{2} \leq \varepsilon$ and $\|w\| \leq 1$. Suppose for simplicity that $K=\left\{\gamma^{-1} \mid \gamma \in K\right\}$. Also, we use the notation $K F=\{k \cdot i \mid k \in K, i \in F\}$. Denote by $T$ the orthogonal projection from $L^{2}(\tilde{M})$ onto the closed linear span of $\left\{\left(\tilde{B}^{F} \otimes B^{\gamma K F \backslash F}\right) u_{\gamma} \mid \gamma \in \Gamma\right\}$.

Claim 1. For all $u \in \mathcal{U}(Q)$, we have $\|T(w u)\|_{2} \geq \delta$.

Proof of Claim 1. Fix $u \in \mathcal{U}(Q)$. Then $\left\|\theta_{t}(u) w-w u\right\|_{2}=\| \theta_{t}(u)(w-v)-(w-$ $v) u \|_{2} \leq 2 \varepsilon$. Decompose $w u=\sum_{\gamma \in \Gamma} a_{\gamma} u_{\gamma}$ and $\theta_{t}(u) w=\sum_{\gamma \in \Gamma} b_{\gamma} u_{\gamma}$.

Since $u \in M, w \in \sum_{\gamma \in K} \tilde{B}^{F} u_{\gamma}$ and $K=K^{-1}$, an easy calculation shows that $a_{\gamma} \in \tilde{B}^{F \bar{\otimes}} B^{I \backslash F}$ and $b_{\gamma} \in \tilde{B}^{\gamma K F} \bar{\otimes}\left(u_{t} B u_{t}^{*}\right)^{I \backslash \gamma K F}$, for all $\gamma \in \Gamma$.

Denote by $S_{\gamma}$ and $R_{\gamma}$ the orthogonal projections from $L^{2}\left(\tilde{B}^{I}\right)$ onto $L^{2}\left(\tilde{B}^{F \cup \gamma K F}\right)$ and onto $L^{2}\left(\tilde{B}^{\gamma K F} \bar{\otimes}\left(u_{t} B u_{t}^{*}\right)^{I \backslash \gamma K F}\right)$, respectively. Since $a_{\gamma} \in \tilde{B}^{F} \bar{\otimes} B^{I \backslash F}$, [16], Lemma 3.5 implies that

$$
\left\|R_{\gamma}\left(a_{\gamma}\right)\right\|_{2}^{2} \leq\left|\tau\left(u_{t}\right)\right|^{4}\left\|a_{\gamma}\right\|_{2}^{2}+\left(1-\left|\tau\left(u_{t}\right)\right|^{4}\right)\left\|S_{\gamma}\left(a_{\gamma}\right)\right\|_{2}^{2}, \forall \gamma \in \Gamma .
$$

Now, since $R_{\gamma}\left(b_{\gamma}\right)=b_{\gamma}$, for every $\gamma \in \Gamma$, we get that

$$
\sum_{\gamma \in \Gamma}\left\|a_{\gamma}-R_{\gamma}\left(a_{\gamma}\right)\right\|_{2}^{2} \leq \sum_{\gamma \in \Gamma}\left\|a_{\gamma}-b_{\gamma}\right\|_{2}^{2}=\left\|w u-\theta_{t}(u) w\right\|_{2}^{2} \leq 4 \varepsilon^{2} .
$$


On the other hand, (2.a) implies that

$$
\sum_{\gamma \in \Gamma}\left\|a_{\gamma}-R_{\gamma}\left(a_{\gamma}\right)\right\|_{2}^{2} \geq\left(1-\left|\tau\left(u_{t}\right)\right|^{4}\right) \sum_{\gamma \in \Gamma}\left\|a_{\gamma}-S_{\gamma}\left(a_{\gamma}\right)\right\|_{2}^{2} .
$$

Since $a_{\gamma} \in \tilde{B}^{F} \bar{\otimes} B^{I \backslash F}$ we have $T(w u)=\sum_{\gamma \in \Gamma} S_{\gamma}\left(a_{\gamma}\right) u_{\gamma}$. By combining (2.b) and (2.c) we get that $\|T(w u)-w u\|_{2}^{2} \leq 4 \varepsilon^{2}\left(1-\left|\tau\left(u_{t}\right)\right|^{4}\right)^{-1}$, which yields the claim.

Next, let $\left\{B_{n}\right\}_{n \geq 1}$ be an increasing sequence of unital, finite dimensional subalgebras of $B$ such that $\bigcup_{n \geq 1} B_{n}$ is dense in $B$. For $n \geq 1$, set $M_{n}=B_{n}^{I} \rtimes \Gamma$ and denote by $E_{n}$ the conditional expectation from $M$ onto $M_{n}$. Since the inclusion $Q \subset M$ is rigid and $E_{n} \rightarrow \operatorname{id}_{M}$, we can find $n_{0} \geq 1$ such that $\left\|E_{n_{0}}(u)-u\right\|_{2} \leq \frac{\delta}{2}$, for all $u \in \mathcal{U}(Q)$. Together with (2.c) this yields

$$
\left\|T\left(w E_{n_{0}}(u)\right)\right\|_{2} \geq \frac{\delta}{2}, \forall u \in \mathcal{U}(Q) .
$$

Claim 2. We have $Q \prec_{M} L(\Gamma)$.

Proof of Claim 2. Assuming this is false, we get a sequence $\left\{u_{m}\right\}_{m \geq 1} \subset \mathcal{U}(Q)$ such that $\left\|E_{L(\Gamma)}\left(a u_{m} b\right)\right\|_{2} \rightarrow 0$, for all $a, b \in M$. We will prove that

$$
\left\|T\left(c u_{g} E_{n_{0}}\left(u_{m}\right)\right)\right\|_{2} \rightarrow 0, \forall c \in \tilde{B}^{F}, g \in \Gamma .
$$

Note that since $w$ belongs to the linear span of $\left\{\tilde{B}^{F} u_{\gamma} \mid \gamma \in K\right\}$, (2.e) implies that $\left\|T\left(w E_{n_{0}}\left(u_{m}\right)\right)\right\|_{2} \rightarrow 0$, which contradicts (2.d).

To deduce (2.e), write $u_{m}=\sum_{\gamma \in \Gamma} x_{m}^{\gamma} u_{\gamma}$, where $x_{m}^{\gamma} \in B^{\Gamma}$, and assume $\|c\| \leq 1$. Since $E_{n_{0} \mid B^{\Gamma}}=E_{B_{n_{0}}^{\Gamma}}$ and $\sigma$ commute we have

$$
c u_{g} E_{n_{0}}\left(u_{m}\right)=\sum_{\gamma \in \Gamma} c E_{B_{n_{0}}^{\Gamma}}\left(\sigma_{g}\left(x_{m}^{\gamma}\right)\right) u_{g \gamma} .
$$

As $c \in \tilde{B}^{F}$, we get that

$$
\begin{aligned}
T\left(c u_{g} E_{n_{0}}\left(u_{m}\right)\right) & =\sum_{\gamma \in \Gamma} E_{\tilde{B}^{F} \bar{\otimes} B^{g \gamma K F \backslash F}}\left(c E_{B_{n_{0}}^{\Gamma}}\left(\sigma_{g}\left(x_{m}^{\gamma}\right)\right) u_{g \gamma}\right. \\
& =c \sum_{\gamma \in \Gamma} E_{B_{n_{0}}^{F \cup g \gamma K F}}\left(\sigma_{g}\left(x_{m}^{\gamma}\right)\right) u_{g \gamma} .
\end{aligned}
$$

Thus, as $\|c\| \leq 1$, we have

$$
\left\|T\left(c u_{g} E\left(u_{m}\right)\right)\right\|_{2}^{2} \leq \sum_{\gamma \in \Gamma}\left\|E_{B_{n_{0}}^{F \cup g \gamma K F}}\left(\sigma_{g}\left(x_{m}^{\gamma}\right)\right)\right\|_{2}^{2}=\sum_{\gamma \in \Gamma}\left\|E_{B_{n_{0}}^{g-1} F \cup \gamma K F}\left(x_{m}^{\gamma}\right)\right\|_{2}^{2} .
$$

Let $\left\{\xi_{i}\right\}_{i=1}^{l}$ be an orthonormal basis for $B_{n_{0}}$. For $J \subset I$ finite and $s=\left(s_{i}\right)_{i \in J} \in$ $\{1, \ldots, l\}^{J}$, set $\xi_{s}=\bigotimes_{i \in J} \xi_{s_{i}} \in B_{n_{0}}^{J}$. Then $\left\{\xi_{s}\right\}_{s \in\{1, \ldots, l\}^{J}}$ is an orthonormal basis for $B_{n_{0}}^{J}$; hence

$$
\begin{gathered}
\left\|E_{B_{n_{0}}^{g-1} F \cup \gamma K F}\left(x_{m}^{\gamma}\right)\right\|_{2}^{2}=\sum_{s \in\{1, . ., l\}^{g^{-1}}} \sum_{F} \sum_{t \in\{1, . .,\}^{\gamma K F \backslash g^{-1} F}}\left|\tau\left(\xi_{s} x_{m}^{\gamma} \xi_{t}\right)\right|^{2} \\
\leq \sum_{s \in\{1, . ., l\}^{g^{-1}}} \sum_{t \in\{1, . ., l\}^{K F}}\left|\tau\left(\xi_{s} x_{m}^{\gamma} \sigma_{\gamma}\left(\xi_{t}\right)\right)\right|^{2} .
\end{gathered}
$$


The combination of (2.f) and (2.g) gives that

$$
\left\|T\left(c u_{g} E\left(u_{m}\right)\right)\right\|_{2}^{2} \leq \sum_{s \in\{1, . ., l\}^{g^{-1}}} \sum_{t \in\{1, . ., l\}^{K F}}\left\|E_{L(\Gamma)}\left(\xi_{s} u_{m} \xi_{t}\right)\right\|_{2}^{2} \rightarrow 0 .
$$

For the moreover part of the statement, assume that $\Gamma$ is ICC, i.e. $L(\Gamma)$ is a factor, and that $Q \nprec_{M} L\left(\Gamma_{i}\right)$, for all $i \in I$.

Claim 3. For every relatively rigid von Neumann subalgebra $Q \subset M$ which satisfies $Q \nprec_{M} L\left(\Gamma_{i}\right)$, for all $i \in I$, we can find a unitary $u \in M$ and a non-zero projection $q^{\prime} \in Q^{\prime} \cap M$ such that $u\left(Q q^{\prime}\right) u^{*} \subset L(\Gamma)$.

Proof of Claim 3. By the first part of the proof we have that $Q \prec_{M} L(\Gamma)$. Since $Q \nprec_{M} L\left(\Gamma_{i}\right)$, by [45], Remark 3.8 we can find non-zero projections $q \in Q, p \in L(\Gamma)$, a $*$-homomorphism $\psi: q Q q \rightarrow p L(\Gamma) p$ and a partial isometry $0 \neq v \in p M q$ such that $\psi(x) v=v x$, for all $x \in q Q q$, and $\psi(q Q q) \nprec_{L(\Gamma)} L\left(\Gamma_{i}\right)$, for all $i \in I$. Thus, by 45, Lemma 4.2(1) we deduce that $\psi(q Q q)^{\prime} \cap p L(\Gamma) p \subset p L(\Gamma) p$. In particular, it follows that $v v^{*} \in p L(\Gamma) p$ and hence $v Q v^{*} \subset L(\Gamma)$. Since $v^{*} v \in(q Q q)^{\prime} \cap q M q$ we can find a projection $q^{\prime \prime} \in Q^{\prime} \cap M$ such that $v^{*} v=q q^{\prime \prime}$. Let $w \in M$ be a unitary extending $v$. Then $w\left(q Q q q^{\prime \prime}\right) w^{*}=v Q v^{*} \subset L(\Gamma)$. Since $L(\Gamma)$ is a factor it follows that we can find a unitary $u \in M$ such that $u\left(\left(Q q^{\prime \prime}\right) z\right) u^{*} \subset L(\Gamma)$, where $z$ is the central support of $q q^{\prime \prime}$ in $Q q^{\prime \prime}$. Since the center of $Q q^{\prime \prime}$ is contained in $Q^{\prime} \cap M$ we deduce that $0 \neq q^{\prime}=q^{\prime \prime} z \in \mathcal{P}\left(Q^{\prime} \cap M\right)$ satisfies the claim.

Now, let $q \in Q, q^{\prime} \in Q^{\prime} \cap M$ such that $\tau\left(q q^{\prime}\right)=\frac{1}{n}$, where $n \geq 1$ is an integer, and consider the natural embedding of $Q_{0}:=M_{n \times n}\left(q Q q q^{\prime}\right)$ into $M$. By [35, the inclusion $Q_{0} \subset M$ is rigid. Since $Q \nprec_{M} L\left(\Gamma_{i}\right)$, we get that $Q_{0} \nprec_{M} L\left(\Gamma_{i}\right)$, for all $i \in I$. Thus the conclusion of Claim 3 holds true for $Q_{0}$.

A close inspection of the last part of the proof of [33, Theorem 4.4(ii) reveals that this fact and the factoriality of $L(\Gamma)$ are enough to imply (via a maximality argument) that we can find $u \in \mathcal{U}(M)$ such that $u Q u^{*} \subset L(\Gamma)$. Finally, [45, Lemma 4.2(1) implies that $u P u^{*} \subset L(\Gamma)$.

Remark 2.2. Let $N$ be a finite von Neumann algebra. The above proof can be modified to show that if a Neumann subalgebra $Q \subset M \bar{\otimes} N$ satisfies $\left(\theta_{t} \otimes \operatorname{id}_{N}\right)(x) v=$ $v x$, for every $x \in Q$, for some fixed $0 \neq v \in \tilde{M} \bar{\otimes} N$ and $t \neq 0$, then $Q \prec_{M \bar{\otimes} N}$ $L(\Gamma) \bar{\otimes} N$. To see this, just replace throughout the proof any subalgebra $A \subset M$ with the subalgebra $A \bar{\otimes} N$ of $M \bar{\otimes} N$.

\section{A spectral gap argument}

In the next sections, we prove a series of results concerning tensor products of $\mathrm{II}_{1}$ factors arising from Bernoulli actions (Theorems 3.2, 4.1 and 6.1). These results have natural analogs for a single $\mathrm{II}_{1}$ factor $M=B^{\Gamma} \rtimes \Gamma$ coming from a Bernoulli action with $B$ abelian. For simplicity, we will only describe the latter, although below we state and prove the former. Denote $A=B^{\Gamma}$.

In this section, we prove that if $D \subset M$ is a von Neumann subalgebra such that there exists a sequence $x=\left(x_{n}\right)_{n} \in D^{\prime} \cap M^{\omega}$ on which the deformations $\theta_{t}$ converge uniformly but $x \notin A^{\omega} \rtimes \Gamma$, then a corner of $D$ embeds into $L(\Gamma)$.

This statement was inspired by an idea of J. Peterson. In the context when $\Gamma$ is a free product group and the action of $\Gamma$ on $A$ is compact, he analyzed sequences 
$\left\{x_{n}\right\}_{n} \subset D^{\prime} \cap M$ on which certain deformations converge uniformly in order to derive conjugacy results for $D$ inside $M$ (see [31, Theorem 4.1).

The proof of our result, rather than using arguments from [31, relies on Popa's spectral gap argument (39]).

Before continuing, we fix some notation that we will use throughout the paper.

\section{Notation 3.1.}

- Let $\Gamma_{1}, \Gamma_{2}$ be countable groups and $B_{1}, B_{2}$ be abelian von Neumann algebras.

- Consider the Bernoulli actions $\sigma_{i}: \Gamma_{i} \rightarrow \operatorname{Aut}\left(B_{i}^{\Gamma_{i}}\right)$ and denote $M_{i}=B_{i}^{\Gamma} \rtimes_{\sigma_{i}} \Gamma_{i}$.

- Denote $M=M_{1} \bar{\otimes} M_{2}, A=B_{1}^{\Gamma_{1}} \bar{\otimes} B_{2}^{\Gamma_{2}}$ and $\Gamma=\Gamma_{1} \times \Gamma_{2}$.

- Note that $M=A \rtimes_{\sigma} \Gamma$, where $\sigma\left(\gamma_{1}, \gamma_{2}\right)=\sigma_{1}\left(\gamma_{1}\right) \otimes \sigma_{2}\left(\gamma_{2}\right)$.

Theorem 3.2. Let $\tilde{M}_{i}=\left(B_{i} * L(\mathbb{Z})\right)^{\Gamma} \rtimes \Gamma \supset M_{i}$ and $\left\{\theta_{t}^{i}\right\}_{t \in \mathbb{R}} \subset \operatorname{Aut}\left(\tilde{M}_{i}\right)$ be defined as in Section 1.5. Let $\tilde{M}=\tilde{M}_{1} \bar{\otimes} \tilde{M}_{2}$ and for every $t \in \mathbb{R}$, set $\theta_{t}=\theta_{t}^{1} \otimes \theta_{t}^{2} \in$ $\operatorname{Aut}(\tilde{M})$. Let $D \subset M$ be a von Neumann subalgebra and $x \in D^{\prime} \cap M^{\omega}$ satisfy $\lim _{t \rightarrow 0}\left\|\theta_{t}(x)-x\right\|_{2}=0$. Then one of the following holds true:

(1) $x \in A^{\omega} \rtimes \Gamma$.

(2) $D \prec_{M} M_{1} \bar{\otimes} L\left(\Gamma_{2}\right)$ or $D \prec_{M} L\left(\Gamma_{1}\right) \bar{\otimes} M_{2}$.

Here, we view $\theta_{t}$ as an automorphism of $\tilde{M}^{\omega}$ given by $\theta_{t}\left(\left(x_{n}\right)_{n}\right)=\left(\theta_{t}\left(x_{n}\right)\right)_{n}$. Also, we denote by $A^{\omega} \rtimes \Gamma$ the von Neumann algebra generated by $A^{\omega}$ and $\left\{u_{\gamma}\right\}_{\gamma \in \Gamma}$ inside $M^{\omega}$.

The proof of Theorem 3.2 is based on the following general technical result.

Lemma 3.3. Let $\sigma: \Gamma \rightarrow \operatorname{Aut}(\tilde{A})$ be an action on a finite von Neumann algebra $(\tilde{A}, \tau)$ which leaves invariant a von Neumann subalgebra $A \subset \tilde{A}$. Denote $M=A \rtimes \Gamma$, $\tilde{M}=\tilde{A} \rtimes \Gamma$. Assume that the Hilbert $M$-bimodule $L^{2}(\tilde{M}) \ominus L^{2}(M)$ is isomorphic to $\bigoplus_{i \in I} L^{2}\left(\left\langle M, e_{A_{i}}\right\rangle\right)$, where $A_{i} \subset M$ are von Neumann subalgebras such that for every $i \in I$, we have $A_{i} \subset A \rtimes \Gamma_{i}$, for some finite subgroup $\Gamma_{i} \subset \Gamma$.

Let $\left\{\theta_{t}\right\}_{t \in \mathbb{R}}$ be a 1-parameter group of automorphisms of $\tilde{M}$ and $\beta \in \operatorname{Aut}(\tilde{M})$ such that $\beta^{2}=1_{\tilde{M}}, \beta_{\mid M}=1_{M}, \beta \theta_{t} \beta=\theta_{-t}$, for all $t \in \mathbb{R}$. Let $D \subset M$ be a von Neumann subalgebra. Assume that there exist no $t \neq 0$ and $0 \neq v \in \tilde{M}$ such that $\theta_{t}(y) v=v y$, for all $y \in D$. Then we have

$$
\left\|x-E_{A^{\omega} \rtimes \Gamma}(x)\right\|_{2} \leq 4 \sqrt{2} \liminf _{t \rightarrow 0}\left\|\theta_{t}(x)-x\right\|_{2}, \forall x \in D^{\prime} \cap M^{\omega} .
$$

Proof of Lemma 3.3. Let $x=\left(x_{n}\right)_{n} \in D^{\prime} \cap M^{\omega}$. After rescaling we may assume that $\|x\| \leq 1$. Let $y=x-E_{A^{\omega} \rtimes \Gamma}(x) \in M^{\omega}$. Since $\|y\| \leq 2$, we can represent $y=\left(y_{n}\right)_{n}$, where $y_{n} \in M$ satisfy $\left\|y_{n}\right\| \leq 2$, for all $n$. Assuming the conclusion is false, we can find $0<\varepsilon<\frac{\|y\|_{2}}{4 \sqrt{2}}$ and $t \neq 0$ such that $\left\|\theta_{t}(x)-x\right\|_{2}=\lim _{n \rightarrow \omega} \| \theta_{t}\left(x_{n}\right)-$ $x_{n} \|_{2} \leq \varepsilon$.

For $u \in M$, we denote $\delta_{t}(u):=\theta_{t}(u)-E_{M}\left(\theta_{t}(u)\right) \in L^{2}(\tilde{M}) \ominus L^{2}(M)$.

Claim 1. For every $u \in \mathcal{U}(D)$ we have that $\left\|\left[\delta_{t}(u), y\right]\right\|_{2} \leq 8 \varepsilon$.

Proof of Claim 1. Let $u \in \mathcal{U}(D)$. Since $[u, x]=0$, by using an idea from [39], proof of Theorem 4.1, we have that

$$
\begin{gathered}
\left\|\left[\theta_{t}(u), x\right]\right\|_{2}=\|\left[u, \theta_{-t}(x)\left\|_{2}=\right\|\left[u,\left(\theta_{-t}(x)-x\right)\right] \|_{2}\right. \\
\leq 2\left\|\theta_{-t}(x)-x\right\|_{2}=2\left\|x-\theta_{t}(x)\right\|_{2} \leq 2 \varepsilon .
\end{gathered}
$$


Next, notice that $E_{\tilde{A}^{\omega} \rtimes \Gamma}(x)=\left(E_{\tilde{A}^{\omega} \rtimes \Gamma} \circ E_{M^{\omega}}\right)(x)=E_{A^{\omega} \rtimes \Gamma}(x)$. By combining this and the fact that $\theta_{t}(u) \in \tilde{M} \subset \tilde{A}^{\omega} \rtimes \Gamma$ with (3.a) we get that

$$
\begin{aligned}
\left\|\left[\theta_{t}(u), y\right]\right\|_{2} & =\left\|\left[\theta_{t}(u),\left(x-E_{A^{\omega} \rtimes \Gamma}(x)\right)\right]\right\|_{2} \\
& \leq\left\|\left[\theta_{t}(u), x\right]\right\|_{2}+\left\|\theta_{t}(u) E_{A^{\omega} \rtimes \Gamma}(x)-E_{A^{\omega} \rtimes \Gamma}(x) \theta_{t}(u)\right\|_{2} \\
& \leq 2 \varepsilon+\left\|E_{\tilde{A}^{\omega} \rtimes \Gamma}\left(\theta_{t}(u) x-x \theta_{t}(u)\right)\right\|_{2} \leq 2 \varepsilon+\left\|\theta_{t}(u) x-x \theta_{t}(u)\right\|_{2} \leq 4 \varepsilon .
\end{aligned}
$$

Finally, by using (3.b) we have that

$$
\begin{gathered}
\left\|\left[\delta_{t}(u), y\right]\right\|_{2} \leq 4 \varepsilon+\left\|E_{M}\left(\theta_{t}(u)\right) y-y E_{M}\left(\theta_{t}(u)\right)\right\|_{2} \\
=4 \varepsilon+\left\|E_{M^{\omega}}\left(\theta_{t}(u) y-y \theta_{t}(u)\right)\right\|_{2} \leq 8 \varepsilon
\end{gathered}
$$

and thus the claim is proven.

Claim 2. For every $\xi, \eta \in L^{2}(\tilde{M}) \ominus L^{2}(M)$, we have that $\lim _{n \rightarrow \omega}\left\langle y_{n} \xi, \eta y_{n}\right\rangle=0$.

Proof of Claim 2. Recall that $L^{2}(\tilde{M}) \ominus L^{2}(M)=\bigoplus_{i \in I} L^{2}\left(\left\langle M, e_{A_{i}}\right\rangle\right)$. Since $\left\|y_{n}\right\| \leq$ 2 , for all $n$, it is clearly sufficient to prove the claim for $\xi$ and $\eta$ of the form $\xi=\xi_{1} e_{A_{i}} \xi_{2} \in L^{2}\left(\left\langle M, e_{A_{i}}\right\rangle\right)$ and $\eta=\eta_{1} e_{A_{i}} \eta_{2} \in L^{2}\left(\left\langle M, e_{A_{i}}\right\rangle\right)$, for some $i \in I$, $\xi_{1}, \xi_{2}, \eta_{1}, \eta_{2} \in M$. But, in this case, if $\operatorname{Tr}$ denotes the natural semi-finite trace on $\left\langle M, e_{A_{i}}\right\rangle$, then

$$
\begin{aligned}
\left|\left\langle y_{n} \xi, \eta y_{n}\right\rangle\right| & =\left|\operatorname{Tr}\left(y_{n}^{*} \eta^{*} y_{n} \xi\right)\right|=\mid \operatorname{Tr}\left(y_{n}^{*} \eta_{2}^{*} e_{A_{i}} \eta_{1}^{*} y_{n} \xi_{1} e_{A_{i}} \xi_{2}\right) \| \\
& =\tau\left(E_{A_{i}}\left(\xi_{2} y_{n}^{*} \eta_{2}^{*}\right) E_{A_{i}}\left(\eta_{1}^{*} y_{n} \xi_{1}\right)\right)\left|\leq\left\|E_{A_{i}}\left(\eta_{2} y_{n}^{*} \xi_{2}^{*}\right)\right\|_{2}\right| \mid E_{A_{i}}\left(\eta_{1}^{*} y_{n} \xi_{1}\right) \|_{2} .
\end{aligned}
$$

This reduces the claim to showing that $\lim _{n \rightarrow \omega}\left\|E_{A_{i}}\left(\eta_{1}^{*} y_{n} \xi_{1}\right)\right\|_{2}=0$, for all $i \in I$, $\xi_{1}, \eta_{1} \in M$. To this end, let $\Gamma_{i} \subset \Gamma$ be a finite group such that $A_{i} \subset A \rtimes \Gamma_{i}$. Then, for every $\zeta \in M$ we have that $\left\|E_{A_{i}}(\zeta)\right\|_{2}^{2} \leq\left\|E_{A \rtimes \Gamma_{i}}(\zeta)\right\|_{2}^{2}=\sum_{\gamma \in \Gamma_{i}}\left\|E_{A}\left(\zeta u_{\gamma}^{*}\right)\right\|_{2}^{2}$. Hence, we can further reduce the claim to showing that $\lim _{n \rightarrow \omega}\left\|E_{A}\left(\eta_{1}^{*} y_{n} \xi_{1}\right)\right\|_{2}=0$, for all $\xi_{1}, \eta_{1} \in M$. This in turn is an immediate consequence of the fact that $y=\left(y_{n}\right)_{n} \perp A^{\omega} \rtimes \Gamma$.

Next, if $u \in \mathcal{U}(D)$, then by Claim 2 we get that $\delta_{t}(u) y \perp y \delta_{t}(u)$. Using Claim 1 we therefore derive that

$$
64 \varepsilon^{2} \geq\left\|\left[\delta_{t}(u), y\right]\right\|_{2}^{2}=\left\|\delta_{t}(u) y\right\|_{2}^{2}+\left\|y \delta_{t}(u)\right\|_{2}^{2} .
$$

In particular, we obtain that

$$
8 \varepsilon \geq\left\|\delta_{t}(u) y\right\|_{2}, \forall u \in \mathcal{U}(D) .
$$

Now, we use the same trick as in the proof of 39], Lemma 2.1. Since $E_{M}\left(\theta_{t}(u)\right) \in$ $M, u \in M, y \in M^{\omega}$ we get that $\beta\left(E_{M}\left(\theta_{t}(u)\right)\right)=E_{M}\left(\theta_{t}(u)\right), \beta(u)=u$ and $\beta(y)=y$. Thus

$$
\begin{aligned}
\left\|\theta_{-t}(u) y-E_{M}\left(\theta_{t}(u)\right) y\right\|_{2} & =\left\|\beta\left(\theta_{-t}(u) y-E_{M}\left(\theta_{t}(u)\right) y\right)\right\|_{2} \\
& =\left\|\beta\left(\theta_{-t}(u)\right) y-E_{M}\left(\theta_{t}(u)\right) y\right\|_{2} \\
& =\left\|\theta_{t}(\beta(u)) y-E_{M}\left(\theta_{t}(u)\right) y\right\|_{2} \\
& =\left\|\delta_{t}(u) y\right\|_{2} \leq 8 \varepsilon, \forall u \in \mathcal{U}(D) .
\end{aligned}
$$

By combining (3.c) and (3.d) we deduce that

$$
\left\|\theta_{t}(u) y-\theta_{-t}(u) y\right\|_{2} \leq 16 \varepsilon, \forall u \in \mathcal{U}(D) .
$$


Thus we have that

$$
\begin{aligned}
\Re \tau\left(\theta_{t}(u) y y^{*} \theta_{-t}\left(u^{*}\right)\right) & =\frac{1}{2}\left(\left\|\theta_{t}(u) y\right\|_{2}^{2}+\left\|\theta_{-t}(u) y\right\|_{2}^{2}-\left\|\theta_{t}(u) y-\theta_{-t}(u) y\right\|_{2}^{2}\right) \\
& \geq\|y\|_{2}^{2}-128 \varepsilon^{2}>0
\end{aligned}
$$

If $z=E_{\tilde{M}}\left(y y^{*}\right) \in \tilde{M}$, then $\tau\left(\theta_{t}(u) y y^{*} \theta_{-t}\left(u^{*}\right)\right)=\tau\left(\theta_{t}(u) z \theta_{-t}\left(u^{*}\right)\right)$; hence (3.f) implies that $\Re \tau\left(\theta_{t}(u) z \theta_{-t}\left(u^{*}\right)\right) \geq\|y\|_{2}^{2}-128 \varepsilon>0$, for all $u \in \mathcal{U}(D)$. A standard averaging argument provides $0 \neq w \in \tilde{M}$ such that $\theta_{t}(u) w=w \theta_{-t}(u)$, for all $u \in \mathcal{U}(D)$. Thus, if $v=\theta_{t}(w) \neq 0$, then $\theta_{2 t}(u) v=v u$, for all $u \in \mathcal{U}(D)$, which gives a contradiction.

Proof of Theorem 3.2. Assuming that (2) is false we will demonstrate that (1) holds true.

Set $\mathcal{M}=\tilde{M}_{1} \bar{\otimes} M_{2}$ and observe that $M \subset \mathcal{M} \subset \tilde{M}$. Let $\theta_{t}^{\prime}:=\theta_{t}^{1} \otimes \operatorname{id}_{M_{2}} \in$ $\operatorname{Aut}(\mathcal{M})$. Let $\beta \in \operatorname{Aut}\left(\tilde{M}_{1}\right)$ be defined as in Section 1.5. By Lemma 1.5.1 $\beta^{\prime}=$ $\beta \otimes \operatorname{id}_{M_{2}} \in \operatorname{Aut}(\mathcal{M})$ satisfies

$$
\beta_{\mid M}^{\prime}=1_{M}, \beta^{\prime 2}=1_{\mathcal{M}}, \beta^{\prime} \theta_{t}^{\prime} \beta^{\prime}=\theta_{-t}^{\prime}, \forall t \in \mathbb{R} .
$$

Denote $A_{1}=B_{1}^{\Gamma_{1}}$ and $A_{2}=B_{2}^{\Gamma_{2}}$. Observe that we can write $M=\left(A_{1} \bar{\otimes} M_{2}\right) \rtimes \Gamma_{1}$, where $\Gamma_{1}$ acts by Bernoulli shifts on $A_{1}$ and trivially on $M_{2}$. Similarly, we have $M=\left(M_{1} \bar{\otimes} A_{2}\right) \rtimes \Gamma_{2}$.

Claim 1. There exists a family $\left\{C_{i}\right\}_{i \in I}$ of von Neumann subalgebras of $M$ and a family $\left\{\Gamma_{i}\right\}_{i \in I}$ of finite subgroups of $\Gamma_{1}$ such that $C_{i} \subset\left(A_{1} \bar{\otimes} M_{2}\right) \rtimes \Gamma_{i}$, for all $i \in I$, and

$$
L^{2}(\mathcal{M}) \ominus L^{2}(M) \cong \bigoplus_{i \in I} L^{2}\left(\left\langle M, e_{C_{i}}\right\rangle\right)
$$

as Hilbert $M$-bimodules.

Proof of Claim 1. The proof of [8], Lemma 5 provides a family $\left\{A_{i}\right\}_{i \in I}$ of von Neumann subalgebras of $M_{1}$ and a family $\left\{\Gamma_{i}\right\}_{i \in I}$ of finite subgroups of $\Gamma_{1}$ such that $A_{i} \subset A \rtimes \Gamma_{i}$, for all $i \in I$, and $L^{2}\left(\tilde{M}_{1}\right) \ominus L^{2}\left(M_{1}\right) \cong \bigoplus_{i \in I} L^{2}\left(\left\langle M_{1}, e_{A_{i}}\right\rangle\right)$, as Hilbert $M_{1}$-bimodules. By letting $C_{i}=A_{i} \bar{\otimes} M_{2} \subset\left(A_{1} \rtimes \Gamma_{i}\right) \bar{\otimes} M_{2}=\left(A_{1} \bar{\otimes} M_{2}\right) \rtimes \Gamma_{i}$ and using the decomposition $L^{2}(\mathcal{M}) \ominus L^{2}(M)=\left(L^{2}\left(\tilde{M}_{1}\right) \ominus L^{2}\left(M_{1}\right)\right) \bar{\otimes} L^{2}\left(M_{2}\right)$, the claim follows.

Now, recall that $\theta_{t}=\theta_{t}^{1} \otimes \theta_{t}^{2} \in \operatorname{Aut}\left(\tilde{M}=\tilde{M}_{1} \bar{\otimes} \tilde{M}_{2}\right)$ and that $\tilde{M} \supset \mathcal{M} \supset M$.

Claim 2. For every $x \in M$ and all $t \in \mathbb{R}$, we have that $\left\|\theta_{t}^{\prime}(x)-x\right\|_{2} \leq 2\left\|\theta_{\frac{t}{2}}(x)-x\right\|_{2}$.

Proof of Claim 2. Fix $x \in M$. We show first that $\left\|E_{M}\left(\theta_{t}^{\prime}(x)\right)\right\|_{2} \geq\left\|E_{M}\left(\theta_{t}(x)\right)\right\|_{2}$. Fix $t \in \mathbb{R}$ and for $i \in\{1,2\}$, denote by $T_{i}$ the bounded operator on $L^{2}\left(M_{i}\right)$ induced by $E_{M_{i}} \circ \theta_{t}^{i}: M_{i} \rightarrow M_{i}$.

Then $T_{i}$ is a contraction; hence $T_{i}^{*} T_{i} \leq 1$, as operators on $L^{2}\left(M_{i}\right)$. Also, we have that $E_{M}\left(\theta_{t}^{\prime}(x)\right)=\left(T_{1} \otimes 1\right)(x)$ and $E_{M}\left(\theta_{t}(x)\right)=\left(T_{1} \otimes T_{2}\right)(x)$. Our assertion now follows from the following estimate: $\left\|\left(T_{1} \otimes T_{2}\right)(x)\right\|_{2}^{2}=\left\langle\left(T_{1}^{*} T_{1} \otimes T_{2}^{*} T_{2}\right)(x), x\right\rangle \leq$ $\left\langle\left(T_{1}^{*} T_{1} \otimes 1\right)(x), x\right\rangle=\left\|\left(T_{1} \otimes 1\right)(x)\right\|_{2}^{2}$.

Next, since $x \in M$, by using (3.g) and [39], Lemma 2.1 we deduce that $\| \theta_{t}^{\prime}(x)-$ $x\left\|_{2} \leq 2\right\| \theta_{\frac{t}{2}}^{\prime}(x)-E_{M}\left(\theta_{\frac{t}{2}}^{\prime}(x)\right) \|_{2}$. In combination with the above assertion we get 
that

$$
\begin{aligned}
& \left\|\theta_{t}^{\prime}(x)-x\right\|_{2}^{2} \leq 4\left\|\theta_{\frac{t}{2}}^{\prime}(x)-E_{M}\left(\theta_{\frac{t}{2}}^{\prime}(x)\right)\right\|_{2}^{2}=4\left(\left\|\theta_{\frac{t}{2}}^{\prime}(x)\right\|_{2}^{2}-\left\|E_{M}\left(\theta_{\frac{t}{2}}^{\prime}(x)\right)\right\|_{2}^{2}\right) \\
& \quad \leq 4\left(\|x\|_{2}^{2}-\left\|E_{M}\left(\theta_{\frac{t}{2}}(x)\right)\right\|_{2}^{2}=4\left\|x-E_{M}\left(\theta_{\frac{t}{2}}(x)\right)\right\|_{2}^{2} \leq 4\left\|\theta_{\frac{t}{2}}(x)-x\right\|_{2}^{2}\right.
\end{aligned}
$$

and the claim is proven.

Since $x=\left(x_{n}\right)_{n} \in D^{\prime} \cap M^{\omega}$ satisfies $\lim _{t \rightarrow 0}\left\|\theta_{t}(x)-x\right\|_{2}=0$, by Claim 2 we conclude that $\lim _{t \rightarrow 0}\left\|\theta_{t}^{\prime}(x)-x\right\|_{2}=0$. Claim 1 says that we are in a position to apply Lemma 3.3 and deduce that one of the following happens: $\left(x_{n}\right)_{n} \in\left(A_{1} \bar{\otimes} M_{2}\right)^{\omega} \rtimes \Gamma_{1}$ or there exists $t \neq 0$ and $0 \neq v \in \mathcal{M}$ such that $\theta_{t}^{\prime}(y) v=v y$, for all $y \in D$. If the latter is true, since $\theta_{t}^{\prime}=\theta_{t}^{1} \otimes \mathrm{id}_{M_{2}}$, Theorem 2.1 and Remark 2.2 imply that $D \prec_{M} L\left(\Gamma_{1}\right) \bar{\otimes} M_{2}$. This contradicts our assumption that (2) is false, so we must have that

$$
\left(x_{n}\right)_{n} \in\left(A_{1} \bar{\otimes} M_{2}\right)^{\omega} \rtimes \Gamma_{1} .
$$

Now, let $\mathcal{M}=M_{1} \bar{\otimes} \tilde{M}_{2}$ and $\theta_{t}^{\prime}=\operatorname{id}_{M_{1}} \otimes \theta_{t}^{2} \in \operatorname{Aut}(\mathcal{M})$. By arguing in the same way as above and using the fact that (2) is false, we get that

$$
\left(x_{n}\right)_{n} \in\left(M_{1} \bar{\otimes} A_{2}\right)^{\omega} \rtimes \Gamma_{2} .
$$

Finally, we show that (3.h) and (3.i) together imply that $\left(x_{n}\right)_{n} \in A^{\omega} \rtimes \Gamma$.

For finite sets $F_{1} \subset \Gamma_{1}$ and $F_{2} \subset \Gamma_{2}$, denote by $Q_{F_{1}}$ and $R_{F_{2}}$ the orthogonal projections from $L^{2}(M)$ onto the closed linear span of $\left\{A_{1} u_{\gamma} \otimes M_{2} \mid \gamma \in F_{1}\right\}$ and of $\left\{M_{1} \otimes A_{2} u_{\gamma} \mid \gamma \in F_{2}\right\}$, respectively.

Then (3.h) and (3.i) can be rewritten as

$$
\inf _{F_{1} \subset \Gamma_{1} \text { finite }} \lim _{n \rightarrow \omega}\left\|x_{n}-Q_{F_{1}}\left(x_{n}\right)\right\|_{2}=0, \inf _{F_{2} \subset \Gamma_{2} \text { finite }} \lim _{n \rightarrow \omega}\left\|x_{n}-R_{F_{2}}\left(x_{n}\right)\right\|_{2}=0 .
$$

Thus, we have that $\inf _{F_{1} \subset \Gamma_{1}, F_{2} \subset \Gamma_{2} \text { finite }} \lim _{n \rightarrow \omega}\left\|x_{n}-\left(R_{F_{2}} \circ Q_{F_{1}}\right)\left(x_{n}\right)\right\|_{2}=0$. Since $R_{F_{2}} \circ Q_{F_{1}}$ is the orthogonal projection from $L^{2}(M)$ onto the closed linear span of $\left\{\left(A_{1} \otimes A_{2}\right) u_{\gamma} \mid \gamma \in F_{1} \times F_{2}\right\}$, it follows that $\left(x_{n}\right)_{n} \in A^{\omega} \rtimes \Gamma$; therefore (1) holds true.

Remark 3.4. In the sequel, we will only use the following corollary of Theorem 3.2: let $D \subset M$ be an abelian von Neumann subalgebra such that condition (2) is false and suppose that $u_{n} \in L(\Gamma)$ is a sequence of unitaries which normalize $D$ and satisfy $\left\|E_{L\left(\Gamma_{1}\right)}\left(a u_{n} b\right)\right\|_{2},\left\|E_{L\left(\Gamma_{2}\right)}\left(a u_{n} b\right)\right\|_{2} \rightarrow 0$, for every $a, b \in L(\Gamma)$. Then for all $x \in D$ we have that $\left(u_{n} x u_{n}^{*}\right)_{n} \in A^{\omega} \rtimes_{\sigma} \Gamma$. Since $\theta_{t}\left(u_{n}\right)=u_{n}$, for all $t$ and $n$, this statement is indeed a consequence of Theorem 3.2.

This corollary can be alternatively proved by adapting Popa's "clustering coefficients" method (see [34]).

Following the strategy from [34], one first shows that the Fourier coefficients of $x_{n}=u_{n} x u_{n}^{*}$ cluster (i.e., they asymptotically belong to $B_{1}^{\Gamma_{1} \backslash F_{1}} \bar{\otimes} B_{2}^{\Gamma_{2} \backslash F_{2}}$ for any finite sets $F_{1} \subset \Gamma_{1}$ and $\left.F_{2} \subset \Gamma_{2}\right)$. Second, one uses the fact that the clustering sequence $\left(x_{n}\right)_{n} \in M^{\omega}$ commutes with elements $y \in D$ which are "almost perpendicular" onto both $M_{1} \bar{\otimes} L\left(\Gamma_{2}\right)$ and $L\left(\Gamma_{2}\right) \bar{\otimes} M_{2}$ to conclude that $\left(x_{n}\right)_{n} \in A^{\omega} \rtimes_{\sigma} \Gamma$.

We end this section by showing that condition (2) in Theorem 3.2 can be exploited to get information on the quasi-normalizer of $D$.

The next lemma is a straightforward generalization of [33], Theorem 3.1, but we include a proof for the reader's convenience. 
Proposition 3.5 ([33]). Let $\Gamma \curvearrowright(X, \mu)$ be a p.m.p. mixing action and denote $M=L^{\infty}(X) \rtimes \Gamma$. Let $N$ be a finite von Neumann algebra. Assume that $D$ is a von Neumann subalgebra of $M \bar{\otimes} N$ such that $D \prec_{M \bar{\otimes} N} L(\Gamma) \bar{\otimes} N$. Denote by $P$ the von Neumann algebra generated by the quasi-normalizer of $D$ in $M \bar{\otimes} N$. If $D \nprec_{M \bar{\otimes} N} 1 \otimes N$, then $P \prec_{M \bar{\otimes} N} L(\Gamma) \bar{\otimes} N$.

Proof. We first claim that if $p \in L(\Gamma) \bar{\otimes} N$ is a projection and $D \subset p(L(\Gamma) \bar{\otimes} N) p$ is a unital von Neumann subalgebra such that $D \nprec_{L(\Gamma) \bar{\otimes} N} 1 \otimes N$, then $q \mathcal{N}_{p(M \bar{\otimes} N) p}(D) \subset$ $p(L(\Gamma) \bar{\otimes} N) p$. By [33, Section 3 (or [17, proof of Theorem 1.1) in order to prove the claim it suffices to show that for every $\varepsilon>0, \eta_{1}, \ldots, \eta_{k} \in(M \bar{\otimes} N) \ominus(L(\Gamma) \bar{\otimes} N)$, we can find $u \in \mathcal{U}(D)$ such that $\left\|E_{L(\Gamma) \bar{\otimes} N}\left(\eta_{i} u \eta_{j}^{*}\right)\right\|_{2} \leq \varepsilon$, for all $i, j \in\{1, \ldots, k\}$.

Since $E_{L(\Gamma) \bar{\otimes} N}$ is $L(\Gamma) \bar{\otimes} N$-bimodular, by using Kaplansky's density theorem it is enough to prove the last assertion for $\eta_{i} \in L^{\infty}(X) \otimes 1$ with $\tau\left(\eta_{i}\right)=0$ and $\left\|\eta_{i}\right\| \leq 1$.

Next, decompose $u \in \mathcal{U}(D)$ as $u=\sum_{\gamma} u_{\gamma} \otimes x_{\gamma}$, where $x_{\gamma} \in N$. Denote by $\alpha$ the induced action of $\Gamma$ on $L^{\infty}(X)$. We have that

$$
\left\|E_{L(\Gamma) \bar{\otimes} N}\left(\eta_{i} u \eta_{j}^{*}\right)\right\|_{2}^{2}=\sum_{\gamma \in \Gamma}\left|\tau\left(\eta_{i} \alpha_{\gamma}\left(\eta_{j}^{*}\right)\right)\right|^{2}\left\|x_{\gamma}\right\|_{2}^{2}
$$

Since $\alpha$ is mixing and $\tau\left(\eta_{i}\right)=0$, for all $i$, we can find $F \subset \Gamma$ finite such that

$$
\left|\tau\left(\eta_{i} \alpha_{\gamma}\left(\eta_{j}^{*}\right)\right)\right| \leq \frac{\varepsilon}{2}, \forall i, j \in\{1, \ldots, k\}, \forall \gamma \in \Gamma \backslash F .
$$

Since $D \nprec_{L(\Gamma) \bar{\otimes} N} 1 \otimes N$, by Popa's theorem we can find $u \in \mathcal{U}(D)$ such that

$$
\sum_{\gamma \in F}\left\|x_{\gamma}\right\|_{2}^{2}=\sum_{\gamma \in F}\left\|E_{1 \otimes N}\left(u\left(u_{\gamma}^{*} \otimes 1\right)\right)\right\|_{2}^{2} \leq \frac{\varepsilon}{2} .
$$

As $\left|\tau\left(\eta_{i} \alpha_{\gamma}\left(\eta_{j}^{*}\right)\right)\right| \leq\left\|\eta_{i}\right\|\left\|\eta_{j}\right\| \leq 1$, for all $\gamma \in \Gamma$, it is clear that the combination of (3.j), (3.k) and (3.l) implies that $\left\|E_{L(\Gamma) \bar{\otimes} N}\left(\eta_{i} u \eta_{j}^{*}\right)\right\|_{2} \leq \varepsilon$, for all $i, j \in\{1, \ldots, k\}$, as claimed.

Going back to the general case, let $D$ be a von Neumann algebra such that $D \prec_{M \bar{\otimes} N} L(\Gamma) \bar{\otimes} N$ but $D \nprec_{M \bar{\otimes} N} 1 \otimes N$. By [45, Remark 3.8 we can find non-zero projections $q \in D, p \in L(\Gamma) \bar{\otimes} N$, a $*$-homomorphism $\psi: q D q \rightarrow p(L(\Gamma) \bar{\otimes} N) p$ and a non-zero partial isometry $v \in p(M \bar{\otimes} N) q$ such that $\psi(x) v=v x$, for all $x \in q D q$, and $\psi(q D q) \nprec_{L(\Gamma) \bar{\otimes} N} 1 \otimes N$. By the first part of the proof (applied to $\psi(q D q)$ ) we deduce that $\psi(q D q)^{\prime} \cap p(L(\Gamma) \bar{\otimes} N) p \subset p(L(\Gamma) \bar{\otimes} N) p$; hence $v v^{*} \in p(L(\Gamma) \bar{\otimes} N) p$. This further implies that $v D v^{*} \subset L(\Gamma) \bar{\otimes} N$. Since $v^{*} v \in(q D q)^{\prime} \cap q(M \bar{\otimes} N) q$, we can find $q^{\prime} \in D^{\prime} \cap(M \bar{\otimes} N) \subset P$ such that $v^{*} v=q q^{\prime}$. Let $u$ be any unitary element extending $v$. Then $u\left(q D q q^{\prime}\right) u^{*}=v D v^{*} \subset L(\Gamma) \bar{\otimes} N$.

Finally, since $D$ is quasi-regular in $P$, by [33, Lemma 3.5 we get that $q D q q^{\prime}$ is quasi-regular in $q q^{\prime} P q q^{\prime}$. Also, from the hypothesis we have that no corner of $q D q q^{\prime}$ embeds into $1 \otimes N$. Thus, by the first part of the proof, we deduce that $u\left(q q^{\prime} P q q^{\prime}\right) u^{*} \subset L(\Gamma) \bar{\otimes} N$; hence a corner of $P$ embeds into $L(\Gamma) \bar{\otimes} N$.

\section{LOWER BOUND ON HEIGHT}

Let $M=A \rtimes \Gamma$ be a $\mathrm{II}_{1}$ factor associated with a Bernoulli action. For every $v \in L(\Gamma)$, we define the height of $v$ as $h(v)=\max _{\gamma \in \Gamma}\left|\tau\left(v u_{\gamma}^{*}\right)\right|$. Consider a sequence of elements $\left\{v_{n}\right\}_{n \geq 1} \subset(L(\Gamma))_{1}$. In this section, we provide a set of conditions which guarantee that the heights of the $v_{n}$ 's are uniformly bounded away from 0 . 
Theorem 4.1. Assume the notation from 3.1, i.e. $M_{i}=B_{i}^{\Gamma_{i}} \rtimes \Gamma_{i}, M=M_{1} \bar{\otimes} M_{2}$, $A=B_{1}^{\Gamma_{1}} \bar{\otimes} B_{2}^{\Gamma_{2}}, \Gamma=\Gamma_{1} \times \Gamma_{2}$. Let $\left\{v_{n}\right\}_{n \geq 1} \subset(L(\Gamma))_{1}$ be a sequence for which there exist $C, c>0$ and $x \in M$ such that $C>\sqrt{2} c$,

(1) $\left\|E_{A^{\omega} \rtimes \Gamma}\left(\left(v_{n} x v_{n}^{*}\right)_{n}\right)\right\|_{2} \geq C$,

(2) $\left\|E_{M_{1} \bar{\otimes} L\left(\Gamma_{2}\right)}(x)\right\|_{2} \leq c$ and

(3) $\left\|E_{L\left(\Gamma_{1}\right) \bar{\otimes} M_{2}}(x)\right\|_{2} \leq c$.

Then $\lim _{n \rightarrow \omega} h\left(v_{n}\right)>0$.

Proof. Let $\varepsilon>0$ such that $C-\sqrt{2} c-3 \varepsilon>0$ and let $x \in M$ satisfy conditions (1)-(3). The first condition yields a finite set $F \subset \Gamma$ such that if $P_{F}$ denotes the orthogonal projection from $L^{2}(M)$ onto the closed linear span of $\left\{A u_{\gamma} \mid \gamma \in F\right\}$, then

$$
\lim _{n \rightarrow \omega}\left\|P_{F}\left(v_{n} x v_{n}^{*}\right)\right\|_{2} \geq C-\varepsilon .
$$

Next, let $E_{1}=E_{M_{1} \bar{\otimes} L\left(\Gamma_{2}\right)}$ and $E_{2}=E_{L\left(\Gamma_{1}\right) \bar{\otimes} M_{2}}$. Since $E_{1}$ and $E_{2}$ commute, $y=\left(\left(\operatorname{id}_{M}-E_{1}\right) \circ\left(\operatorname{id}_{M}-E_{2}\right)\right)(x)$ satisfies $y \perp M_{1} \bar{\otimes} L\left(\Gamma_{2}\right)$ and $y \perp L\left(\Gamma_{1}\right) \bar{\otimes} M_{2}$. Thus, if we write $y=\sum_{\gamma \in \Gamma} a_{\gamma} u_{\gamma}$, where $a_{\gamma} \in A$, then $E_{B_{1}^{\Gamma_{1} \otimes 1}}\left(a_{\gamma}\right)=E_{1 \otimes B_{2}^{\Gamma_{2}}}\left(a_{\gamma}\right)=0$, for all $\gamma \in \Gamma$.

Also, conditions (2) and (3) yield $\|x-y\|_{2}=\left\|E_{1}(x)+\left(E_{2}(x)-\left(E_{1} \circ E_{2}\right)(x)\right)\right\|_{2} \leq$ $\sqrt{2} c$.

Let $K \subset \Gamma$ be finite such that $z=P_{K}(y)$ satisfies $\|z-y\|_{2} \leq \varepsilon$. Let $S_{1} \subset$ $\Gamma_{1}, S_{2} \subset \Gamma_{2}$ be finite such that if $b_{\gamma}=E_{B_{1}^{S_{1}} \bar{\otimes} B_{2}^{S_{2}}}\left(a_{\gamma}\right)$, then $w=\sum_{\gamma \in K} b_{\gamma} u_{\gamma}$ satisfies $\|w-z\|_{2} \leq \varepsilon$.

The triangle inequality implies that $\|w-x\|_{2} \leq \sqrt{2} c+\|p\|_{2}+2 \varepsilon$ and hence by (4.a) we have

$$
\lim _{n \rightarrow \omega}\left\|P_{F}\left(v_{n} w v_{n}^{*}\right)\right\|_{2} \geq(C-\varepsilon)-(\sqrt{2} c+2 \varepsilon)>0 .
$$

Let $S=S_{1} S_{1}^{-1} \times S_{2} S_{2}^{-1} \subset \Gamma$, where $S_{1} S_{1}^{-1}=\left\{g h^{-1} \mid g, h \in S_{1}\right\}$. We claim that

$$
\tau\left(\sigma_{\gamma}\left(b_{g}\right) b_{h}^{*}\right)=0, \forall \gamma \in \Gamma \backslash S, \forall g, h \in K .
$$

Indeed, let $\gamma=\left(\gamma_{1}, \gamma_{2}\right) \in \Gamma \backslash S$ and assume that $\gamma_{1} \notin S_{1} S_{1}^{-1}$ (the case $\gamma_{2} \notin S_{2} S_{2}^{-1}$ being analogous). Let $g, h \in K$. Since $b_{h} \in B_{1}^{S_{1}} \bar{\otimes} B_{2}^{\Gamma_{2}}$ and $\gamma_{1} S_{1} \cap S_{1}=\emptyset$, we have $E_{B_{1}^{\gamma_{1} S_{1}} \bar{\otimes} B_{2}^{\Gamma_{2}}}\left(b_{h}\right)=E_{1 \otimes B_{2}^{\Gamma_{2}}}\left(b_{h}\right)=E_{1 \otimes B_{2}^{S_{2}}}\left(a_{h}\right)=0$. Since $\sigma_{\gamma}\left(b_{g}\right) \in B_{1}^{\gamma_{1} S_{1}} \bar{\otimes} B_{2}^{\Gamma_{2}}$, we derive that $\tau\left(\sigma_{\gamma}\left(b_{g}\right) b_{h}^{*}\right)=\tau\left(\sigma_{\gamma}\left(b_{g}\right) E_{B_{1}^{\gamma_{1} S_{1}} \bar{\otimes} B_{2}^{\Gamma_{2}}}\left(b_{h}\right)^{*}\right)=0$, which proves (4.c).

Next, fix $v \in L(\Gamma)$ and estimate $\left\|P_{F}\left(v w v^{*}\right)\right\|_{2}$. Let $\delta=\max _{g, h \in K}\left\|b_{g}\right\|_{2}\left\|b_{h}\right\|_{2}$ and write $v=\sum_{\gamma \in \Gamma} c_{\gamma} u_{\gamma}$, where $c_{\gamma} \in \mathbb{C}$, for all $\gamma \in \Gamma$. Then

$$
v w v^{*}=\sum_{\gamma, \gamma^{\prime} \in \Gamma, g \in K} c_{\gamma} \overline{c_{\gamma^{\prime}}} \sigma_{\gamma}\left(b_{g}\right) u_{\gamma g \gamma^{\prime-1}}=\sum_{h \in \Gamma}\left(\sum_{\gamma \in \Gamma, g \in K} c_{\gamma} \overline{c_{h^{-1} \gamma g}} \sigma_{\gamma}\left(b_{g}\right)\right) u_{h} .
$$

Thus,

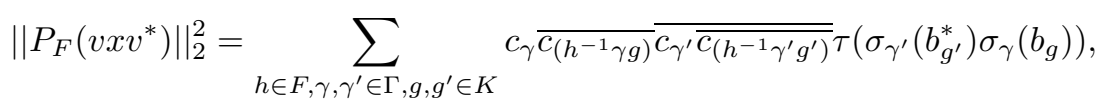

which by (4.c) is further equal to the real part of

$$
\sum_{\gamma \in \Gamma, s \in S, h \in F, g, g^{\prime} \in K} c_{\gamma} \overline{c_{\left(h^{-1} \gamma g\right)} c_{\gamma s^{-1}}} c_{\left(h^{-1} \gamma s^{-1} g^{\prime}\right)} \tau\left(\left(b_{g^{\prime}}^{*}\right) \sigma_{s}\left(b_{g}\right)\right) .
$$


Since $\mathcal{R} e\left(a_{1} a_{2} a_{3} a_{4}\right) \leq \frac{1}{4} \sum_{i=1}^{4}\left|a_{i}\right|^{4}$, for every $\left\{a_{i}\right\}_{i=1}^{4} \in \mathbb{C}$, the last term is majorized by

$$
\begin{aligned}
\frac{\delta}{4}{ }_{\gamma \in \Gamma, s \in S, h \in F, g, g^{\prime} \in K}\left(\left|c_{\gamma}\right|^{4}+\left|c_{\left(h^{-1} \gamma g\right)}\right|^{4}+\left|c_{\gamma s^{-1}}\right|^{4}+\left|c_{\left(h^{-1} \gamma s^{-1} g^{\prime}\right)}\right|^{4}\right) \\
\leq \frac{\delta}{4}(1+|S|)(1+|F||K|) \sum_{\gamma \in \Gamma}\left|c_{\gamma}\right|^{4} \\
\leq \frac{\delta}{4}(1+|S|)(1+|F||K|) \max _{\gamma \in \Gamma}\left|c_{\gamma}\right|^{2} \sum_{\gamma^{\prime} \in \Gamma}\left|c_{\gamma^{\prime}}\right|^{2} \\
=\frac{\delta}{4}(1+|S|)(1+|F||K|) h(v)^{2}|| v \|_{2}^{2} .
\end{aligned}
$$

Altogether, we have shown that there exists a constant $\kappa>0$ such that $h(v)\|v\|_{2}$ $\geq \kappa\left\|P_{F}\left(v w v^{*}\right)\right\|_{2}$, for all $v \in L(\Gamma)$. Since $\left\|v_{n}\right\| \leq 1$ we get that $h\left(v_{n}\right) \geq$ $\kappa\left\|P_{F}\left(v_{n} w v_{n}^{*}\right)\right\|_{2}$, for all $n$, and (4.b) implies the conclusion of the theorem.

Remark 4.2. If $M=A \rtimes_{\sigma} \Gamma$ is a $\mathrm{II}_{1}$ factor coming from a Bernoulli action, then the above proof shows the following: assume that $\left\{v_{n}\right\}_{n>1} \subset(L(\Gamma))_{1}$ is a sequence for which there exists $x \in M$ such that $\left\|E_{A^{\omega} \rtimes \Gamma}\left(\left(v_{n} x v_{n}^{*}\right)_{n}\right)\right\|_{2}>\left\|E_{L(\Gamma)}(x)\right\|_{2}$. Then $\lim _{n \rightarrow \omega} h\left(v_{n}\right)>0$.

\section{A CONJUGACY RESUlt FOR SUBALGEBRAS}

Let $M=B^{\Gamma} \rtimes \Gamma$ be a $\mathrm{II}_{1}$ factor coming from a Bernoulli action. In this section, we give a criterion for proving that a von Neumann subalgebra $C$ of $M$ has a corner which embeds into $B^{\Gamma}$. More generally, our criterion applies to subalgebras of $M \bar{\otimes} N$, where $N$ is an arbitrary finite von Neumann algebra.

Before stating it we need to introduce some notation.

Notation. We consider the orthogonal projections onto certain Hilbert subspaces of $L^{2}(M \bar{\otimes} N)$. For subsets $S, F$ and $G$ of $\Gamma$ we denote by:

- $P_{S}$ the orthogonal projection onto the closed linear span of $\left\{B^{\Gamma} u_{\gamma} \otimes N \mid \gamma \in S\right\}$.

- $\mathcal{H}_{F}$ the closed linear span of $\left\{B^{F} u_{\gamma} \otimes N \mid \gamma \in \Gamma\right\}$.

- $Q_{F}$ the orthogonal projection onto $\mathcal{H}_{F}$.

- $Q_{F}^{0}:=Q_{F}-Q_{\emptyset}$.

- $Q_{F}^{G}:=Q_{F \cup G}-Q_{G}$ the orthogonal projection onto $\mathcal{H}_{F}^{G}:=\mathcal{H}_{F \cup G} \ominus \mathcal{H}_{G}$.

Next, we record some useful boundedness and modularity properties of these projections.

Lemma 5.1. Let $S, F, G \subset \Gamma$ be finite sets. Then $P_{S}$ commutes with $Q_{F}, Q_{F}^{G}$ and

$$
\left\|P_{S}(x)\right\| \leq|S|,\left\|\left(P_{S} \circ Q_{F}\right)(x)\right\| \leq|S|,\left\|\left(P_{S} \circ Q_{F}^{G}\right)(x)\right\| \leq 2|S|, \forall x \in(M \bar{\otimes} N)_{1} .
$$

If $F$ contains $S G$, then $Q_{F}\left(P_{S}(x) y\right)=Q_{F}\left(P_{S}(x)\right) y$ for every $x \in M \bar{\otimes} N$ and $y \in \mathcal{H}_{G}$.

Proof. The commutativity assertion is trivial. Let $x \in M \bar{\otimes} N$ with $\|x\| \leq 1$. Write $M \bar{\otimes} N=\left(B^{\Gamma} \bar{\otimes} N\right) \rtimes_{\rho} \Gamma$, where $\Gamma$ acts through Bernoulli action on $B^{\Gamma}$ and trivially on $N$. Decompose $x=\sum_{\gamma \in \Gamma} x_{\gamma} u_{\gamma}$, where $x_{\gamma}=E_{B^{\Gamma} \bar{\otimes} N}\left(x u_{\gamma}^{*}\right)$. Then $\left\|x_{\gamma}\right\| \leq 1$, for all $\gamma \in \Gamma$. Since $P_{S}(x)=\sum_{\gamma \in S} x_{\gamma} u_{\gamma},\left(P_{S} \circ Q_{F}\right)(x)=\sum_{\gamma \in S} E_{B^{F} \bar{\otimes} N}\left(x_{\gamma}\right) u_{\gamma}$ and $Q_{F}^{G}=Q_{F \cup G}-Q_{G}$, the three inequalities follow. 
For the last assertion, it suffices to show that if $F \supset S G$ and if $x=\sum_{\gamma \in S} x_{\gamma} u_{\gamma}$ and $y=\sum_{g \in \Gamma} y_{g} u_{g}$ with $x_{\gamma} \in B^{\Gamma \bar{\otimes}} N$ and $y_{g} \in B^{G \bar{\otimes}} N$, for all $\gamma \in S$ and $g \in \Gamma$, then $Q_{F}(x y)=Q_{F}(x) y$. Notice that if $\gamma \in S$, then $\rho_{\gamma}\left(y_{g}\right) \in B^{\gamma G \bar{\otimes}} \subset \subset B^{F \bar{\otimes}} N$, for all $g \in \Gamma$. Thus, we have that

$$
\begin{aligned}
Q_{F}(x y) & =\sum_{\gamma \in S, g \in \Gamma} E_{B^{F} \bar{\otimes} N}\left(x_{\gamma} \rho_{\gamma}\left(y_{g}\right)\right) u_{\gamma g} \\
& =\sum_{\gamma \in S, g \in \Gamma} E_{B^{F} \bar{\otimes} N}\left(x_{\gamma}\right) \rho_{\gamma}\left(y_{g}\right) u_{\gamma g}=Q_{F}(x) y .
\end{aligned}
$$

We are now ready to state and prove the main result of this section.

Theorem 5.2. Let $\Gamma$ be a countable group, $B$ be a finite von Neumann algebra and denote $M=B^{\Gamma} \rtimes \Gamma$. Let $N$ be a finite von Neumann algebra and $p \in M \bar{\otimes} N$ be a projection. Let $C \subset p(M \bar{\otimes} N) p$ be a unital von Neumann subalgebra. Suppose that there exist a sequence $\left\{x_{n}\right\}_{n \geq 1} \subset \mathcal{U}(p(M \bar{\otimes} N) p)$ and finite subsets $S,\left\{F_{n}\right\}_{n \geq 1}$ of $\Gamma$ such that

- $\left\|x_{n} y-y x_{n}\right\|_{2} \rightarrow 0$, for all $y \in C$,

- $F_{n} \rightarrow \infty$, as $n \rightarrow \infty$ (i.e. if $\gamma \in \Gamma$, then $\gamma \notin F_{n}$, for large enough $n$ ),

- $\sup _{n \geq 1}\left|F_{n}\right|<\infty$, and

- $\lim \sup _{n \rightarrow \infty}\left(\left\|x_{n}-Q_{F_{n}}^{0}\left(x_{n}\right)\right\|_{2}+3\left\|x_{n}-P_{S}\left(x_{n}\right)\right\|_{2}\right)<\|p\|_{2}$.

Then $C \prec_{M \bar{\otimes} N} B^{\Gamma \bar{\otimes}} N$.

Proof. By replacing $\left(x_{n}\right)_{n}$ with a subsequence, we may assume $\left\|x_{n}-Q_{F_{n}}^{0}\left(x_{n}\right)\right\|_{2}$ $\leq c_{1}$ and $\left\|x_{n}-P_{S}\left(x_{n}\right)\right\|_{2} \leq c_{2}$, for all $n$, for some $c_{1}, c_{2} \geq 0$ satisfying $c_{1}+3 c_{2}<$ $\|p\|_{2}$. Let $\varepsilon>0$ such that $c=\|p\|_{2}-\left(c_{1}+3 c_{2}+11 \varepsilon\right)>0$.

We begin with the following:

Claim. Let $y \in \mathcal{U}(C)$ and $G \subset \Gamma$ be a finite subset such that $\left\|y-Q_{G}(y)\right\|_{2} \leq \frac{\varepsilon}{|S|}$. Let $K_{n} \subset \Gamma$ be a sequence of finite subsets such that $\left\|y-P_{K_{n}}(y)\right\|_{2} \leq \frac{\varepsilon}{|S|}$ and $K_{n} F_{n}$ is disjoint from $G \cup S G$. Then we can find $N \geq 1$ such that $\left\|Q_{K_{n} F_{n}}^{G \cup S G}\left(x_{n}\right)\right\|_{2} \geq c$, for all $n \geq N$.

Proof of Claim. Let $y_{n}=\left(P_{K_{n}} \circ Q_{G}\right)(y)$. Then $\left\|y_{n}-y\right\|_{2} \leq \frac{2 \varepsilon}{|S|}$. Also, let $x_{n}^{\prime}=$ $\left(P_{S} \circ Q_{F_{n}}^{0}\right)\left(x_{n}\right)$. Since $P_{S}$ and $Q_{F_{n}}^{0}$ commute, we get that

$$
\left\|x_{n}-x_{n}^{\prime}\right\|_{2}=\left\|x_{n}-Q_{F_{n}}^{0}\left(x_{n}\right)+Q_{F_{n}}^{0}\left(x_{n}-P_{S}\left(x_{n}\right)\right)\right\|_{2} \leq c_{1}+c_{2} .
$$

Now, $y_{n} x_{n}^{\prime}$ belongs to the closed linear span of $\left\{B^{G} u_{k}\left(B^{F_{n}} \ominus \mathbb{C} 1\right) u_{\gamma}\right) \otimes N \mid k \in$ $\left.K_{n}, \gamma \in S\right\}$. Since $u_{k}\left(B^{F_{n}} \ominus \mathbb{C} 1\right) \subset\left(B^{K_{n} F_{n}} \ominus \mathbb{C} 1\right) u_{k}$, for $k \in K_{n}$, we get that $y_{n} x_{n}^{\prime} \in \mathcal{H}_{K_{n} F_{n}}^{G}$ (recall that $\mathcal{H}_{F}^{G}=\mathcal{H}_{F \cup G} \ominus \mathcal{H}_{G}$ ). Since $K_{n} F_{n}$ is disjoint from $G \cup S G$, we get that $y_{n} x_{n}^{\prime} \in \mathcal{H}_{K_{n} F_{n}}^{G \cup S G}$.

Next, we estimate $\left\|y x_{n}-y_{n} x_{n}^{\prime}\right\|_{2}$. Lemma 5.1 gives that $\left\|x_{n}^{\prime}\right\| \leq 2|S|$; hence $\left\|y-y_{n}\right\|_{2}\left\|x_{n}^{\prime}\right\| \leq 4 \varepsilon$. By using the triangle inequality and (5.a) we get that

$$
\left\|y x_{n}-y_{n} x_{n}^{\prime}\right\|_{2} \leq\|y\|\left\|x_{n}-x_{n}^{\prime}\right\|_{2}+\left\|y-y_{n}\right\|_{2}\left\|x_{n}^{\prime}\right\| \leq c_{1}+c_{2}+4 \varepsilon .
$$

Since $y_{n} x_{n}^{\prime} \in \mathcal{H}_{K_{n} F_{n}}^{G \cup S G}$, (5.b) implies that $\left\|y x_{n}-Q_{K_{n} F_{n}}^{G \cup S G}\left(y x_{n}\right)\right\|_{2} \leq c_{1}+c_{2}+4 \varepsilon$. Since $\left\|\left[x_{n}, y\right]\right\|_{2} \rightarrow 0$, we can find $N$ such that $\left\|x_{n} y-Q_{K_{n} F_{n}}^{G \cup S}\left(x_{n} y\right)\right\|_{2} \leq c_{1}+c_{2}+5 \varepsilon$, for all $n \geq N$.

Since $x_{n} y \in \mathcal{U}(p(M \bar{\otimes} N) p)$, we get that

$$
\left\|Q_{K_{n} F_{n}}^{G \cup S G}\left(x_{n} y\right)\right\|_{2} \geq\|p\|_{2}-\left(c_{1}+c_{2}+5 \varepsilon\right) .
$$


Using that $\left\|P_{S}\left(x_{n}\right)\right\| \leq|S|$ we get that $\left\|x_{n} y-P_{S}\left(x_{n}\right) y_{n}\right\|_{2} \leq\left\|x_{n}-P_{S}\left(x_{n}\right)\right\|_{2}\|y\|+$ $\left\|P_{S}\left(x_{n}\right)\left|\left\||| y-y_{n}\right\|_{2} \leq c_{2}+\right| S \mid \frac{2 \varepsilon}{|S|}=c_{2}+2 \varepsilon\right.$. Combining this inequality with (5.c) yields that

$$
\left\|Q_{K_{n} F_{n}}^{G \cup S G}\left(P_{S}\left(x_{n}\right) y_{n}\right)\right\|_{2} \geq\|p\|_{2}-\left(c_{1}+2 c_{2}+7 \varepsilon\right) .
$$

Since $y_{n} \in \mathcal{H}_{G}$, Lemma 5.1 gives that $Q_{K_{n} F_{n}}^{G \cup S G}\left(P_{S}\left(x_{n}\right) y_{n}\right)=Q_{K_{n} F_{n}}^{G \cup S G}\left(P_{S}\left(x_{n}\right)\right) y_{n}$ and that $\left\|Q_{K_{n}}^{G \cup S G}\left(P_{S}\left(x_{n}\right)\right)\right\| \leq 2|S|$. Thus, since $\|y\| \leq 1$, for all $n \geq N$ we have that

$$
\begin{aligned}
\left\|Q_{K_{n} F_{n}}^{G \cup S G}\left(x_{n}\right)\right\|_{2} & \geq\left\|Q_{K_{n} F_{n}}^{G \cup S G}\left(P_{S}\left(x_{n}\right)\right)\right\|_{2}-c_{2} \\
& \geq\left\|Q_{K_{n} F_{n}}^{G \cup S G}\left(P_{S}\left(x_{n}\right)\right) y\right\|_{2}-c_{2} \\
& \geq\left\|Q_{K_{n} F_{n}}^{G \cup S G}\left(P_{S}\left(x_{n}\right)\right) y_{n}\right\|_{2}-\left\|Q_{K_{n} F_{n}}^{G \cup S G}\left(P_{S}\left(x_{n}\right)\right)\left(y_{n}-y\right)\right\|_{2}-c_{2} .
\end{aligned}
$$

Since $\left\|y_{n}-y\right\|_{2} \leq \frac{2 \varepsilon}{|S|}$, (5.d) implies that the last term is greater than or equal to $\|p\|_{2}-\left(c_{1}+2 c_{2}+7 \varepsilon\right)-2|S| \frac{2 \varepsilon}{|S|}-c_{2}=c$, as claimed.

To prove the theorem, assume by contradiction that $C \nprec_{M \bar{\otimes} N} B^{\Gamma \bar{\otimes}} N$. By using the claim for all $m \geq 1$ we will construct finite subsets $G_{m}$ and $\left\{K_{m, n}\right\}_{n \geq 1}$ of $\Gamma$ such that

(i) $K_{m, n}$ is disjoint from $G_{1}, \ldots, G_{m}, K_{1, n}, \ldots, K_{m-1, n}$, for all $n \geq 1$,

(ii) $\sup _{n \geq 1}\left|K_{m, n}\right|<\infty$ and

(iii) $\lim \inf _{n \rightarrow \infty}\left\|Q_{K_{m, n}}^{G_{m}}\left(x_{n}\right)\right\|_{2} \geq c$.

Before proving this statement let us show how it leads to a contradiction. First, (i) implies that for every $m \geq 1$, the projections $Q_{K_{1, n}}^{G_{1}}, Q_{K_{2, n}}^{G_{2}}, \ldots, Q_{K_{m, n}}^{G_{m}}$ are mutually orthogonal (since $Q_{F}^{G} Q_{F^{\prime}}^{G^{\prime}}=0$, whenever $F$ is disjoint from $G, F^{\prime}$ and $G^{\prime}$ ). Second, for every $m \geq 1$ we have that

$$
\|p\|_{2}^{2}=\liminf _{n \rightarrow \infty}\left\|x_{n}\right\|_{2}^{2} \geq \sum_{l=1}^{m} \liminf _{n \rightarrow \infty}\left\|Q_{K_{l, n}}^{G_{l}}\left(x_{n}\right)\right\|_{2}^{2} .
$$

By (iii) this implies that $\|p\|_{2}^{2} \geq m c^{2}$, for all $m \geq 1$, a contradiction.

So, we are left with proving the above statement. We proceed by using induction on $m$. For $m=1$, if we let $K_{1, n}=F_{n}$ and $G_{1}=\emptyset$, then the statement is true by the hypothesis. Next, assume that we have constructed $G_{l},\left\{K_{l, n}\right\}_{n \geq 1}$, for all $l \leq m-1$. Since $\sup _{n \geq 1}\left|K_{l, n}\right|<\infty$, for all $l$, and $\sup _{n \geq 1}\left|F_{n}\right|<\infty$ we get that

$$
\left.L=\sup _{n \geq 1, l \leq m-1} \mid\left(G_{l} \cup K_{l, n}\right) F_{n}^{-1}\right) \mid<\infty .
$$

Recall that $M \bar{\otimes} N=\left(B^{\Gamma \bar{\otimes}} N\right) \rtimes \Gamma$. Since $C \nprec_{M \bar{\otimes} N} B^{\Gamma \bar{\otimes}} N$, by Theorem 1.3.2 we can find $y \in \mathcal{U}(C)$ such that for every finite set $T \subset \Gamma$ of cardinality $|T| \leq L$, we have that

$$
\left\|P_{T}(y)\right\|_{2}=\left(\sum_{\gamma \in T}\left\|E_{B^{\Gamma} \bar{\otimes} N}\left(y u_{\gamma}^{*}\right)\right\|_{2}^{2}\right)^{\frac{1}{2}} \leq \frac{\varepsilon}{2|S|} .
$$

Now, let $K, G \subset \Gamma$ be finite sets such that $\left\|y-Q_{G}(y)\right\|_{2} \leq \frac{\varepsilon}{|S|}$ and $\| y-$ $P_{K}(y) \|_{2} \leq \frac{\varepsilon}{2|S|}$. For every $n \geq 1$, define $K_{n}=K \backslash\left(\bigcup_{l \leq m-1}\left(G_{l} \cup K_{l, n}\right) F_{n}^{-1}\right)$. Since $\left|K \backslash K_{n}\right| \leq L$, by (5.e) we deduce that $\left\|P_{K}(y)-P_{K_{n}}(y)\right\|_{2} \leq \frac{\varepsilon}{2|S|}$ and thus $\left\|y-P_{K_{n}}(y)\right\|_{2} \leq \frac{\varepsilon}{|S|}$, for all $n \geq 1$. Since $F_{n} \rightarrow \infty$, as $n \rightarrow \infty$, we can find $s \geq 1$ 
such that $K F_{n} \cap(G \cup S G)=\emptyset$, for all $n \geq s$. Thus, $K_{n} F_{n}$ is disjoint from $G \cup S G$, for all $n \geq s$.

Altogether, the above claim yields that

$$
\liminf _{n \rightarrow \infty}\left\|Q_{K_{n} F_{n}}^{G \cup S G}\left(x_{n}\right)\right\|_{2} \geq c .
$$

Finally, set $K_{m, n}=K_{n} F_{n}$, for all $n \geq s, K_{m, n}=\emptyset$, for all $n \leq s-1$, and $G_{m}=G \cup S G$. Then (5.f) can be rewritten as $\liminf _{n \rightarrow \infty}\left\|Q_{K_{m, n}}^{G_{m}}\left(x_{n}\right)\right\|_{2} \geq c$; hence (iii) is verified. Since $\left|K_{m, n}\right| \leq|K|\left|F_{n}\right|$ and $\sup _{n \geq 1}\left|F_{n}\right|<\infty$, we get that (ii) also holds true. Also, by definition it is clear that $K_{m, n}$ is disjoint from $G_{l}$ and $K_{l, n}$, for all $1 \leq l \leq m-1$. This proves the above statement and the theorem.

\section{A DiChOTOMY RESUlt FOR SUbALGEBRAS}

In this section, we combine the results of the last four sections to prove Theorem F (which we restate below as Theorem 6.2): if $D$ is an abelian subalgebra of $M=$ $B^{\Gamma} \rtimes \Gamma$ whose normalizer has a "large intersection" with $L(\Gamma)$, then a corner of $D$ embeds into either $B^{\Gamma}$ or $L(\Gamma)$. In the more general context, when $M$ is a tensor product of two factors associated with Bernoulli actions, we obtain:

Theorem 6.1. Assume the notation from 3.1, i.e. $M_{i}=B_{i}^{\Gamma_{i}} \rtimes_{\sigma_{i}} \Gamma_{i}, M=M_{1} \bar{\otimes} M_{2}$, $A=B_{1}^{\Gamma_{1}} \bar{\otimes} B_{2}^{\Gamma_{2}}$ and $\Gamma=\Gamma_{1} \times \Gamma_{2}$. Let $D \subset q M q$ be an abelian von Neumann subalgebra, for some projection $q \in L(\Gamma)$. Denote $\Lambda=\mathcal{N}_{q M q}(D) \cap \mathcal{U}(q L(\Gamma) q)$ and assume that $\Lambda^{\prime \prime} \nprec_{M} L\left(\Gamma_{1}\right) \otimes 1$ and $\Lambda^{\prime \prime} \nprec_{M} 1 \otimes L\left(\Gamma_{2}\right)$. Then one of the following holds true:

(1) $D^{\prime} \cap q M q$ is of type $I$ and there exist a unitary $u \in M$ and a projection $q_{0} \in A$ such that $u q_{0} u^{*}=q$ and $u\left(A q_{0}\right) u^{*} \subset D^{\prime} \cap q M q$.

(2) $D \prec_{M} M_{1} \bar{\otimes} L\left(\Gamma_{2}\right)$ or $D \prec_{M} L\left(\Gamma_{1}\right) \bar{\otimes} M_{2}$.

Recall that $\Lambda^{\prime \prime}$ denotes the von Neumann algebra generated by $\Lambda$ inside $q L(\Gamma) q$.

Proof. We first prove the theorem in the case $q=1$. Assume that (2) is false. We will show that (1) holds true. By using the hypothesis and Theorem 1.3.1 (see the comment following it) we can find a sequence $\left\{u_{n}\right\}_{n \geq 1} \subset \Lambda$ such that

$$
\left\|E_{L\left(\Gamma_{1}\right)}\left(a u_{n} b\right)\right\|_{2} \rightarrow 0 \text { and }\left\|E_{L\left(\Gamma_{2}\right)}\left(a u_{n} b\right)\right\|_{2} \rightarrow 0, \forall a, b \in L(\Gamma) .
$$

Next, we claim that

$$
\left(u_{n} x u_{n}^{*}\right)_{n} \in A^{\omega} \rtimes_{\sigma} \Gamma, \forall x \in D .
$$

To prove (6.b), let $\tilde{M}$ and $\left\{\theta_{t}\right\}_{t \in \mathbb{R}} \subset \operatorname{Aut}(\tilde{M})$ be defined as in the statement of Theorem 3.2. Since $\theta_{t \mid L(\Gamma)}=\operatorname{id}_{\mid L(\Gamma)}$ and $u_{n} \in L(\Gamma)$, we get that the $\theta_{t}$ converge uniformly to $\operatorname{id}_{\tilde{M}}$ on $\left\{u_{n} x u_{n}^{*}\right\}_{n \geq 1}$. As $D$ is abelian and $u_{n}$ normalizes $D$, we deduce that $\left(u_{n} x u_{n}^{*}\right)_{n} \in D^{\prime} \cap M^{\omega}$. Since (2) is assumed false, (6.b) follows from Theorem 3.2 .

Towards proving (1), let $C=D^{\prime} \cap M$. Since $D$ is abelian, we have that $C^{\prime} \cap M \subset$ $C$ or, equivalently, $C^{\prime} \cap M=\mathcal{Z}(C)$. The main part of this proof consists of showing that

$$
C p \prec_{M} B_{1}^{\Gamma_{1}} \bar{\otimes} M_{2}, \forall p \in \mathcal{P}(\mathcal{Z}(C)) .
$$

By symmetry, we will also get that $C p \prec_{M} M_{1} \bar{\otimes} B_{2}^{\Gamma_{2}}$. Finally, we will combine these two statements to first get that $C p \prec_{M} A$ and then derive (1). 
First, we reduce (6.c) to a weaker statement. By Lemma 1.3.3, it suffices to prove (6.c) for projections $p \in \mathcal{Z}(C)$ which commute with the normalizer of $C$. Since $\Lambda$ normalizes $C$, we get that $[p, \Lambda]=0$. Since $\Lambda^{\prime \prime} \nprec_{L(\Gamma)} L\left(\Gamma_{1}\right)$ and $\Lambda^{\prime \prime} \nprec_{L(\Gamma)} L\left(\Gamma_{2}\right)$, 45, Lemma 4.2 implies that $\Lambda^{\prime} \cap M \subset L(\Gamma)$, so in particular $p \in L(\Gamma)$.

Indeed, notice that if $B_{1} \simeq B_{2} \simeq L^{\infty}\left(X_{0}, \mu_{0}\right)$, then the action of $\Gamma$ on $A$ can be identified with the generalized Bernoulli action $\Gamma \curvearrowright\left(X_{0}, \mu_{0}\right)^{I}$, where $I=\Gamma_{1} \sqcup \Gamma_{2}$ and $\left(\gamma_{1}, \gamma_{2}\right) \cdot g_{1}=\gamma_{1} g_{1},\left(\gamma_{1}, \gamma_{2}\right) \cdot g_{2}=\gamma_{2} g_{2}$, for all $\gamma_{1}, g_{1} \in \Gamma_{1}$ and $\gamma_{2}, g_{2} \in \Gamma_{2}$. By applying [45, Lemma 4.2 to $I_{1}=\emptyset$, we get our assertion. For not necessarily isomorphic $B_{1}$ and $B_{2}$, it is not hard to adapt the proof of [45], Lemma 4.2 to prove our assertion.

Altogether, we get that it suffices to prove (6.c) for projections $p \in \Lambda^{\prime} \cap L(\Gamma) \cap$ $\mathcal{Z}(C)$.

For $n \geq 1$, let $v_{n}=u_{n} p \in L(\Gamma)$. Since (2) is false, we can find $x \in \mathcal{U}(D)$ such that $\left\|E_{M_{1} \bar{\otimes} L\left(\Gamma_{2}\right)}(x p)\right\|_{2} \leq \frac{1}{2}\|p\|_{2}$ and $\left\|E_{L\left(\Gamma_{1}\right) \bar{\otimes} M_{2}}(x p)\right\|_{2} \leq \frac{1}{2}\|p\|_{2}$. Since $p$ commutes with $u_{n} \in \Lambda$ and $x \in D$, we get that $v_{n}(x p) v_{n}^{*}=u_{n} x u_{n}^{*} p$. Since $\left(u_{n} x u_{n}^{*}\right)_{n} \in A^{\omega} \rtimes \Gamma$ and $\left\|u_{n} x u_{n}^{*} p\right\|_{2}=\|p\|_{2}$, for all $n$, by applying Theorem 4.1 we deduce that $\lim _{n \rightarrow \omega} h\left(v_{n}\right)>0$. By replacing $u_{n}$ with a subsequence, (6.a) and (6.b) are preserved while we may assume that there is $\delta>0$ such that

$$
h\left(v_{n}\right) \geq \delta, \forall n \geq 1 \text {. }
$$

For $n \geq 1$, let $\gamma_{n}=\left(\gamma_{n}^{1}, \gamma_{n}^{2}\right) \in \Gamma=\Gamma_{1} \times \Gamma_{2}$ such that $\left|\tau\left(v_{n} u_{\gamma_{n}}^{*}\right)\right|=h\left(v_{n}\right)$. We claim that $\gamma_{n}^{1} \rightarrow \infty$. If not, then $\gamma_{n_{k}}^{1}=\gamma_{1}$, for some increasing subsequence $\left\{n_{k}\right\}_{k \geq 1}$ of $\mathbb{N}$ and some $\gamma_{1} \in \Gamma_{1}$. But this would imply that

$$
\left.|| E_{L\left(\Gamma_{2}\right)}\left(u_{n_{k}} p\left(u_{\gamma_{1}}^{*} \otimes 1\right)\right)\right|_{2}=|| E_{L\left(\Gamma_{2}\right)}\left(v_{n_{k}}\left(u_{\gamma_{1}}^{*} \otimes 1\right)\right) \|_{2} \geq \tau\left(v_{n_{k}} u_{\gamma_{n_{k}}}^{*}\right) \mid \geq \delta, \quad \forall k \geq 1
$$

in contradiction with (6.a). Similarly, it follows that $\gamma_{n}^{2} \rightarrow \infty$.

Given finite subsets $F, S$ of $\Gamma_{1}$ and $T$ of $\Gamma_{2}$, we denote by:

- $\mathcal{K}_{F}$ the closed linear span of $\left\{\left(B_{1}^{F} \ominus \mathbb{C} 1\right) u_{\gamma_{1}} \otimes M_{2} \mid \gamma_{1} \in \Gamma_{1}\right\}$.

- $Q_{F}^{0}$ the orthogonal projection from $L^{2}(M)$ onto $\mathcal{K}_{F}$.

- $P_{S}$ the orthogonal projection onto the closed linear span of $\left\{B_{1}^{\Gamma_{1}} u_{\gamma_{1}} \otimes M_{2} \mid \gamma_{1} \in S\right\}$.

- $R_{T}$ the orthogonal projection onto the closed linear span of $\left\{M_{1} \otimes B_{2}^{\Gamma_{2}} u_{\gamma_{2}} \mid \gamma_{2} \in T\right\}$.

Claim 1. For every $x \in(M)_{1}$, every subset $F \subset \Gamma_{1}$ and all $n \geq 1$, we have that $\left\|v_{n} x v_{n}^{*}-Q_{\gamma_{n}^{1} F}^{0}\left(v_{n} x v_{n}^{*}\right)\right\|_{2} \leq\left\|x-Q_{F}^{0}(x)\right\|_{2}+\sqrt{\|p\|_{2}^{2}-\delta^{2}}$.

Proof of Claim 1. Recall that $\gamma_{n}=\left(\gamma_{n}^{1}, \gamma_{n}^{2}\right)$. Since $u_{\gamma_{n}} \mathcal{K}_{F}=\mathcal{K}_{\gamma_{n}^{1} F}, v_{n} \in L(\Gamma)$ and $\mathcal{K}_{F}$ is a right $L(\Gamma)$-module, we get that $u_{\gamma_{n}} Q_{F}^{0}(x) v_{n}^{*} \in \mathcal{K}_{\gamma_{n}^{1} F}$. Then, since ||$\tau\left(v_{n} u_{\gamma_{n}}^{*}\right) u_{\gamma_{n}} \|=\left|\tau\left(v_{n} u_{\gamma_{n}}^{*}\right)\right| \leq 1$, the triangle inequality gives that

$$
\begin{aligned}
& \left\|\left(\tau\left(v_{n} u_{\gamma_{n}}^{*}\right) u_{\gamma_{n}}\right) Q_{F}^{0}(x) v_{n}^{*}-v_{n} x v_{n}^{*}\right\|_{2} \\
& \quad \leq\left\|\left(\tau\left(v_{n} u_{\gamma_{n}}^{*}\right) u_{\gamma_{n}}\right)\left(Q_{F}^{0}(x)-x\right) v_{n}^{*}\right\|_{2}+\left\|\left(\tau\left(v_{n} u_{\gamma_{n}}^{*}\right) u_{\gamma_{n}}-v_{n}\right) x v_{n}^{*}\right\|_{2} \\
& \quad \leq\left\|Q_{F}^{0}(x)-x\right\|_{2}+\left\|\tau\left(v_{n} u_{\gamma_{n}}^{*}\right) u_{\gamma_{n}}-v_{n}\right\|_{2} \\
& \quad=\left\|Q_{F}^{0}(x)-x\right\|_{2}+\left(\left\|v_{n}\right\|_{2}^{2}-\left|\tau\left(v_{n} u_{\gamma_{n}}^{*}\right)\right|^{2}\right)^{\frac{1}{2}} .
\end{aligned}
$$

Together with (6.d) this implies the claim.

Since (2) is assumed false we can find $x \in \mathcal{U}(D)$ such that $\left\|E_{L\left(\Gamma_{1}\right) \otimes M_{2}}(x)\right\|_{2}<$ $\frac{1}{2}\left(\|p\|_{2}-\sqrt{\|p\|_{2}^{2}-\delta^{2}}\right)$. If we enumerate $\Gamma_{1}=\left\{g_{i}\right\}_{i \geq 1}$ and let $F_{n}=\left\{g_{i}\right\}_{i=1}^{n}$, then 
$\left\|E_{L\left(\Gamma_{1}\right) \bar{\otimes} M_{2}}(x)-\left(x-Q_{F_{n}}^{0}(x)\right)\right\|_{2} \rightarrow 0$. Thus, we can find a finite subset $F \subset \Gamma_{1}$ such that $\left\|x-Q_{F}^{0}(x)\right\|_{2}<\frac{1}{2}\left(\|p\|_{2}-\sqrt{\|p\|_{2}^{2}-\delta^{2}}\right)$.

If we let $x_{n}=v_{n} x v_{n}^{*}$, by Claim 1 we get that

$$
\left\|x_{n}-Q_{\gamma_{n}^{1} F}^{0}\left(x_{n}\right)\right\|_{2}<\frac{1}{2}\left(\|p\|_{2}+\sqrt{\|p\|_{2}^{2}-\delta^{2}}\right), \forall n \geq 1 .
$$

Since $p$ commutes with $u_{n}, D$ and $u_{n}$ normalizes $D$, we have that $x_{n}=v_{n} x v_{n}^{*}=$ $\left(u_{n} x u_{n}^{*}\right) p \in \mathcal{U}(D p)$, for all $n \geq 1$. Thus, we deduce that $\left(x_{n}\right)_{n} \in \mathcal{U}\left((C p)^{\prime} \cap(p M p)^{\omega}\right)$. By (6.a) we have that $\left(x_{n}\right)_{n}=\left(u_{n} x u_{n}^{*} p\right)_{n} \in A^{\omega} \rtimes_{\sigma} \Gamma$. Hence, we can find a finite subset $S \subset \Gamma_{1}$ such that $\lim \sup _{n \rightarrow \infty}\left\|x_{n}-P_{S}\left(x_{n}\right)\right\|_{2}<\frac{1}{6}\left(\|p\|_{2}-\sqrt{\|p\|_{2}^{2}-\delta^{2}}\right)$. By combining this inequality with (6.e) we get that

$$
\limsup _{n \rightarrow \infty}\left(\left\|x_{n}-Q_{\gamma_{n}^{1} F}^{0}\left(x_{n}\right)\right\|_{2}+3\left\|x_{n}-P_{S}\left(x_{n}\right)\right\|_{2}\right)<\|p\|_{2} .
$$

Altogether, Theorem 5.2 yields that $C p \prec_{M} B_{1}^{\Gamma_{1}} \bar{\otimes} M_{2}$. This finishes the proof of (6.c).

Claim 2. $C p \prec_{M} A=B_{1}^{\Gamma_{1}} \bar{\otimes} B_{2}^{\Gamma_{2}}$, for all $p \in \mathcal{P}(\mathcal{Z}(C))$.

Proof of Claim 2. By (6.c), we have that $C p \prec_{M} B_{1}^{\Gamma_{1}} \bar{\otimes} M_{2}$. Since $C^{\prime} \cap M=\mathcal{Z}(C)$, by Theorem 1.3.1 we can find non-zero projections $p_{0} \in C p$ and $q \in B_{1}^{\Gamma_{1}} \bar{\otimes} M_{2}$, a $*$-homomorphism $\psi: p_{0} C p_{0} \rightarrow q\left(B_{1}^{\Gamma_{1}} \bar{\otimes} M_{2}\right) q$ and a non-zero partial isometry $v \in q M p_{0}$ such that $v^{*} v=p_{0}$ and $\psi(x) v=v x$, for all $x \in p_{0} C p_{0}$. Thus, we have that

$$
x=v^{*} \psi(x) v, \forall x \in p_{0} C p_{0} .
$$

Now, let $z \leq p$ be the central support of $p_{0}$ in $C$. By symmetry, the proof of (6.c) implies that $C z \prec_{M} M_{1} \bar{\otimes} B_{2}^{\Gamma_{2}}$. By reasoning as in the previous paragraph, we can find non-zero projections $p_{1} \in C z$ and $r \in M_{1} \bar{\otimes} B_{2}^{\Gamma_{2}}$, a $*$-homomorphism $\rho: p_{1} C p_{1} \rightarrow r\left(M_{1} \bar{\otimes} B_{2}^{\Gamma_{2}}\right) r$ and a non-zero partial isometry $w \in r M p_{1}$ such that

$$
x=w^{*} \rho(x) w, \forall x \in p_{1} C p_{1} .
$$

Since $p_{0}$ and $p_{1}$ admit equivalent, non-zero subprojections, we can assume that (6.f) and (6.g) hold true for $p_{0}=p_{1} \leq p$.

By Kaplansky's density theorem we can find finite subsets $S \subset \Gamma_{1}, T \subset \Gamma_{2}$ and $v^{\prime}, w^{\prime} \in(M)_{1}$ satisfying $\left\|v^{\prime}-v\right\|_{2},\left\|w^{\prime}-w\right\|_{2} \leq \frac{\left\|p_{0}\right\|_{2}}{6}$ and $v^{\prime}=P_{S}\left(v^{\prime}\right), w^{\prime}=R_{T}\left(w^{\prime}\right)$. Using (6.f), (6.g) and the triangle inequality it follows that

$$
\left\|x-P_{S^{-1} S}(x)\right\|_{2},\left\|x-R_{T^{-1} T}(x)\right\|_{2} \leq \frac{\left\|p_{0}\right\|_{2}}{3}, \forall x \in \mathcal{U}\left(p_{0} C p_{0}\right) .
$$

Finally, (6.h) implies that $\left\|x-\left(P_{S^{-1} S} \circ R_{T^{-1} T}\right)(x)\right\|_{2} \leq \frac{2\left\|p_{0}\right\|_{2}}{3}$, or equivalently, that $\left\|\left(P_{S^{-1} S} \circ R_{T^{-1} T}\right)(x)\right\|_{2} \geq \frac{\sqrt{5}}{3}\left\|p_{0}\right\|_{2}$, for all $x \in \mathcal{U}\left(p_{0} C p_{0}\right)$.

Since $P_{S^{-1} S} \circ R_{T^{-1} T}$ is precisely the orthogonal projection on the closed linear span of $\left\{A u_{\gamma} \mid \gamma \in S^{-1} S \times T^{-1} T\right\}$, by Theorem 1.3 .1 we get that $p_{0} C p_{0} \prec_{M} A$. Thus, $C p \prec_{M} A$.

Claim 3. $C$ is a type I algebra and there exists a unitary $u \in M$ such that $u A u^{*} \subset$ C. 
Proof of Claim 3. The first assertion follows from Claim 2. For the second assertion, let $C_{0}$ be a maximal abelian subalgebra of $C$. Then $C_{0}$ is maximal abelian in $M$. Indeed, $C_{0}$ contains the center of $C$; hence it contains $D$ and therefore $C_{0}^{\prime} \cap M \subset D^{\prime} \cap M=C$.

Next, let $p \in \mathcal{P}(\mathcal{Z}(C))$. By Claim $2, C p \prec_{M} A$ and therefore $C_{0} p \prec_{M} A$. Since $C_{0} p \subset p M p$ and $A \subset M$ are maximal abelian, by [35, Theorem A.1 (see also [44, Lemma C.3) we can find a non-zero projection $p^{\prime} \in C_{0} p$ and a unitary $v \in M$ such that $C_{0} p^{\prime} \subset v A v^{*}$. Let $z$ be the central support of $p^{\prime}$ in $C$. Since $C$ is of type I, we have that $C_{0}$ is regular in $C$, i.e. $\mathcal{N}_{C}\left(C_{0}\right)^{\prime \prime}=C$. It follows that we can find projections $p_{1}, p_{2}, \ldots \in C_{0} p^{\prime}$ and $u_{1}, u_{2}, \ldots \in \mathcal{N}_{C}\left(C_{0}\right)$ such that $z=\sum_{i \geq 1} u_{i} p_{i} u_{i}^{*}$.

Further, since $u_{i} \in \mathcal{N}_{C}\left(C_{0}\right)$ we get that $C_{0}\left(u_{i} p_{i} u_{i}^{*}\right)=u_{i}\left(C_{0} p_{i}\right) u_{i}^{*} \subset\left(u_{i} v\right) A\left(u_{i} v\right)^{*}$, for all $i$. Since $A$ is regular in $M$ and $M$ is a $\mathrm{II}_{1}$ factor, it follows that we can find a unitary $w \in M$ such that $C_{0} z \subset w A w^{*}$. We have altogether shown that for every non-zero projection $p \in \mathcal{Z}(C)$, there is a non-zero projection $z \in \mathcal{Z}(C)$ with $z \leq p$ such that $C_{0} z$ can be unitarily conjugated inside $A$.

Finally, let $\mathcal{S}$ be the set of families $\left\{p_{i}\right\}_{i \in I} \subset \mathcal{P}(\mathcal{Z}(C))$ of mutually orthogonal projections with the property that $C_{0} p_{i} \subset u_{i} A u_{i}^{*}$, for some $u_{i} \in \mathcal{U}(M)$, for all $i \in I$. By the above we get that if $\left\{p_{i}\right\}_{i \in I}$ is a maximal element in $\mathcal{S}$ with respect to inclusion, then $\sum_{i \in I} p_{i}=1$. Using again the fact that $A$ is regular in $M$, we deduce that $C_{0}$ can be unitarily conjugated inside $A$. Since $C_{0}$ is maximal abelian, we get that $C_{0}=u A u^{*}$, for some $u \in \mathcal{U}(M)$. This ends the proof of Claim 3 and of the case $q=1$.

In general, let $D \subset q M q$ be a subalgebra as in the hypothesis and assume that (2) is false. Then (1) follows true by repeating the above proof once we show that (6.b) holds true in this context, i.e. $\left(u_{n} x u_{n}^{*}\right)_{n} \in A^{\omega} \rtimes_{\sigma} \Gamma$, for all $x \in D$ and any sequence $\left\{u_{n}\right\}_{n \geq 1} \subset \Lambda$. To see this, let $D_{0} \subset(1-q) M(1-q)$ be a unital, abelian von Neumann subalgebra such that $D_{0} \nprec_{M} M_{1} \bar{\otimes} L\left(\Gamma_{2}\right)$ and $D_{0} \nprec_{M} L\left(\Gamma_{1}\right) \bar{\otimes} M_{2}$. Then $D_{1}=D \oplus D_{0}$ has the same property. Since $D_{1} \subset M$ is unital and the $\theta_{t}$ converge uniformly to the identity on $\left(u_{n} x u_{n}^{*}\right)_{n} \in D_{1}^{\prime} \cap M^{\omega}$, our claim follows from Theorem 3.2.

Note that all of our results concerning tensor products of $\mathrm{II}_{1}$ factors associated with Bernoulli actions (Theorems 3.3, 4.1, 6.1) have straightforward counterparts for single such $\mathrm{II}_{1}$ factors. In this case, Theorem 6.1 reads as follows:

Theorem 6.2. Let $\Gamma$ be a countable group, $B$ be an abelian von Neumann algebra and set $M=B^{\Gamma} \rtimes_{\sigma} \Gamma, A=B^{\Gamma}$. Let $q \in L(\Gamma)$ be a projection and $D \subset q M q$ be a unital abelian von Neumann subalgebra. Suppose that the group of unitary elements $u \in q L(\Gamma) q$ that normalize $D$ generates a diffuse von Neumann algebra. Then either:

(1) $D^{\prime} \cap q M q$ is of type $I$ and there exist a unitary $u \in M$ and a projection $q_{0} \in A$ such that $u q_{0} u^{*}=q$ and $u\left(A q_{0}\right) u^{*} \subset D^{\prime} \cap q M q$ or

(2) $D \prec_{M} L(\Gamma)$.

We end this section by noticing that Theorem 6.2 can be used to give a proof of Popa's conjugacy criterion for Bernoulli actions.

Theorem 6.3 (Popa, 34, Theorem 0.7). Let $\Gamma$ be a countable ICC group, $B$ be a non-trivial abelian von Neumann algebra. Denote $M=B^{\Gamma} \rtimes \Gamma, A=B^{\Gamma}$ and let $q \in L(\Gamma)$ be a projection. Let $\rho: \Lambda \rightarrow \operatorname{Aut}(C)$ be a free ergodic action of a 
countable group $\Lambda$ on an abelian von Neumann algebra $C$. Denote $N=C \rtimes \Lambda$. Let $\theta: N \rightarrow q M q$ be a $*$-isomorphism. Assume that $\theta(L(\Lambda)) \subset q L(\Gamma) q$. Then $q=1$ and there exist a unitary $u \in M$, a character $\eta$ of $\Lambda$, a group isomorphism $\delta: \Lambda \rightarrow \Gamma$ such that $\theta(C)=u A u^{*}$ and $\theta\left(v_{\lambda}\right)=\eta(\lambda) u u_{\delta(\lambda)} u^{*}$, for all $\lambda \in \Lambda$.

Proof. Denote $D=\theta(C)$. Since $\sigma$ is mixing and $D$ is regular in $q M q$, 33, Theorem 3.1 implies that $D \nprec_{M} L(\Gamma)$. Since $D$ is normalized by $\left\{\theta\left(v_{\lambda}\right)\right\}_{\lambda \in \Lambda} \subset q L(\Gamma) q$ and $D$ is maximal abelian in $q M q$, Theorem 6.2 implies that there exists a unitary $u \in M$ and a projection $q_{0} \in A$ such that $u q_{0} u^{*}=q$ and $D=u\left(A q_{0}\right) u^{*}$. The conclusion now follows from 34, Theorem 5.2.

\section{POPA's CONJUGACY CRITERION FOR ACTIONS}

In the proof of the strong rigidity results we will need the following version of Popa's conjugacy criterion for actions.

Theorem 7.1 ([34]). Let $\sigma: \Gamma \rightarrow \operatorname{Aut}(A)$ and $\beta: \Lambda \rightarrow \operatorname{Aut}(B)$ be two free, ergodic actions of two countable groups $\Gamma$ and $\Lambda$ on two abelian von Neumann algebras $A$ and B. Assume that $\beta$ is weakly mixing. Denote $M=A \rtimes_{\sigma} \Gamma$ and $N=B \rtimes_{\beta} \Lambda$. Let $\left\{u_{\gamma}\right\}_{\gamma \in \Gamma} \subset M$ and $\left\{v_{\lambda}\right\}_{\lambda \in \Lambda} \subset N$ be the canonical unitaries implementing $\sigma$ and $\beta$. Let $p \in L(\Gamma)$ be a non-zero projection and assume that $N$ is embedded (unitaly) inside $p M p$ such that $L(\Lambda) \subset p L(\Gamma) p$. Suppose that there exists a partial isometry $v \in M$ such that $v^{*} v=p, v v^{*}=q \in A$ and $v B v^{*}=A q$.

Then we can find maps $h: \Lambda \rightarrow S^{1}, \delta: \Lambda \rightarrow \Gamma$ and a unitary $z \in L(\Gamma)$ such that $v_{\lambda}=h(\lambda)\left(z u_{\delta(\lambda)} z^{*}\right) p$ and $\left[z u_{\delta(\lambda)} z^{*}, p\right]=0$, for all $\lambda \in \Lambda$. Let $K=\{\gamma \in$ $\left.\Gamma \mid\left(z u_{\gamma} z^{*}\right) p \in \mathbb{C} p\right\}$ and $\Gamma^{\prime}=\{\delta(\lambda) k \mid \lambda \in \Lambda, k \in K\}$. Then $K, \Gamma^{\prime}$ are subgroups of $\Gamma$, $|K|<\infty$ and $\Gamma^{\prime}$ normalizes $K$.

Moreover, we have that:

- If $\Gamma$ is torsion free, then $h$ is a character and $\delta$ is a homomorphism. If in addition the restriction of $\sigma$ to $\delta(\Lambda)$ is ergodic, then $p=q=1$ and $B=z A z^{*}$.

- In general, if the restriction of $\sigma$ to $\Gamma^{\prime}$ is weakly mixing, then $z^{*} p z \in L(K) \cap$ $\mathcal{Z}\left(L\left(\Gamma^{\prime}\right)\right), z^{*} p z=|K|^{-1} \sum_{k \in K} \chi(k) u_{k}$, for some character $\chi$ of $K$, and $B=$ $\left(z A^{K} z^{*}\right) p$, where $A^{K}=\left\{a \in A \mid \sigma_{k}(a)=a, \forall k \in K\right\}$. In particular, $\tau(p)^{-1}=|K|$ is an integer.

The statement of this result is very close to that of Theorem 5.2 in [34. However, rather than assuming that $\sigma$ is mixing, as in [34, we only require that $\sigma_{\mid \delta(\Lambda)}$ is ergodic (in the torsion free case) and weakly mixing (in general). This generalization will be essential for us, as we will later apply Theorem 7.1 to the action $\sigma$ defined in 3.1, for which the "global" condition $\sigma$ is mixing fails. On the other hand, since $\sigma$ is a concrete action (i.e., a product of two Bernoulli actions) it will be easy to verify the "local" condition that $\sigma_{\mid \delta(\Lambda)}$ is weakly mixing.

Proof. In the beginning of the proof we repeat part of Vaes' proof of Popa's criterion (see 44, Proposition 9.3) which streamlines the original argument from [34.

Identify $A$ with $L^{\infty}(X, \mu)$, where $(X, \mu)$ is a probability space. Let $Y \subset X$ be a Borel set such that $q=1_{Y}$. Note that $v v_{\lambda} v^{*} \in \mathcal{U}(q M q)$ normalizes $A q \cong L^{\infty}(Y)$, for all $\lambda \in \Lambda$, and denote by $\beta^{\prime}$ the associated action of $\Lambda$ on $Y$. Since $\beta^{\prime}$ is isomorphic to $\beta$, we have that $\beta^{\prime}$ is weakly mixing. We consider the canonical embedding $\eta: L^{\infty}(X) \rtimes_{\sigma} \Gamma \subset L^{\infty}\left(X, \ell^{2}(\Gamma)\right)$ given by $\eta\left(a u_{\gamma}\right)(x)=a(x) u_{\gamma}$, for all 
$a \in L^{\infty}(X), x \in X$ and $\gamma \in \Gamma$, as well as the embedding $L(\Gamma) \subset \ell^{2}(\Gamma)$. Also, we view $S^{1} \times \Gamma$ as a subgroup of $\mathcal{U}(L(\Gamma))$, in the natural way.

Let $w=\tau(p)^{\frac{1}{2}} \eta(v) \in L^{\infty}\left(Y, \ell^{2}(\Gamma)\right)$. Since $\beta$ is weakly mixing, 34, Lemma 4.4(i) implies that $B=v^{*} A v$ is orthogonal to $L(\Gamma)$. The same argument as in the proofs of [34, Lemma 6.1 and [44, Proposition 9.3 shows that every essential value $w_{0}$ of the function $w: Y \rightarrow \ell^{2}(\Gamma)$ lies in $L(\Gamma)$ and satisfies $w_{0}^{*} w_{0}=p$. Set $v^{\prime}=v w_{0}^{*}, p^{\prime}=v^{\prime *} v^{\prime}=w_{0} p w_{0}^{*}=w_{0} w_{0}^{*}, w^{\prime}=\tau(p)^{\frac{1}{2}} \eta\left(v^{\prime}\right)$ and notice that $p^{\prime}$ is an essential value of $w^{\prime}$. By replacing $v$ with $v^{\prime}, p$ with $p^{\prime}, v_{\lambda}$ with $w_{0} v_{\lambda} w_{0}^{*}$ and $B$ with $w_{0} B w_{0}^{*}$, we may assume that $p$ is an essential value of $w$, while the hypothesis is still satisfied. For the "new" $v, p, v_{\lambda}$ we will prove the conclusion for $z=1$, which clearly finishes the proof.

Let $\lambda \in \Lambda$. Since $v v_{\lambda} v^{*} \in \mathcal{N}_{q M q}(A q)$, we can find $a_{\lambda} \in \mathcal{U}\left(L^{\infty}(Y)\right)$ such that $v v_{\lambda} v^{*}=a_{\lambda} \sum_{\gamma \in \Gamma} 1_{\left\{y \mid \lambda^{-1} y=\gamma^{-1} y\right\}} u_{\gamma}$, where we denote $\lambda y=\beta_{\lambda}^{\prime}(y)$, for every $y \in Y$.

Let $\omega=\sum_{g \in \Gamma} b_{g} u_{g} \in M$, where $b_{g} \in A$. Then

$$
\left(v v_{\lambda} v^{*}\right) \omega=\sum_{\gamma, g \in \Gamma} a_{\lambda} 1_{\left\{y \mid \lambda^{-1} y=\gamma^{-1} y\right\}}\left(b_{g} \circ \gamma^{-1}\right) u_{\gamma g} .
$$

Thus, if we let $c(\lambda, y)=a_{\lambda}(y) u_{\gamma} \in S^{1} \times \Gamma \subset \mathcal{U}(L(\Gamma))$, where $\gamma \in \Gamma$ is the unique element such that $\gamma^{-1} y=\lambda^{-1} y$, then

$$
\begin{gathered}
\eta\left(\left(v v_{\lambda} v^{*}\right) \omega\right)(y)=\sum_{\gamma, g \in \Gamma} a_{\lambda}(y) 1_{\left\{y \mid \lambda^{-1} y=\gamma^{-1} y\right\}}(y) b_{g}\left(\gamma^{-1} y\right) u_{\gamma g} \\
=\left(a_{\lambda}(y) u_{\gamma}\right)\left(\sum_{g \in \Gamma} b_{g}\left(\lambda^{-1} y\right) u_{g}\right)=c(\lambda, y) \eta(\omega)\left(\lambda^{-1} y\right), \text { for almost all } y \in Y .
\end{gathered}
$$

Since $\left(v v_{\lambda} v^{*}\right) v=v v_{\lambda}$ and $v_{\lambda} \in L(\Gamma)$, by applying $\eta$ and using (7.a), we get that

$$
c(\lambda, y) w\left(\lambda^{-1} y\right)=w(y) v_{\lambda}, \quad \text { for almost all } y \in Y .
$$

Next, we use an argument due to Popa (see the proofs of [37], Proposition 3.5 and 44], Lemma 4.8) to conclude the first assertion of the theorem. If $\varepsilon>0$, then, since $p$ is an essential value of $w$, the set $W=\left\{y \in Y \mid\|w(y)-p\|_{2} \leq \varepsilon\right\}$ has positive measure. Fix $\lambda \in \Lambda$. Since $\beta^{\prime}$ is weakly mixing, we can find $g \in \Lambda$ such that $\mu(g W \cap W)>0$ and $\mu\left(\left(g \lambda^{-1}\right) W \cap W\right)>0$.

Let $y \in g W \cap W$. Then $\|w(y)-p\|_{2},\left\|w\left(g^{-1} y\right)-p\right\|_{2} \leq \varepsilon$ and by using (7.b) we deduce that

$$
\begin{aligned}
\left\|v_{g}-c(g, y) p\right\|_{2} & =\left\|p v_{g}-c(g, y) p\right\|_{2} \\
& =\left\|(p-w(y)) v_{g}-c(g, y)\left(p-w\left(g^{-1} y\right)\right)\right\|_{2} \leq 2 \varepsilon .
\end{aligned}
$$

Similarly, if $z \in\left(g \lambda^{-1}\right) W \cap W$, then $\left\|v_{g \lambda^{-1}}-c\left(g \lambda^{-1}, z\right) p\right\|_{2} \leq 2 \varepsilon$. Since $v_{\lambda}=$ $\left(v_{g \lambda^{-1}}\right)^{*} v_{g}$, the combination of the last two inequalities yields

$$
\left\|v_{\lambda}-p c\left(g \lambda^{-1}, z\right)^{*} c(g, y) p\right\|=\left\|v_{g \lambda^{-1}}^{*} v_{g}-\left(c\left(g \lambda^{-1}, z\right) p\right)^{*} c(g, y) p\right\|_{2} \leq 4 \varepsilon .
$$

Therefore, since $c\left(g \lambda^{-1}, z\right)^{*} c(g, y) \in S^{1} \times \Gamma$ and $\varepsilon>0$ is arbitrary, we can find two sequences $\left\{t_{n}\right\}_{n \geq 1} \subset S^{1}$ and $\left\{\gamma_{n}\right\}_{n \geq 1} \subset \Gamma$ such that $\lim _{n \rightarrow \infty}\left\|v_{\lambda}-p\left(t_{n} u_{\gamma_{n}}\right) p\right\|_{2}=0$. By passing to a subsequence we can assume that $t_{n} \rightarrow t \in S^{1}$ and either $\gamma_{n} \rightarrow \gamma \in \Gamma$ or $\gamma_{n} \rightarrow \infty$, as $n \rightarrow \infty$. If $\gamma_{n} \rightarrow \infty$, then $t_{n} u_{\gamma_{n}}$ converges weakly to 0 , which would imply that $v_{\lambda}=0$, a contradiction. Thus, we get that $\gamma_{n} \rightarrow \gamma \in \Gamma$; hence 
$v_{\lambda}=t p u_{\gamma} p$. Since $v_{\lambda} \in \mathcal{U}(p M p)$, we must have that $u_{\gamma}$ commutes with $p$. We altogether deduce that there exist maps $h: \Lambda \rightarrow S^{1}$ and $\delta: \Lambda \rightarrow \Gamma$ such that

$$
\left[u_{\delta(\lambda)}, p\right]=0 \text { and } v_{\lambda}=h(\lambda) u_{\delta(\lambda)} p, \forall \lambda \in \Lambda .
$$

As in the hypothesis, denote $K=\left\{\gamma \in \Gamma \mid u_{\gamma} p \in \mathbb{C} p\right\}$ and $\Gamma^{\prime}=\{\delta(\lambda) k \mid \lambda \in \Lambda, k \in$ $K\}$. Then $K$ is finite and (7.c) implies that $\Gamma^{\prime}$ is a subgroup of $\Gamma$ which contains $K$ as a normal subgroup. Notice also that the map $\Lambda \ni \lambda \rightarrow \delta(\lambda) K \in \Gamma^{\prime} / K$ is a group isomorphism.

We can now prove the moreover assertions.

- If $\Gamma$ is torsion free, then $K=\{e\}$ and (7.c) implies that $\delta$ is a homomorphism and $h$ is a character.

Further, assume that $\sigma_{\mid \delta(\Lambda)}$ is ergodic and let $\varepsilon>0$. Let $W_{\varepsilon}$ be the set of $y \in Y$ for which there exists $u \in S^{1} \times \Gamma \subset \mathcal{U}(L(\Gamma))$ such that $\|w(y)-u p\|_{2} \leq \varepsilon$. Since $p$ is an essential value of $w$, we have that $\mu\left(W_{\varepsilon}\right)>0$. On the other hand, (7.b) and (7.c) imply that $W_{\varepsilon}$ is $\Lambda$-invariant. Indeed, if $y \in Y, \lambda \in \Lambda$ and $u \in S^{1} \times \Gamma$, then $u^{\prime}=h(\lambda) c(\lambda, y)^{*} u u_{\delta(\lambda)} \in S^{1} \times \Gamma$ satisfies

$$
\begin{aligned}
\left\|w\left(\lambda^{-1} y\right)-u^{\prime} p\right\|_{2} & =\left\|c(\lambda, y)^{*} w(y) v_{\lambda}-h(\lambda) c(\lambda, y)^{*} u u_{\delta(\lambda)} p\right\| \\
& =\left\|c(\lambda, y)^{*} w(y) v_{\lambda}-c(\lambda, y)^{*} u p v_{\lambda}\right\|_{2}=\|w(y)-u p\|_{2} .
\end{aligned}
$$

Since $\beta^{\prime}$ is ergodic, we get that $W_{\varepsilon}=Y$. By reasoning as above we conclude that there exists a measurable map $u: Y \rightarrow S^{1} \times \Gamma$ such that $w(y)=u(y) p$, for a.e. $y \in Y$.

But then (7.b) can be rewritten as $c(\lambda, y) u\left(\lambda^{-1} y\right) p=h(\lambda) u(y) u_{\delta(\lambda)} p$, for all $\lambda \in \Lambda$ and for almost all $y \in Y$. Since $c(\lambda, y) u\left(\lambda^{-1} y\right), h(\lambda) u(y) u_{\delta(\lambda)} \in S^{1} \times \Gamma$, by using the fact that $K=\{e\}$, we get that

$$
c(\lambda, y) u\left(\lambda^{-1} y\right)=h(\lambda) u(y) u_{\delta(\lambda)}, \forall \lambda \in \Lambda \text { and for almost all } y \in Y .
$$

Finally, let $\omega \in L^{2}\left(A \rtimes_{\sigma} \Gamma\right) \cong L^{2}\left(X, \ell^{2}(\Gamma)\right)$ be defined by $\omega(x)=u(x)$, if $x \in Y$ and $\omega(x)=0$, otherwise. By using (7.a) and (7.d) we get that $\left(v v_{\lambda} v^{*}\right) \omega=$ $h(\lambda) \omega u_{\delta(\lambda)}$, for all $\lambda \in \Lambda$.

Since $\omega=q \omega$ and $v v_{\lambda} v^{*} \in \mathcal{U}(q M q)$, we further derive that

$$
\omega^{*} \omega=u_{\delta(\lambda)}\left(\omega^{*} \omega\right) u_{\delta(\lambda)}^{*}, \forall \lambda \in \Lambda .
$$

By the definition of $\omega$, we can find $a \in \mathcal{U}(A)$ and a measurable partition $\left\{A_{\gamma}\right\}_{\gamma \in \Gamma}$ of $Y$ such that $\omega=a \sum_{\gamma \in \Gamma} 1_{A_{\gamma}} u_{\gamma}$. Hence, we have that $\omega^{*} \omega=\sum_{\gamma \in \Gamma} u_{\gamma}^{*} 1_{A_{\gamma}} u_{\gamma}=$ $\sum_{\gamma \in \Gamma} 1_{\gamma^{-1} A_{\gamma}} \in A$. Since $\sigma_{\mid \delta(\Lambda)}$ is ergodic, by (7.e) we deduce that $\sum_{\gamma \in \Gamma} 1_{\gamma^{-1} A_{\gamma}} \in$ $\mathbb{C} 1$. This clearly implies that $Y=X$; hence $p=q=1$. Also, we get that $w(y)=u(y) \in S^{1} \times \Gamma$, for almost all $y \in X$; thus $v \in \mathcal{N}_{M}(A)$. This shows that $B=A$, which concludes the proof of the torsion free case.

- The proof of the general case is an adaptation of arguments from [34, Section 6. Assume that $\sigma_{\mid \Gamma^{\prime}}$ is weakly mixing. Since $p$ is an essential value for $w=\tau(p)^{\frac{1}{2}} \eta(v)$, we can find a decreasing sequence of non-zero projections $\left\{q_{n}\right\}_{n \geq 1} \subset A q$ such that

$$
\left\|q^{\prime} v-\tau(p)^{-\frac{1}{2}} q^{\prime} p\right\|_{2} \leq 2^{-n-1}\left\|q^{\prime}\right\|_{2}, \forall q^{\prime} \in \mathcal{P}\left(A q_{n}\right)
$$

for every $n \geq 1$ (see [34, Lemma 6.1).

Let us introduce some notation. Fix $n \geq 1$ and $\lambda \in \Lambda$. Since $p, v_{\lambda} \in L(\Gamma)$ we can decompose $p=\sum_{\gamma \in \Gamma} c_{\gamma} u_{\gamma}$ and $v_{\lambda}=\sum_{\gamma \in \Gamma} c_{\gamma}^{\lambda} u_{\gamma}$, with $c_{\gamma}, c_{\gamma}^{\lambda} \in \mathbb{C}$, for all 
$\gamma \in \Gamma$. Thus, we have that $q_{n} v_{\lambda} q_{n}=\sum_{\gamma \in \Gamma} c_{\gamma}^{\lambda} \sigma_{\gamma}\left(q_{n}\right) q_{n} u_{\gamma}$. Also, we decompose $q_{n} v v_{\lambda} v^{*} q_{n}=\sum_{\gamma \in \Gamma} a_{\gamma}^{\lambda, n} u_{\gamma}$, with $a_{\gamma}^{\lambda, n} \in A q$.

Let $F_{n}^{\lambda}=\left\{\gamma \in \Gamma \mid a_{\gamma}^{\lambda, n} \neq 0\right\}$. By using (7.f), 34, Lemma 6.3 gives that

$$
\left|a_{\gamma}^{\lambda, n}\right|=\sigma_{\gamma}\left(q_{n}\right) q_{n} \text { and }\left|1-\tau(p)^{-1}\right| c_{\gamma}^{\lambda}|| \leq 2^{-n}, \forall \gamma \in F_{n}^{\lambda} .
$$

Note that since $v_{\lambda}=h(\lambda) u_{\delta(\lambda)} p$ and $|h(\lambda)|=1$, we get that

$$
\left|c_{\gamma}^{\lambda}\right|=\left|c_{\delta(\lambda)^{-1} \gamma}\right|, \forall \gamma \in \Gamma, \lambda \in \Lambda \text {. }
$$

Next, we prove the following:

Claim. $\tau(p)^{-1} \in \mathbb{N}$ and $\left|c_{\gamma}\right| \in\{0, \tau(p)\}$, for all $\gamma \in \Gamma$.

Proof of Claim. Let $n \geq 1$ and $\varepsilon>0$. Set $F=\left\{\gamma \in \Gamma|| c_{\gamma} \mid \geq \frac{\tau(p)}{2}\right\}$. By (7.g), if $\gamma \in F_{n}^{\lambda}$, then $\left|c_{\gamma}^{\lambda}\right| \geq \frac{\tau(p)}{2}$. Using (7.h) this shows that $\delta(\lambda)^{-1} \gamma \in F$. In other words, $F_{n}^{\lambda} \subset \delta(\lambda) F$, for all $\lambda \in \Lambda$.

Now, since $v v_{\lambda} v^{*}$ normalizes $A q$ we get that $a_{\gamma}^{\lambda, n}$ are partial isometries in $A q$. Thus, using the definitions of $F_{n}^{\lambda}, a_{\gamma}^{\lambda, n}$ and the fact that $v^{*} q_{n} v \in v^{*}(A q) v=B$ we derive that

$$
\begin{aligned}
\sum_{\gamma \in F_{n}^{\lambda}} \tau\left(\left|a_{\gamma}^{\lambda, n}\right|\right) & =\sum_{\gamma \in \Gamma} \tau\left(\left|a_{\gamma}^{\lambda, n}\right|\right)=\left\|q_{n} v v_{\lambda} v^{*} q_{n}\right\|_{2}^{2}=\tau\left(q_{n} v v_{\lambda} v^{*} q_{n} v v_{\lambda}^{*} v^{*} q_{n}\right) \\
& =\tau\left(v_{\lambda}\left(v^{*} q_{n} v\right) v_{\lambda}^{*}\left(v^{*} q_{n} v\right)\right)=\tau\left(\beta_{\lambda}\left(v^{*} q_{n} v\right)\left(v^{*} q_{n} v\right)\right) .
\end{aligned}
$$

Combining (7.g) and (7.i) yields

$$
\sum_{\gamma \in F_{n}^{\lambda}} \tau\left(\sigma_{\gamma}\left(q_{n}\right) q_{n}\right)=\tau\left(\beta_{\lambda}\left(v^{*} q_{n} v\right)\left(v^{*} q_{n} v\right)\right), \forall \lambda \in \Lambda .
$$

Recall that $\Lambda \cong \Gamma^{\prime} / K$ and let $\pi: \Gamma^{\prime} \rightarrow \Lambda$ be the projection given by $\pi(\delta(\lambda) k)=\lambda$, for all $\lambda \in \Lambda$ and $k \in K$. Let $\rho: \Gamma^{\prime} \rightarrow \operatorname{Aut}(A \bar{\otimes} B)$ be the action defined by $\rho_{g}=\sigma_{g} \otimes \beta_{\pi(g)}$, for all $g \in \Gamma^{\prime}$. Since $\sigma_{\mid \Gamma^{\prime}}$ and $\beta$ are both weakly mixing, we get that $\rho$ is weakly mixing. Let $\tau_{B}$ denote the normalized trace on $B$, i.e. $\tau_{B}(p)=1$ and $\tau_{B}(b)=\tau(p)^{-1} \tau(b)$, for all $b \in B$. Since $\rho$ is weakly mixing, we can find a sequence $\left\{g_{m}\right\}_{\geq 1} \subset \Gamma^{\prime}$ such that $\sigma_{g_{m}}(a) \rightarrow \tau(a)$ and $\beta_{\pi\left(g_{m}\right)}(b) \rightarrow \tau_{B}(b)$, weakly, for all $a \in A$ and $b \in B$, as $m \rightarrow \infty$. We may clearly assume that $g_{m}=\delta\left(\lambda_{m}\right) k$, for some sequence $\left\{\lambda_{m}\right\}_{m \geq 1} \subset \Lambda$ and $k \in K$. Thus, we have that $\sigma_{\delta\left(\lambda_{m}\right)}(a) \rightarrow \tau(a)$ and $\beta_{\lambda_{m}}(b) \rightarrow \tau_{B}(b)$, weakly, for all $a \in A, b \in B$.

In particular, it follows that we can find $\lambda \in \Lambda$ such that

$$
\begin{gathered}
\tau\left(\sigma_{\delta(\lambda) g}\left(q_{n}\right) q_{n}\right) \leq(1+\varepsilon) \tau\left(q_{n}\right)^{2}, \forall g \in F \text { and } \\
\tau\left(\beta_{\lambda}\left(v^{*} q_{n} v\right)\left(v^{*} q_{n} v\right)\right)=\tau(p) \tau_{B}\left(\beta_{\lambda}\left(v^{*} q_{n} v\right)\left(v^{*} q_{n} v\right)\right) \\
\geq(1-\varepsilon) \tau(p) \tau_{B}\left(v^{*} q_{n} v\right)^{2}=(1-\varepsilon) \tau(p)^{-1} \tau\left(q_{n}\right)^{2} .
\end{gathered}
$$

Since $F_{n}^{\lambda} \subset \delta(\lambda) F,(7 . j)$ and (7.k) together imply that $(1+\varepsilon)\left|F_{n}^{\lambda}\right|\left|\tau\left(q_{n}\right)\right|^{2} \geq$ $(1-\varepsilon) \tau(p)^{-1} \tau\left(q_{n}\right)^{2}$. Hence, $\left|F_{n}^{\lambda}\right| \geq(1-\varepsilon)^{2} \tau(p)^{-1}$.

From this, (7.g) and (7.h) we deduce that the cardinality of the set $S_{n}:=\{\gamma \in$ $\left.\Gamma|| c_{\gamma} \mid \geq\left(1-2^{-n}\right) \tau(p)\right\}$ is at least $(1-\varepsilon)^{2} \tau(p)^{-1}$. As $\varepsilon>0$ is arbitrary, we get that $\left|S_{n}\right| \geq \tau(p)^{-1}$, for all $n \geq 1$. Thus $S=\bigcap_{n \geq 1} S_{n}=\left\{\gamma|\in \Gamma|\left|c_{\gamma}\right| \geq \tau(p)\right\}$ has cardinality at least $\tau(p)^{-1}$. On the other hand, since $\sum_{\gamma \in \Gamma}\left|c_{\gamma}\right|^{2}=\|p\|_{2}^{2}=\tau(p)$, we get that $|S| \leq \tau(p)^{-1}$. The last two facts clearly give the claim. 
Going back to the proof of (2), notice that by combining the above claim and 34, Lemma 5.3 we can find a finite group $K^{\prime} \subset \Gamma$ and a character $\chi$ of $K^{\prime}$ such that $p=\left|K^{\prime}\right|^{-1} \sum_{k^{\prime} \in K^{\prime}} \chi\left(k^{\prime}\right) u_{k^{\prime}}$. Thus $K=\left\{\gamma \in \Gamma \mid u_{\gamma} p \in \mathbb{C} p\right\}=K^{\prime}$ and the last part of the proof of [34, Theorem 5.2 (pages 439-441) gives the conclusion.

\section{Strong RIGIDITY FOR EMBEDDINGS OF $\mathrm{II}_{1}$ FACTORS}

We are now ready to prove the main technical results of this paper. Let $M=$ $A \rtimes_{\sigma} \Gamma$ and $N=D \rtimes_{\rho} \Lambda$ be the crossed product algebras associated with actions of countable groups $\Gamma$ and $\Lambda$ on abelian von Neumann algebras $A$ and $D$. We say that an embedding $\Delta: N \rightarrow M$ is standard if we can find a subgroup $\Gamma_{0} \subset \Gamma$ and a $\sigma\left(\Gamma_{0}\right)$-invariant subalgebra $A_{0} \subset A$ such that, up to conjugation with a unitary element and modulo scalars, we have that $\Delta(\Lambda)=\Gamma_{0}$ and $\Delta(D)=A_{0}$. In other words, a standard embedding arises from a "realization" of $\Lambda$ as a subgroup $\Gamma_{0} \subset \Gamma$ and of $\rho$ as a quotient of $\sigma_{\mid \Gamma_{0}}$.

Assume that $\Lambda$ is a "rigid group" (e.g. $\Lambda$ has property (T) or is a product of two non-amenable groups) and that $\sigma$ is a Bernoulli action. Our first result roughly says that any embedding $\Delta: N \rightarrow M$ is standard unless it comes from an embedding of $N$ into $L(\Gamma)$. As we will see in Section 10, in certain situations (e.g., if $\Gamma=\mathbb{F}_{2} \times \mathbb{F}_{2}$ ), we can rule out the appearance of such bad embeddings and thus we can completely describe the embeddings of $N$ into $M$. This is why we use the phrase strong rigidity for embeddings to refer to the results of this section.

Theorem 8.1. Let $\Gamma$ be a torsion free, $I C C$, countable group, $B$ be an abelian von Neumann algebra and denote $M=B^{\Gamma} \rtimes \Gamma$. Let $\rho: \Lambda \rightarrow \operatorname{Aut}(D)$ be a free action of a countable group $\Lambda$ on an abelian von Neumann algebra $D$ and denote $N=D \rtimes_{\rho} \Lambda$. Suppose that $\Lambda$ admits an infinite, almost normal subgroup $\Lambda_{0}$ such that either

(1) the inclusion $\left(\Lambda_{0} \subset \Lambda\right)$ has the relative property $(T)$ or

(2) $\Lambda_{0}$ is generated by two commuting subgroups $\Lambda_{1}, \Lambda_{2}$, with $\Lambda_{1}$ non-amenable and $\Lambda_{2}$ infinite.

Let $\Delta: N \rightarrow M$ be a*-homomorphism and suppose that $\Delta(N) \nprec_{M} L(\Gamma)$.

Then $\Delta$ must be unital and there exist a character $\eta$ of $\Lambda$, a group homomorphism $\delta: \Lambda \rightarrow \Gamma$ and a unitary $u \in M$ such that $\Delta(D) \subset u A u^{*}$ and $\Delta\left(v_{\lambda}\right)=\eta(\lambda) u u_{\delta(\lambda)} u^{*}$, for all $\lambda \in \Lambda$.

Recall that a subgroup $\Lambda_{0}$ of a countable group $\Lambda$ is almost normal if $\lambda \Lambda_{0} \lambda^{-1} \cap \Lambda_{0}$ has finite index in both $\lambda \Lambda_{0} \lambda^{-1}$ and $\Lambda_{0}$, for any $\lambda \in \Lambda$.

We are also able to prove a strong rigidity result for the embeddings of $N$ into a tensor product $M=M_{1} \bar{\otimes} M_{2}$ of two $\mathrm{II}_{1}$ factors coming from Bernoulli actions. If we represent $M$ as a crossed product $A \rtimes \Gamma$, as in 3.1, then our second result gives a list of assumptions which force an embedding $\Delta: N \rightarrow M$ to be standard, in the sense from above.

This result will be crucial in deriving $W^{*}$-superrigidity (see Section 9 ).

Theorem 8.2. In the context from 3.1, i.e., $M_{i}=B_{i}^{\Gamma_{i}} \rtimes \Gamma_{i}, M=M_{1} \bar{\otimes} M_{2}$, $A=B_{1}^{\Gamma_{1}} \bar{\otimes} B_{2}^{\Gamma_{2}}, \Gamma=\Gamma_{1} \times \Gamma_{2}$, assume that $\Gamma$ is $I C C$ (i.e. $\Gamma_{1}, \Gamma_{2}$ are ICC).

Let $\rho: \Lambda \rightarrow \operatorname{Aut}(D)$ be a free action of a countable group $\Lambda$ on an abelian von Neumann algebra $D$ and denote $N=D \rtimes_{\rho} \Lambda$. Suppose that $\Lambda$ admits an infinite, almost normal subgroup $\Lambda_{0}$ such that the inclusion $\left(\Lambda_{0} \subset \Lambda\right)$ has the relative property $(T)$. 
Let $\Delta: N \rightarrow M$ be a $*$-homomorphism and suppose that:

(1) $\Delta\left(L\left(\Lambda_{0}\right)\right) \nprec_{M} L\left(\Gamma_{1}\right) \otimes 1$ and $\Delta\left(L\left(\Lambda_{0}\right)\right) \nprec_{M} 1 \otimes L\left(\Gamma_{2}\right)$.

(2) $\Delta(N) \nprec_{M} L\left(\Gamma_{1}\right) \bar{\otimes} M_{2}$ and $\Delta(N) \nprec_{M} M_{1} \bar{\otimes} L\left(\Gamma_{2}\right)$.

We have that

- If $\Gamma$ is torsion free, then $\Delta$ must be unital and we can find a character $\eta$ of $\Lambda, a$ group homomorphism $\delta: \Lambda \rightarrow \Gamma$ and a unitary $u \in M$ such that $\Delta(D) \subset u A u^{*}$ and $\Delta\left(v_{\lambda}\right)=\eta(\lambda) u u_{\delta(\lambda)} u^{*}$, for all $\lambda \in \Lambda$.

- In general, we can find a finite index subgroup $\Lambda_{1}$ of $\Lambda$, a 1-1 map $\delta: \Lambda_{1} \rightarrow \Gamma$, $u_{1}, \ldots, u_{n} \in \mathcal{N}_{M}(A)$ and $u \in \mathcal{U}(M)$ such that if we let $\tilde{\Delta}=\operatorname{Ad}(u) \circ \Delta: N \rightarrow M$, then

(a) $\tilde{\Delta}(D) \subset A$, there exists a projection $0 \neq p \in A$ and $a_{i}^{\lambda} \in(A)_{1}$, for all $i \in$ $\{1, \ldots, n\}, \lambda \in \Lambda_{1}$, such that $p \leq \tilde{\Delta}(1)$ and $p \tilde{\Delta}\left(v_{\lambda}\right)=\sum_{i=1}^{n} a_{i}^{\lambda} u_{\delta(\lambda)} u_{i}$, for all $\lambda \in \Lambda_{1}$,

(b) there exists a finite subgroup $K \subset \Gamma$ such that $\delta(\lambda)$ normalizes $K$ and we have that $\delta\left(\lambda \lambda^{\prime}\right)^{-1} \delta(\lambda) \delta\left(\lambda^{\prime}\right)$ belongs to $K$, for all $\lambda, \lambda^{\prime} \in \Lambda_{1}$.

If in addition $\max \{|K| \mid K$ finite subgroup of $\Gamma\}<\infty$, then $\tau(\Delta(1)) \in l^{-1} \mathbb{N}$, where $l$ is the least common multiple of $|K|$ with $K$ being a finite subgroup of $\Gamma$.

The conclusion of Theorem 8.2 (in the case when $\Gamma$ has torsion) is rather involved and technical and might seem hard to work with.

However, it is manageable enough to be useful in applications. Thus, we will use it to prove $W^{*}$-superrigidity of Bernoulli actions of property (T) groups without assuming torsion freeness. This is of particular interest when applied to the linear groups $\mathrm{SL}_{n}(\mathbb{Z})$, for $n \geq 3$.

Theorem 8.1 can also be extended (just as Theorem 8.2) to cover groups with torsion. On the other hand, note that Theorem 8.1 applies to a larger class of groups $\Lambda$ than Theorem 8.2. Let us explain why this difference appears. The first step in the proofs of both Theorems 8.1 and 8.2 consists of showing that if $\Delta: N \rightarrow M$ is an embedding, then $L(\Lambda)$ is "absorbed" by $L(\Gamma)$. When $\Lambda$ has property $(\mathrm{T})$ this is granted by Theorem 2.1, in both the contexts from Theorems 8.1 and 8.2. If $\Lambda=\Lambda_{1} \times \Lambda_{2}$ is a product of two non-amenable groups, the absorption result still holds in the context of Theorem 8.1 (by [39). However, if $M=M_{1} \bar{\otimes} M_{2}$ is as in Theorem 8.2, then the absorption result fails in general. Indeed, one can easily construct a situation in which we have that $L\left(\Lambda_{1}\right) \subset M_{1}$ and $L\left(\Lambda_{2}\right) \subset M_{2}$ without having that $L(\Lambda)$ is absorbed by $L(\Gamma)$.

We first prove Theorem 8.2. Since the proof of Theorem 8.1 is analogous to the proof of Theorem 8.2, we will only sketch it, leaving most of the details to the reader.

Proof of Theorem 8.2. Let $\Delta: N \rightarrow M$ be a $*$-homomorphism satisfying (1)-(2) and set $q=\Delta(1)$.

From now on, we identify $N$ with $\Delta(N)$ and forget about $\Delta$. Since the inclusion $\left(\Lambda_{0} \subset \Lambda\right)$ has the relative property $(\mathrm{T})$, the inclusion $\left(L\left(\Lambda_{0}\right) \subset q M q\right)$ is rigid (35]). By using (1) and the fact that $\Gamma$ is ICC, Theorem 2.1 implies that we can find a unitary $u \in M$ such that $u\left(q \mathcal{N}_{q M q}\left(L\left(\Lambda_{0}\right)\right)^{\prime \prime}\right) u^{*} \subset L(\Gamma)$. Since $\Lambda_{0}$ is almost normal in $\Lambda$ we deduce that

$$
u L(\Lambda) u^{*} \subset L(\Gamma)
$$


Next, we claim that

$$
D \nprec_{M} L\left(\Gamma_{1}\right) \bar{\otimes} M_{2} \text { and } D \nprec_{M} M_{1} \bar{\otimes} L\left(\Gamma_{2}\right) .
$$

Assume by contradiction that $D \prec_{M} L\left(\Gamma_{1}\right) \bar{\otimes} M_{2}$. Since $D$ is abelian, we can find a non-zero projection $r \in D^{\prime} \cap q M q$ and $a, b \in M$ such that $(D)_{1} r \subset$ $a\left(L\left(\Gamma_{1}\right) \bar{\otimes} M_{2}\right)_{1} b$. Recall that $M_{1}=B_{1}^{\Gamma_{1}} \rtimes \Gamma_{1}$ and let $\tilde{M}_{1} \supset M_{1},\left\{\theta_{t}^{1}\right\}_{t \in \mathbb{R}} \subset \operatorname{Aut}\left(\tilde{M}_{1}\right)$ be the weakly malleable deformations defined in section 1.5. Set $\theta_{t}=\theta_{t}^{1} \otimes \operatorname{id}_{M_{2}} \in$ $\operatorname{Aut}\left(\tilde{M}_{1} \bar{\otimes} M_{2}\right)$.

Let $\mathcal{V}$ be a $\rho(\Lambda)$-invariant, $\|.\|_{2}$-dense subgroup of $\mathcal{U}(D)$. Then $\mathcal{U}=\left\{v_{\lambda} v \mid \lambda \in\right.$ $\Lambda, v \in \mathcal{V}\}$ is a group of unitaries of $N$ which generates $N$ as a von Neumann algebra. By using (8.a) and the inclusion $\mathcal{V} r \subset a\left(M_{2}\right)_{1} b$, we get that $\mathcal{U} r \subset$ $u^{*}(L(\Gamma))_{1} u a\left(L\left(\Gamma_{1}\right) \bar{\otimes} M_{2}\right)_{1} b$. Since $\theta_{t \mid L\left(\Gamma_{1}\right) \bar{\otimes} M_{2}}=\mathrm{id}_{L\left(\Gamma_{1}\right) \bar{\otimes} M_{2}}$, we can find $t>0$ such that $\left\|\theta_{t}(x) r-x r\right\|_{2} \leq \frac{\|r\|_{2}}{2}$, for all $x \in \mathcal{U}$. Thus, for all $x \in \mathcal{U}$ we have that $\Re\left\langle\left(\theta_{t}(x) r-x r\right), x r\right\rangle \geq-\frac{\|r\|_{2}^{2}}{2}$; hence

$$
\Re \tau\left(x^{*} \theta_{t}(x) r\right)=\Re\left\langle\theta_{t}(x) r, x r\right\rangle=\langle x r, x r\rangle+\Re\left\langle\left(\theta_{t}(x) r-x r\right), x r\right\rangle \geq \frac{\|r\|_{2}^{2}}{2} .
$$

Since $\mathcal{U}$ is a group, by averaging (see e.g. the proof of Theorem 2.1) we deduce that there exists $0 \neq v \in q M$ such that $v \theta_{t}(x)=x v$, for all $x \in \mathcal{U}$. Since $\mathcal{U}^{\prime \prime}=N$, we get that $v \theta_{t}(x)=x v$, for all $x \in N$.

The proof of Theorem 2.1 (see Remark 2.2) implies that $N \prec_{M} L\left(\Gamma_{1}\right) \bar{\otimes} M_{2}$. This contradicts assumption (2); hence (8.b) is proven.

Further, by combining (8.a), (1) and (8.b), Theorem 6.1 implies that $C=D^{\prime} \cap$ $q M q$ is a type I von Neumann algebra and that we may assume that $q=\Delta(1) \in A$ and $A q \subset C$.

Denote by $\mathcal{Z}$ the center of $C$. Since $D$ is abelian, $\mathcal{Z}$ contains $D$. Since $C$ is of type I we can decompose $\mathcal{Z}=\bigoplus_{i>1} \mathcal{Z}_{i}$, where the $\mathcal{Z}_{i}$ are abelian von Neumann algebras, such that $C=\bigoplus_{i>1}\left(\mathbb{M}_{n_{i}}(\mathbb{C}) \otimes \mathcal{Z}_{i}\right)$, for some (possibly finite) strictly increasing sequence $1 \leq n_{1}<n_{2}<\cdots$. Since $A \subset M$ is maximal abelian, we get that $A q$ is maximal abelian in $C$. Since, by a classical result, any two maximal abelian subalgebras of a type I algebra are conjugate (see e.g. [44, Lemma C.2), we may assume that $A q=\bigoplus_{i>1}\left(\mathbb{C}^{n_{i}} \otimes \mathcal{Z}_{i}\right)$, where $\mathbb{C}^{n_{i}} \subset \mathbb{M}_{n_{i}}(\mathbb{C})$ is the subalgebra of diagonal matrices.

Now, since $\Lambda=\left\{v_{\lambda}\right\}_{\lambda \in \Lambda} \subset \mathcal{U}(q M q)$ normalizes $D$, it also normalizes $C=$ $D^{\prime} \cap q M q$ and $\mathcal{Z}=\mathcal{Z}(C)$. Moreover, $\Lambda$ normalizes $\mathbb{M}_{n_{i}}(\mathbb{C}) \otimes \mathcal{Z}_{i}$ and $\mathcal{Z}_{i}$, for all $i \geq 1$. Denote by $\alpha$ the action of $\Lambda$ on $\mathcal{Z}$ given by $\alpha_{\lambda}(z)=v_{\lambda} z v_{\lambda}^{*}$, for all $\lambda \in \Lambda$ and $z \in \mathcal{Z}$. Since $\alpha$ leaves $\mathcal{Z}_{i}$ invariant, for all $i \geq 1$, we can define an action $\beta$ of $\Lambda$ on $C$ by letting

$$
\beta_{\lambda}=\bigoplus_{i \geq 1}\left(\operatorname{id}_{\mathbb{M}_{n_{i}}(\mathbb{C})} \otimes \alpha_{\lambda \mid \mathcal{Z}_{i}}\right) \in \operatorname{Aut}(C), \forall \lambda \in \Lambda
$$

Let $\lambda \in \Lambda$. Since the automorphisms $\beta_{\lambda}$ and $\operatorname{Ad}\left(v_{\lambda}\right)$ of $C$ are equal on its center, $\mathcal{Z}$, by [19], Corollary 9.3.5 we can find a unitary $\omega_{\lambda} \in C$ such that $\beta_{\lambda}=\operatorname{Ad}\left(v_{\lambda} \omega_{\lambda}\right)$, as automorphisms of $C$. As $\beta$ leaves $A q=\bigoplus_{i \geq 1}\left(\mathbb{C}^{n_{i}} \otimes \mathcal{Z}_{i}\right)$ invariant, we get that $v_{\lambda} \omega_{\lambda} \in \mathcal{N}_{q M q}(A q)$. Thus, we can find $z_{\lambda} \in \mathcal{U}(\overline{A q})$ and $V_{\lambda} \in \mathcal{N}_{q M q}(A q)$ of the form $V_{\lambda}=\sum_{\gamma \in \Gamma} p_{\gamma, \lambda} u_{\gamma}$, where $p_{\gamma, \lambda} \in \mathcal{P}(A q)$, such that $v_{\lambda} \omega_{\lambda}=V_{\lambda} z_{\lambda}$.

We claim that $V_{\lambda} V_{\lambda^{\prime}}=V_{\lambda \lambda^{\prime}}$, for all $\lambda, \lambda^{\prime} \in \Lambda$. To see this, let $v=V_{\lambda \lambda^{\prime}}^{*} V_{\lambda} V_{\lambda^{\prime}} \in$ $\mathcal{U}(q M q)$. Note that for all $x \in A q$ we have that $V_{\lambda} x V_{\lambda}^{*}=V_{\lambda} z_{\lambda} x z_{\lambda}^{*} V_{\lambda}^{*}=\beta_{\lambda}(x)$. Using this fact, we immediately get that $v \in(A q)^{\prime} \cap q M q=A q$. On the other hand, 
$v$ is of the form $v=\sum_{\gamma \in \Gamma} p_{\gamma} u_{\gamma}$, for some $p_{\gamma} \in \mathcal{P}(A q)$ (this is because $V_{\lambda}, V_{\lambda^{\prime}}$ and $V_{\lambda \lambda^{\prime}}^{*}$ are of this form). By combining the last two observations we get that $v=1$, as claimed.

We also claim that $E_{A}\left(V_{\lambda}\right)=0$, for all $\lambda \in \Lambda \backslash\{e\}$. Otherwise, we can find $\lambda \neq e$ and a non-zero projection $p \in A q$ such that $\beta_{\lambda}(x)=x$, for all $x \in A p$. Thus, we can find a non-zero projection $p_{0} \in \mathcal{Z}$ such that $\alpha_{\lambda}(x)=x$, for all $x \in \mathcal{Z} p_{0}$. Since $D \subset \mathcal{Z}$, we get that $\alpha_{\lambda}(x) p_{0}=x p_{0}$, for all $x \in D$. Let $0 \neq p_{1} \in D$ be the support projection of $E_{D}\left(p_{0}\right)$. By projecting onto $D$ and noticing that $\alpha_{\lambda \mid D}=\rho_{\lambda}$, as automorphisms of $D$, we get that $\rho_{\lambda}(x) p_{1}=x p_{1}$, for all $x \in D$. This, however, contradicts the freeness of $\rho$.

Altogether, we proved that there exist $w_{\lambda}=\omega_{\lambda} z_{\lambda}^{*} \in \mathcal{U}(C)$ such that $V_{\lambda}=v_{\lambda} w_{\lambda}$ normalizes $C, A q$ and $\mathcal{Z}, V_{\lambda} V_{\lambda^{\prime}}=V_{\lambda \lambda^{\prime}}$ and $E_{A}\left(V_{\lambda}\right)=\delta_{\lambda, e} q$, for all $\lambda, \lambda^{\prime} \in \Lambda$. For $i \geq 1$, let $e_{i, 1}, \ldots, e_{i, n_{i}} \in \mathbb{C}^{n_{i}}$ be the canonical projections. Then $\beta$ fixes $p_{i, j}:=e_{i, j} \otimes 1 \in \mathbb{C}^{n_{i}} \otimes \mathcal{Z}_{i}$.

Next, we prove the following:

Claim 1. There exists a unitary $v \in \mathcal{U}(M)$ such that $v V_{\lambda} v^{*} \in L(\Gamma)$, for all $\lambda \in \Lambda$.

Proof of Claim 1. Let $Q=\left\{V_{\lambda} \mid \lambda \in \Lambda_{0}\right\}^{\prime \prime}$ and $P=\left\{V_{\lambda} \mid \lambda \in \Lambda\right\}^{\prime \prime}$. Since the inclusion $\left(\Lambda_{0} \subset \Lambda\right)$ has the relative property $(\mathrm{T})$, by [35] the inclusion $(Q \subset M)$ is rigid. Since $\Lambda_{0}$ is almost normal in $\Lambda$, we get that $P \subset q \mathcal{N}_{q M q}(Q)^{\prime \prime}$. Since $\Gamma$ is ICC, by Theorem 2.1, in order to prove the claim, it suffices to show that $Q \nprec_{M} L\left(\Gamma_{1}\right)$ and $Q \nprec_{M} L\left(\Gamma_{2}\right)$.

Let us show that $Q \nprec_{M} L\left(\Gamma_{1}\right)$. Since $L\left(\Lambda_{0}\right) \nprec_{M} L\left(\Gamma_{1}\right)$, Theorem 1.3.1 gives a sequence $\left\{\lambda_{n}\right\}_{n \geq 1} \subset \Lambda_{0}$ satisfying $\left\|E_{L\left(\Gamma_{1}\right)}\left(x v_{\lambda_{n}} y\right)\right\|_{2} \rightarrow 0$, for all $x, y \in M$. We claim that

$$
\sup _{a \in(A)_{1}}\left\|E_{L\left(\Gamma_{1}\right)}\left(x v_{\lambda_{n}} y a z\right)\right\|_{2} \rightarrow 0, \forall x, y, z \in M .
$$

Let $a \in(A)_{1}$. Since $u L\left(\Lambda_{0}\right) u^{*} \subset L(\Gamma)$, we can assume that $v_{\lambda_{n}} \in L(\Gamma)$, for the purpose of proving (8.c). Then we can decompose $v_{\lambda_{n}}=\sum_{\gamma \in \Gamma} c_{n, \gamma} u_{\gamma}$, where $c_{n, \gamma} \in \mathbb{C}$, for all $n \geq 1$ and $\gamma \in \Gamma$. It is clearly enough to prove (8.c) when $x=x_{0} u_{g}, y=y_{0} u_{h}, z=z_{0} u_{k}$, for some $x_{0}, y_{0}, z_{0} \in(A)_{1}$ and $g, h, k \in \Gamma$. In this case, we have that

$$
x v_{\lambda_{n}} y a z=\sum_{\gamma \in \Gamma} c_{n, \gamma} x_{0} \sigma_{g \gamma}\left(y_{0}\right) \sigma_{g \gamma h}\left(a z_{0}\right) u_{g \gamma h k} .
$$

Since $a, x_{0}, y_{0}, z_{0} \in(A)_{1}$, we get that $\left|\tau\left(x_{0} \sigma_{g \gamma}\left(y_{0}\right) \sigma_{g \gamma h}\left(a z_{0}\right)\right)\right| \leq 1$, for all $\gamma \in \Gamma$. Thus

$$
\begin{aligned}
\left\|E_{L\left(\Gamma_{1}\right)}\left(x v_{\lambda_{n}} y a z\right)\right\|_{2}^{2} & =\sum_{\gamma \in g^{-1} \Gamma_{1} k^{-1} h^{-1}}\left|c_{n, \gamma}\right|^{2}\left|\tau\left(x_{0} \sigma_{g \gamma}\left(y_{0}\right) \sigma_{g \gamma h}\left(a z_{0}\right)\right)\right|^{2} \\
& \leq \sum_{\gamma \in g^{-1} \Gamma_{1} k^{-1} h^{-1}}\left|c_{n, \gamma}\right|^{2}=\left\|E_{L\left(\Gamma_{1}\right)}\left(u_{g} v_{\lambda_{n}} u_{h k}\right)\right\|_{2}^{2} .
\end{aligned}
$$

As we have that $\left\|E_{L\left(\Gamma_{1}\right)}\left(u_{g} v_{\lambda_{n}} u_{h k}\right)\right\|_{2} \rightarrow 0$, (8.c) is therefore proven.

Next, fix $\varepsilon>0$ and $x, y \in M$. Let $q_{0} \in C$ be a projection such that $\left\|q_{0}-q\right\|_{2} \leq$ $\varepsilon$ and $C q_{0}=\bigoplus_{i=1}^{k}\left(\mathbb{M}_{n_{i}}(\mathbb{C}) \otimes \mathcal{Z}_{i}\right)$, for some $k \geq 1$. Remark that we can find $z_{1}, \ldots, z_{l} \in C$ such that $\left(C q_{0}\right)_{1} \subset \sum_{j=1}^{l}(A)_{1} z_{j}$. Moreover, for every $u \in \mathcal{U}(C)$ we have that $\left\|u\left(q-q_{0}\right)\right\|_{2}=\left\|q-q_{0}\right\|_{2} \leq \varepsilon$. By using these facts and recalling that 
$w_{\lambda_{n}} \in \mathcal{U}(C)$, we deduce that

$$
\begin{aligned}
& \left\|E_{L\left(\Gamma_{1}\right)}\left(x V_{\lambda_{n}} y\right)\right\|_{2}=\left\|E_{L\left(\Gamma_{1}\right)}\left(x v_{\lambda_{n}} w_{\lambda_{n}} y\right)\right\|_{2} \\
& \quad \leq\left\|E_{L\left(\Gamma_{1}\right)}\left(x v_{\lambda_{n}} w_{\lambda_{n}}\left(q-q_{0}\right) y\right)\right\|_{2}+\left\|E_{L\left(\Gamma_{1}\right)}\left(x v_{\lambda_{n}}\left(w_{\lambda_{n}} q_{0}\right) y\right)\right\|_{2} \\
& \quad \leq \varepsilon\|x\|\|y\|+\sum_{j=1}^{l} \sup _{a \in(A)_{1}}\left\|E_{L\left(\Gamma_{1}\right)}\left(x v_{\lambda_{n}} a z_{j} y\right)\right\|_{2} \quad \forall n \geq 1 .
\end{aligned}
$$

This inequality in combination with (8.c) gives that $\lim \sup _{n \rightarrow \infty}\left\|E_{L\left(\Gamma_{1}\right)}\left(x V_{\lambda_{n}} y\right)\right\|_{2}$ $\leq \varepsilon$. Since $\varepsilon>0$ is arbitrary, we deduce that $\lim _{n \rightarrow \infty}\left\|E_{L\left(\Gamma_{1}\right)}\left(x V_{\lambda_{n}} y\right)\right\|_{2}=0$, for all $x, y \in M$. Since $\left\{V_{\lambda_{n}}\right\}_{n \geq 1} \subset \mathcal{U}(Q)$, we get that $Q \nprec_{M} L\left(\Gamma_{1}\right)$.

Next, recall that $V_{\lambda}$ normalizes $A q$ and $V_{\lambda} x V_{\lambda}^{*}=\beta_{\lambda}(x)$, for all $x \in A q, \lambda \in \Lambda$.

Claim 2 ([34]). There exist a finite index subgroup $\Lambda_{1} \subset \Lambda$ and a non-zero $\beta\left(\Lambda_{1}\right)$ invariant projection $p \in A q \cap v^{*} L(\Gamma) v$ such that the restriction of the action $\beta_{\mid \Lambda_{1}}$ to $A p$ is weakly mixing. Moreover, we have that $p \leq p_{i, j}$, for some $i \geq 1$ and $j \in\left\{1, \ldots, n_{i}\right\}$.

Proof of Claim 2. This claim follows from an argument of Popa (see the proofs of [34, Lemma 4.5 and [44, Theorem 9.1). Let $A_{0}=A q \cap v^{*} L(\Gamma) v$ and note that $q \in A_{0}$. It is easy to see that $A_{0}$ is a completely atomic, (abelian) von Neumann algebra. Since $V_{\lambda} \in v^{*} L(\Gamma) v$, for all $\lambda \in \Lambda$, we get that $V_{\lambda}$ normalizes $A_{0}$; hence $A_{0}$ is $\beta$-(globally) invariant. Let $p \in A_{0}$ be a minimal projection. Then $p$ is invariant under some finite index subgroup $\Lambda_{1}$ of $\Lambda$. Denote $Q_{1}:=\left\{V_{\lambda} \mid \lambda \in \Lambda_{1}\right\}^{\prime \prime} \subset q M q$ and notice that $p \in Q_{1}^{\prime} \cap q M q$.

Towards proving that the restriction of $\beta_{\mid \Lambda_{1}}$ to $A p$ is weakly mixing, let $\mathcal{H} \subset A p$ be a finite dimensional $\beta\left(\Lambda_{1}\right)$-invariant subspace. Since for every $\lambda \in \Lambda_{1}$ and $\xi \in \mathcal{H}$, we have that $V_{\lambda} \xi=\beta_{\lambda}(\xi) V_{\lambda} \subset \mathcal{H} V_{\lambda}$ and $\xi V_{\lambda}=V_{\lambda} \beta_{\lambda^{-1}}(\xi) \subset V_{\lambda} \mathcal{H}$, we derive that $\mathcal{H}$ is contained in the quasi-normalizer of $Q_{1} p$ in $p M p$.

Since $Q_{1} p \subset v^{*} L(\Gamma) v$, while $Q_{1} \nprec_{M} L\left(\Gamma_{1}\right)$ and $Q_{1} \nprec_{M} L\left(\Gamma_{2}\right)$ (by the proof of Claim 1), [45], Lemma 4.2 gives that $\mathcal{H} \subset v^{*} L(\Gamma) v$. Thus, we get that $\mathcal{H} \subset$ $A p \cap v^{*} L(\Gamma) v=A_{0} p$. Since $p$ is a minimal projection in $A_{0}$, we must have that $\mathcal{H}=\mathbb{C} p$, which proves the weak mixingness assertion.

The moreover assertion is clear since $p_{i, j}$ is $\beta$-invariant, for all $i \geq 1, j \in$ $\left\{1, \ldots, n_{i}\right\}$.

To summarize, we have that

- $\left\{V_{\lambda}\right\}_{\lambda \in \Lambda} \subset v^{*} L(\Gamma) v \cap \mathcal{U}(q M q)$ normalizes $A q$,

- $p \in A q \cap v^{*} L(\Gamma) v$ commutes with $V_{\lambda}$, for all $\lambda \in \Lambda_{1}$, and

- the action $\Lambda_{1} \ni \lambda \rightarrow \beta_{\lambda \mid A p}=\operatorname{Ad}\left(V_{\lambda} p\right) \in \operatorname{Aut}(A p)$ is weakly mixing. Let $p^{\prime}=v p v^{*} \in L(\Gamma)$ and $\Lambda_{1}=\left\{v\left(V_{\lambda} p\right) v^{*}\right\}_{\lambda \in \Lambda_{1}} \subset \mathcal{U}\left(p^{\prime} L(\Gamma) p^{\prime}\right)$. Then $\Lambda_{1}$ acts on $B=v(A p) v^{*} \subset p^{\prime}\left(A \rtimes_{\sigma} \Gamma\right) p^{\prime}$ by conjugation and the resulting action (denoted $\beta^{\prime}$ ) is weakly mixing. Moreover, we have that $\left\{B, \Lambda_{1}\right\}^{\prime \prime} \cong B \rtimes_{\beta^{\prime}} \Lambda_{1}$. Indeed, this is because $E_{B}\left(v\left(V_{\lambda} p\right) v^{*}\right)=E_{A p}\left(V_{\lambda} p\right)=E_{A}\left(V_{\lambda}\right) p=0$, for all $\lambda \in \Lambda_{1} \backslash\{e\}$.

By applying Theorem 7.1 we can find maps $h: \Lambda_{1} \rightarrow S^{1}, \delta: \Lambda_{1} \rightarrow \Gamma$ and a unitary $w \in L(\Gamma)$ such that $\left[w u_{\delta(\lambda)} w^{*}, p^{\prime}\right]=0$ and

$$
v\left(V_{\lambda} p\right) v^{*}=h(\lambda)\left(w u_{\delta(\lambda)} w^{*}\right) p^{\prime}, \forall \lambda \in \Lambda_{1} .
$$

Let $K=\left\{\gamma \in \Gamma \mid\left(w u_{\gamma} w^{*}\right) p^{\prime} \in \mathbb{C} p^{\prime}\right\}$ and $\Gamma^{\prime}=\left\{\delta(\lambda) k \mid \lambda \in \Lambda_{1}, k \in K\right\}$. Then $K, \Gamma^{\prime}$ are subgroups of $\Gamma,|K|<\infty$ and $\Gamma^{\prime}$ normalizes $K$. It is also clear that 
$\delta\left(\lambda \lambda^{\prime}\right)^{-1} \delta(\lambda) \delta\left(\lambda^{\prime}\right) \in K$, for all $\lambda, \lambda^{\prime} \in \Lambda_{1}$, thus proving condition (b) in the general case.

To show that $\sigma_{\mid \Gamma^{\prime}}$ is weakly mixing, let $\pi_{i}: \Gamma \rightarrow \Gamma_{i}$ be the projection $\pi_{i}\left(\gamma_{1}, \gamma_{2}\right)=$ $\gamma_{i}$. Then $\sigma$ is the diagonal product of the generalized Bernoulli actions $\sigma_{i} \circ \pi_{i}: \Gamma \rightarrow$ $\operatorname{Aut}\left(B_{i}^{\Gamma_{i}}\right)$. Thus, if $\sigma_{\mid \Gamma^{\prime}}$ is not weakly mixing, then either $\left(\sigma_{1} \circ \pi_{1}\right)_{\mid \Gamma^{\prime}}$ or $\left(\sigma_{2} \circ \pi_{2}\right)_{\mid \Gamma^{\prime}}$ is not weakly mixing. By [37, Lemma 4.5 we would get that some finite index subgroup $\Gamma^{\prime \prime}$ of $\Gamma^{\prime}$ is contained in either $\Gamma_{1}$ or $\Gamma_{2}$. By using (8.d), this would imply that a corner of $Q_{1}=\left\{V_{\lambda} \mid \lambda \in \Lambda_{1}\right\}^{\prime \prime}$ embeds into either $L\left(\Gamma_{1}\right)$ or $L\left(\Gamma_{2}\right)$, a contradiction, by the proof of Claim 1 .

For the rest of the proof we analyze separately the case when $\Gamma$ is torsion free and the general case:

- If $\Gamma$ is torsion free, then $K=\{e\}, h$ is a character, $\delta$ is a $1-1$ homomorphism and $\Gamma^{\prime}=\delta\left(\Lambda_{1}\right)$. Since $\sigma_{\mid \delta\left(\Lambda_{1}\right)}$ is ergodic, by Theorem 7.1 we get that $p=1$ and $B=w A w^{*}$.

Since $p \leq q=\Delta(1), \Delta$ must be unital. Also, since by Claim 2 we have that $p \leq p_{i, j}$, for some $i \geq 1$ and $j \in\left\{1, \ldots, n_{i}\right\}$, we deduce that $C$ is abelian and $\mathcal{Z}=A=C$. Moreover, we get that $\beta_{\mid \Lambda_{1}}$ is weakly mixing and hence $\beta$ itself is weakly mixing. Thus, in Claim 2 and after we may take $\Lambda_{1}=\Lambda$. If $z=v^{*} w$, then $z$ normalizes $A$ and (8.d) can be rewritten as

$$
V_{\lambda}=h(\lambda) z u_{\delta(\lambda)} z^{*}, \forall \lambda \in \Lambda .
$$

Let $\theta=\operatorname{Ad}(z) \in \operatorname{Aut}(A)$. Then (8.e) implies that $\beta_{\lambda}=\theta \circ \sigma_{\delta(\lambda)} \circ \theta^{-1}$, for all $\lambda \in \Lambda$. In other words, the actions $\beta$ and $\sigma_{\mid \delta(\Lambda)}$ are conjugate.

Next, recall that $v_{\lambda}=V_{\lambda} w_{\lambda}^{*}$, for all $\lambda \in \Lambda$, for some unitary $w_{\lambda} \in C=A$. Since $v_{\lambda} v_{\lambda^{\prime}}=v_{\lambda \lambda^{\prime}}$ and $v_{\lambda}=\beta_{\lambda}\left(w_{\lambda}^{*}\right) V_{\lambda}$, for all $\lambda, \lambda^{\prime} \in \Lambda$, we get that the map $\Lambda \ni \lambda \rightarrow$ $\beta_{\lambda}\left(w_{\lambda}^{*}\right) \in \mathcal{U}(A)$ is a 1 -cocycle for $\beta$. By the proof of Claim $2, Q=\left\{V_{\lambda} \mid \lambda \in \Lambda_{0}\right\}^{\prime \prime}$ satisfies $Q \nprec_{M} L\left(\Gamma_{1}\right)$ and $Q \nprec_{M} L\left(\Gamma_{2}\right)$. By reasoning as above we deduce that $\sigma_{\mid \delta\left(\Lambda_{0}\right)}$ is weakly mixing. Note that the inclusion $\left(\delta\left(\Lambda_{0}\right) \subset \delta(\Lambda)\right)$ has the relative property $(\mathrm{T})$ and is almost normal. Finally, note that $\sigma$ is $s$-malleable (see [37, section 4 for the definition and proof).

Altogether, we can apply Popa's cocycle superrigidity theorem [37, Corollary 5.2 and deduce that the action $\sigma_{\mid \delta(\Lambda)}$ is $\mathcal{U}_{\text {fin }}$-cocycle superrigid (in the sense of [37, 5.6.0). Thus, $\beta_{\mid \Lambda}$ is cocycle superrigid, so we can find $U \in \mathcal{U}(A)$ and a character $h^{\prime}$ of $\Lambda$ such that $\beta(\lambda)\left(w_{\lambda}^{*}\right) V_{\lambda}=h^{\prime}(\lambda) U V_{\lambda} U^{*}$, for all $\lambda \in \Lambda$. Together with (8.e) this provides a character $\eta=h h^{\prime}$ of $\Lambda$ and $W=U z \in \mathcal{N}_{M}(A)$ such that $v_{\lambda}=\eta(\lambda) W u_{\delta(\lambda)} W^{*}$, for all $\lambda \in \Lambda$. As $A=C \supset D$, this gives the conclusion in the case when $\Gamma$ is torsion free.

- For general $\Gamma$, since $\sigma_{\mid \Gamma^{\prime}}$ is weakly mixing, by Theorem 7.1 we deduce that $p_{0}=$ $w^{*} p^{\prime} w$ is of the form $p_{0}=|K|^{-1} \sum_{k \in K} \chi(k) u_{k} \in L(K)$, for some character $\chi$ of $K$, and that $B=w\left(A^{K} p_{0}\right) w^{*}$, where $A^{K}=\{a \in A \mid \sigma(k)(a)=a, \forall k \in K\}$.

In particular, this shows that $\tau(p)=|K|^{-1} \in l^{-1} \mathbb{N}$, for every minimal projection $p$ of $A q \cap v L(\Gamma) v^{*}$ and so we get that $\tau(\Delta(1))=\tau(q) \in l^{-1} \mathbb{N}$ (if we assume that $l<\infty)$.

Now, let $z=v^{*} w$. Then (8.d) implies that $V_{\lambda} p=h(\lambda) z\left(u_{\delta(\lambda)} p_{0}\right) z^{*}$, for all $\lambda \in \Lambda_{1}$. Also, since $B=v(A p) v^{*}=w\left(A^{K} p_{0}\right) w^{*}$, we get that

$$
A p=z\left(A^{K} p_{0}\right) z^{*} \text {. }
$$

Next, let $q_{0} \in A$ be a projection such that the projections $\left\{\sigma(k)\left(q_{0}\right)\right\}_{k \in K}$ form a partition of unity in $A$ (here, we use the fact that $K$ acts freely). Then it is easy 
to verify that $\xi=|K|^{\frac{1}{2}} p_{0} q_{0}$ is a partial isometry such that $\xi^{*} \xi=q_{0}, \xi \xi^{*}=p_{0}$ and $A^{K} p_{0}=\xi\left(A q_{0}\right) \xi^{*}$. Thus, (8.f) gives that $z \xi\left(A q_{0}\right)(z \xi)^{*}=A p$ and so we can find $\eta \in \mathcal{N}_{M}(A)$ extending the partial isometry $z \xi$. Since $\eta \xi^{*}=z p_{0}$ we get that

$$
V_{\lambda} p=h(\lambda) \eta\left(\xi^{*} u_{\delta(\lambda)} \xi\right) \eta^{*}, \forall \lambda \in \Lambda_{1} .
$$

Recall that $v_{\lambda}=V_{\lambda} w_{\lambda}^{*}$, where $w_{\lambda} \in \mathcal{U}(C)$, and that $C=\bigoplus_{i \geq 1}\left(\mathbb{M}_{n_{i}}(\mathbb{C}) \otimes \mathcal{Z}_{i}\right) \supset$ $A q=\bigoplus_{i>1}\left(\mathbb{C}^{n_{i}} \otimes \mathcal{Z}_{i}\right)$. Let $i$ be such that $p \leq p_{i, j}$, for some $j \in\left\{1, \ldots, n_{i}\right\}$. Since $\mathbb{C}^{n_{i}} \otimes \mathcal{Z}_{i}$ is regular in $\mathbb{M}_{n_{i}}(\mathbb{C}) \otimes \mathcal{Z}_{i}$ and $\mathbb{C}^{n_{i}} \otimes \mathcal{Z}_{i}=A q_{i}$, for some $q_{i} \in \mathcal{P}(A q)$, we can find $u_{1}, \ldots, u_{m} \in \mathcal{N}_{M}(A)$ such that $\left(\mathbb{M}_{n_{i}}(\mathbb{C}) \otimes \mathcal{Z}_{i}\right)_{1} \subset \sum_{i=1}^{m}(A)_{1} u_{i}$. Since $p C \subset \mathbb{M}_{n_{i}}(\mathbb{C}) \otimes \mathcal{Z}_{i}$, we derive that $p w_{\lambda}^{*} \in \sum_{i=1}^{m}(A)_{1} u_{i}$, for all $\lambda \in \Lambda_{1}$. Combining this with (8.g), the definition of $\xi$ and the fact that $\eta$ normalizes $A$ gives that

$$
\begin{gathered}
p v_{\lambda}=\left(V_{\lambda} p\right)\left(p w_{\lambda}^{*}\right) \in \eta\left(\xi^{*} u_{\delta(\lambda)} \xi\right) \eta^{*} \sum_{i=1}^{m}(A)_{1} u_{i} \\
\subset|K| \sum_{k, k^{\prime} \in K} \sum_{i=1}^{m}(A)_{1} \eta u_{k} u_{\delta(\lambda)} u_{k^{\prime}} \eta^{*} u_{i}, \forall \lambda \in \Lambda_{1} .
\end{gathered}
$$

Recall that $N \subset q M q$ (unitally), $D \subset A q$ and $p \in A q$. Finally, let $\tilde{\Delta}=$ $\operatorname{Ad}\left(\eta^{*}\right)_{\mid N}: N \rightarrow M$. Since $\delta(\lambda)$ normalizes $K$ and $\eta \in \mathcal{N}_{M}(A)$, (8.h) implies that

$$
\left(\eta^{*} p \eta\right) \tilde{\Delta}\left(v_{\lambda}\right) \in|K| \sum_{k, k^{\prime} \in K} \sum_{i=1}^{m}(A)_{1} u_{\delta(\lambda)} u_{k k^{\prime}} \eta^{*} u_{i} \eta, \forall \lambda \in \Lambda_{1} .
$$

Since $\tilde{\Delta}(D) \subset A, \eta^{*} p \eta \leq \tilde{\Delta}\left(1_{N}\right)=\eta^{*} q \eta$ and $u_{k}(k \in K), \eta, u_{i}$ all normalize $A$, this completes the proof.

Proof of Theorem 8.1. Let $\Delta: N \rightarrow q M q$ be a unital $*$-homomorphism, for some $q \in \mathcal{P}(M)$, and identify $N$ with $\Delta(N)$. Assume that $N \nprec_{M} L(\Gamma)$. Since $D$ is regular in $N$ and $\sigma$ is mixing, by [33, Theorem 3.1 we get that $D \nprec_{M} L(\Gamma)$.

Further, we claim that we can find $u \in \mathcal{U}(M)$ such that $u L(\Lambda) u^{*} \subset L(\Gamma)$.

In case (a), this follows from Theorem 2.1, while in case (b) it follows from [39, Lemma 5.2 and [8], Theorem 3. By combining there two facts and Theorem 6.2 and by reasoning as in the proof of Theorem 8.2 (the torsion free case), the conclusion follows.

\section{9. $\mathrm{W}^{*}$-SUPERRIGIDITY}

In this section we prove Theorem A. Let us first restate it using the language of von Neumann algebras.

Theorem 9.1. Let $\Gamma$ be an ICC countable group which admits an infinite, almost normal subgroup $\Gamma_{0}$ such that the inclusion $\left(\Gamma_{0} \subset \Gamma\right)$ has the relative property $(T)$. Let $B$ be a non-trivial abelian von Neumann algebra. Denote $M=B^{\Gamma} \rtimes \Gamma$ and $A=B^{\Gamma}$.

Let $\rho: \Lambda \rightarrow \operatorname{Aut}(C)$ be a free ergodic action of a countable group $\Lambda$ on an abelian von Neumann algebra $C$. Denote $N=C \rtimes \Lambda$.

Let $\theta: N \rightarrow q M q$ be a $*$-isomorphism, for some projection $q \in M$.

Then $q=1$ and there exist a unitary $u \in M$, a character $\eta$ of $\Lambda$, and a group isomorphism $\delta: \Lambda \rightarrow \Gamma$ such that $\theta(C)=u A u^{*}$ and $\theta\left(v_{\lambda}\right)=\eta(\lambda) u u_{\delta(\lambda)} u^{*}$, for all $\lambda \in \Lambda$. 
Define the $*$-homomorphism $\Delta: N \rightarrow N \bar{\otimes} N$ by letting $\Delta\left(c v_{\lambda}\right)=c v_{\lambda} \otimes v_{\lambda}$, for all $c \in C$ and $\lambda \in \Lambda$. Note that $\Delta$ has been introduced in the proof of [42], Lemma 3.2 .

Since $N \cong q M q$, we can view $\Delta$ as a non-unital embedding of $M$ into $M \bar{\otimes} M$. In the proof of Theorem 9.1 we will apply Theorem 8.2 to $\Delta$. We will then need certain properties of $\Delta$, which we record in the following lemma.

Lemma 9.2. Let $Q \subset N$ be a von Neumann subalgebra. We have that:

(1) If $\Delta(Q) \prec_{N \bar{\otimes} N} N \otimes 1$, then $Q \prec_{N} C$.

(2) If $\Delta(Q) \prec_{N \bar{\otimes} N} 1 \otimes N$, then $Q$ is not diffuse.

(3) If $\Delta(N) \prec_{N \bar{\otimes} N} Q \bar{\otimes} N$, then $C \prec_{N} Q$.

(4) If $\Delta(N) \prec_{N \bar{\otimes} N} N \bar{\otimes} Q$, then $L(\Lambda) \prec_{N} Q$. Moreover, if $\Delta(N) s \prec_{N \bar{\otimes} N} N \bar{\otimes} Q$, for every non-zero projection $s \in \Delta(N)^{\prime} \cap N \bar{\otimes} N$, then $L(\Lambda) r \prec_{N} Q$, for every non-zero projection $r \in L(\Lambda)^{\prime} \cap N$.

Proof of Lemma 9.2. (1) Arguing by contradiction, if $Q \nprec_{N} C$, we can find a sequence $\left\{u_{n}\right\}_{n \geq 1} \subset \mathcal{U}(Q)$ such that $\left\|E_{C}\left(a u_{n} b\right)\right\|_{2} \rightarrow 0$, for all $a, b \in N$. We claim that

$$
\left\|E_{N \otimes 1}\left(x \Delta\left(u_{n}\right) y\right)\right\|_{2} \rightarrow 0, \text { for all } x, y \in N \bar{\otimes} N .
$$

Since $E_{N \otimes 1}$ is $N \otimes 1$-bimodular, we may assume that $x=1 \otimes a u_{g}, y=1 \otimes b u_{h}$, for some $a, b \in C$ and $g, h \in \Lambda$. Then for all $n \geq 1$, we have that

$$
x \Delta\left(u_{n}\right) y=\sum_{\lambda \in \Lambda} E_{C}\left(u_{n} v_{\lambda}^{*}\right) v_{\lambda} \otimes a v_{g} v_{\lambda} b v_{h} .
$$

Thus we get that $\left\|E_{N \otimes 1}\left(x \Delta\left(u_{n}\right) y\right)\right\|_{2}=\mid \tau\left(a \rho_{h^{-1}}(b) \mid\left\|E_{C}\left(u_{n} v_{\lambda}^{*}\right)\right\|_{2} \rightarrow 0\right.$, which proves (9.a). Since $u_{n} \in \mathcal{U}(Q)$, (9.a) implies that $\Delta(Q) \nprec_{N \bar{\otimes} N} N \otimes 1$, and we are done.

(2) The proof of this part is similar to the proof of (1), so we leave it as an exercise.

(3) If $\Delta(N) \prec_{N \bar{\otimes} N} Q \bar{\otimes} N$, then $\Delta(C) \prec_{N \bar{\otimes} N} Q \bar{\otimes} N$. The conclusion follows easily after noticing that $\Delta(C)=C \otimes 1$.

(4) Arguing by contradiction, assume that $L(\Lambda) r \nprec_{N} Q$, for some non-zero projection $r \in L(\Lambda)^{\prime} \cap N$. Since the group $\left\{v_{\lambda} r\right\}_{\lambda \in \Lambda}$ generates $L(\Lambda) r$ as a von Neumann algebra, we can find a sequence $\left\{\lambda_{n}\right\}_{n \geq 1} \subset \Lambda$ such that $\left\|E_{Q}\left(a v_{\lambda_{n}} r b\right)\right\|_{2} \rightarrow 0$, for all $a, b \in N$.

Define $s:=1 \otimes r \in \Delta(N)^{\prime} \cap N \bar{\otimes} N$. We claim that

$$
\left\|E_{N \bar{\otimes} Q}\left(x \Delta\left(v_{\lambda_{n}}\right) s y\right)\right\|_{2} \rightarrow 0, \forall x, y \in N \bar{\otimes} N .
$$

Since $E_{N \bar{\otimes} Q}$ is $N \otimes 1$-bimodular, we may take $x=1 \otimes a, y=1 \otimes b$, for some $a, b \in N$. Then $E_{N \bar{\otimes} Q}\left(x \Delta\left(v_{\lambda_{n}} s\right) y\right)=v_{\lambda_{n}} \otimes E_{Q}\left(a v_{\lambda_{n}} r b\right)$.

Since $\left\|E_{Q}\left(a v_{\lambda_{n}} r b\right)\right\|_{2} \rightarrow 0$, this proves (9.b). Now, since $\Delta\left(v_{\lambda_{n}}\right) s \in \mathcal{U}(\Delta(N) p)$, (9.b) implies that $\Delta(N) s \nprec_{N \bar{\otimes} N} N \bar{\otimes} Q$ and our proof by contradiction is over.

Remark. The moreover part of (4) was first noticed by Stefaan Vaes who pointed out to us that it can be used to simplify our initial proof of Theorem 9.1. Specifically, the moreover part of (4) enables us to avoid repeating the proof of Theorem 6.1 in the proof of Case (5) below.

Proof of Theorem 9.1. Let $\theta: N \rightarrow q M q$ be an isomorphism, for some $q \in \mathcal{P}(M)$. Let $t=\tau(q)^{-1}$ and $k \geq t$ be an integer. View $\mathbb{C}^{k}$ as the algebra of diagonal matrices in $\mathbb{M}_{k}(\mathbb{C})$ and let $r \in \mathbb{C}^{k} \otimes C$ be a projection of normalized trace $\frac{t}{k}$ in $\mathbb{M}_{k}(\mathbb{C}) \otimes C$. 
Consider the $*$-homomorphism $\operatorname{id}_{\mathbb{M}_{k}(\mathbb{C})} \otimes \Delta: \mathbb{M}_{k}(\mathbb{C}) \otimes N \rightarrow \mathbb{M}_{k}(\mathbb{C}) \otimes(N \bar{\otimes} N) \cong$ $\left(\mathbb{M}_{k}(\mathbb{C}) \otimes N\right) \bar{\otimes} N$. Since $\left(\mathrm{id}_{\mathbb{M}_{k}(\mathbb{C})} \otimes \Delta\right)(r)=r \otimes 1$ and $r\left(\mathbb{M}_{k}(\mathbb{C}) \otimes N\right) r \cong N^{t}$, we get a unital $*$-homomorphism $\Delta_{t}: N^{t} \rightarrow N^{t} \bar{\otimes} N$.

Fix an embedding $N \subset N^{t}$ and view $\Delta_{t}$ as a $*$-homomorphism $\Delta_{t}: N^{t} \rightarrow$ $N^{t} \bar{\otimes} N^{t}$. It is easy to see that Lemma 9.2 holds true if we replace $N$ and $\Delta$ by $N^{t}$ and $\Delta_{t}$, throughout. We denote by $C^{t}=r\left(\mathbb{C}^{k} \otimes C\right) r \subset N^{t}$. Observe that $C^{t} \subset N^{t}$ is a Cartan subalgebra and that $\Delta_{t}(x)=x \otimes q$, for all $x \in C^{t}$. For simplicity, we denote $1 \otimes q$ by $q$. From now on, we identify $N^{t} \cong M$ (via $\theta^{t}$, the $t$-amplification of $\theta$ ) and view $\Delta_{t}$ as a $*$-homomorphism $\Delta_{t}: M \rightarrow M \bar{\otimes} M$ with $\Delta_{t}(1)=q$.

To continue, assume for the moment that $(\diamond)$ there is a non-zero projection $s \in \Delta_{t}(M)^{\prime} \cap q(M \bar{\otimes} M) q$ such that $\Delta_{t}(M) s \nprec_{M \bar{\otimes} M} M \bar{\otimes} L(\Lambda)$. Let $\tilde{\Delta}_{t}: M \rightarrow M \bar{\otimes} M$ be given by $\tilde{\Delta}_{t}(x)=\Delta_{t}(x) s$. Since $\tilde{\Delta}_{t}(M) \nprec_{M \bar{\otimes} M} M \bar{\otimes} L(\Lambda)$, by applying Theorem 8.2 to $\tilde{\Delta}_{t}$ we conclude that we are in one of the following cases:

(1) There exist a finite index subgroup $\Gamma_{1}$ of $\Gamma$, a 1-1 map $\delta: \Gamma_{1} \rightarrow \Gamma \times \Gamma$, $u_{1}, \ldots, u_{n} \in \mathcal{N}_{M \bar{\otimes} M}(A \bar{\otimes} A)$ and $u \in \mathcal{U}(M \bar{\otimes} M)$ such that $\tilde{\Delta}:=\operatorname{Ad}(u) \circ \tilde{\Delta}_{t}: M \rightarrow$ $M \bar{\otimes} M$ satisfies

(a) $\tilde{\Delta}(A) \subset A \bar{\otimes} A$, there exists $0 \neq p \in \mathcal{P}(A \bar{\otimes} A)$ and $a_{i}^{\gamma} \in(A \bar{\otimes} A)_{1}$, for all $i \in$ $\{1, \ldots, n\}, \gamma \in \Gamma_{1}$ such that $p \leq \tilde{\Delta}(1)$ and $p \tilde{\Delta}\left(u_{\gamma}\right)=\sum_{i=1}^{n} a_{i}^{\gamma} u_{\delta(\gamma)} u_{i}$, for all $\gamma \in \Gamma_{1}$, and

(b) there is a finite subgroup $K$ of $\Gamma \times \Gamma$ such that $\delta\left(\gamma \gamma^{\prime}\right)^{-1} \delta(\gamma) \delta\left(\gamma^{\prime}\right) \in K$ and $\delta(\gamma)$ normalizes $K$, for all $\gamma, \gamma^{\prime} \in \Gamma_{1}$.

(2) $\tilde{\Delta}_{t}\left(L\left(\Gamma_{0}\right)\right) \prec_{M \bar{\otimes} M} L(\Gamma) \otimes 1$.

(3) $\tilde{\Delta}_{t}\left(L\left(\Gamma_{0}\right)\right) \prec_{M \bar{\otimes} M} 1 \otimes L(\Gamma)$.

(4) $\tilde{\Delta}_{t}(M) \prec_{M \bar{\otimes} M} L(\Gamma) \bar{\otimes} M$.

On the other hand, if $(\diamond)$ fails, then we are in the following case:

(5) $\Delta_{t}(M) s \prec_{M \bar{\otimes} M} M \bar{\otimes} L(\Gamma)$, for any non-zero projection $s \in \Delta_{t}(M)^{\prime} \cap q(M \bar{\otimes} M) q$.

For the rest of the proof, we analyze each one of these cases and show that they either lead to a contradiction or imply that $(\star) C^{t} \prec_{M} A$ or that $(\star \star) u L(\Lambda) u^{*} \subset$ $L(\Gamma)$, for some $u \in \mathcal{U}(M)$. If $(\star)$ holds, since $C^{t}$ and $A$ are Cartan subalgebras of $N^{t} \cong M$, 35, Theorem A.1 provides a unitary $v \in M$ such that $A=v C^{t} v^{*}$.

Popa's cocycle/OE superrigidity theorems [37, 5.2 and 5.6 then imply that $t=1$ (thus $q=1$ ) and that the isomorphism $\theta: N \rightarrow M$ is of the desired form. If $(\star \star)$ holds, then the conclusion follows from 34, Theorem 0.7 (see Theorem 6.3).

Case (1). Let $G$ be the set of all $g \in \Gamma_{1}$ for which we can find $k \in K$ such that $\delta(g) k \in \Gamma \times\{e\}$. Since $\delta$ satisfies (b), $G$ is a subgroup of $\Gamma_{1}$.

Let us prove that $G$ is finite. If $g \in G$, then $\delta(g) \in(\Gamma \times\{e\}) K$. Condition (a) implies that $p \tilde{\Delta}\left(u_{g}\right) \in \sum_{i=1}^{n} \sum_{k \in K}(M \bar{\otimes} A)_{1} u_{k} u_{i}$. This shows in particular that $\tilde{\Delta}(L(G)) \prec_{M \bar{\otimes} M} M \bar{\otimes} A$. On the other hand, we may assume that (2) and (3) are false since these cases will be dealt with later. Since the inclusion $\tilde{\Delta}\left(L\left(\Gamma_{0}\right)\right) \subset$ $\tilde{\Delta}(L(\Gamma))$ is rigid and quasi-regular, by Theorem 2.1 we can find a unitary $v \in M \bar{\otimes} M$ such that $\tilde{\Delta}(L(G)) \subset \tilde{\Delta}(L(\Gamma)) \subset v(L(\Gamma) \bar{\otimes} L(\Gamma)) v^{*}$. The last two facts readily imply that $\tilde{\Delta}(L(G)) \prec_{M \bar{\otimes} M} L(\Gamma) \otimes 1$.

By the construction of $\tilde{\Delta}$ it follows that $\Delta_{t}(L(G)) \prec_{M \bar{\otimes} M} L(\Gamma) \otimes 1$. But then Lemma 9.2 (1) (which holds for $\Delta_{t}$ ) yields that $L(G) \prec_{M} C^{t}$.

Further, by [45], Lemma 3.5 we deduce that $C^{t} \prec_{M} L(G)^{\prime} \cap M$. If $G$ is infinite, then $L(G)$ is diffuse. Hence, since $\sigma$ is mixing, 33, Theorem 3.1 implies that 
$L(G)^{\prime} \cap M \subset L(\Gamma)$. Thus, we would get that $C^{t} \prec_{M} L(\Gamma)$. Since $C^{t}$ is regular in $M$, by applying [33], Theorem 3.1 (see also Lemma 3.4) we derive a contradiction. This shows that $G$ is finite.

Using the fact that $G$ is finite we next deduce the following claim:

Claim. Let $\left\{x_{m}\right\}_{m \geq 1} \subset M$ be a sequence with $\left\|x_{m}\right\| \leq 1$. Denote $x_{m}^{\gamma}=E_{A}\left(x_{m} u_{\gamma}^{*}\right)$ and assume that $\left\|x_{m}^{\gamma}\right\|_{2} \rightarrow 0$, for all $\gamma \in \Gamma$. Then we have that

$$
\left\|E_{M \otimes 1}\left(y p \tilde{\Delta}\left(x_{m}\right) z\right)\right\|_{2} \rightarrow 0 \text {, for all } y, z \in M \bar{\otimes} M \text {. }
$$

Proof of the Claim. Let $M_{1}=A \rtimes \Gamma_{1}$. Since $\Gamma_{1}$ has finite index in $\Gamma$, we may assume that $x_{m} \in M_{1}$, for all $m \geq 1$.

Since $E_{M \otimes 1}$ is $M \otimes 1$-bimodular, we may assume that $y=1 \otimes a u_{g}$ and $z=1 \otimes b u_{h}$, for $a, b \in A$ and $g, h \in \Gamma$. Then $E_{M \otimes 1}(y x z)=E_{M \otimes 1}\left(\left(1 \otimes a \sigma_{g}(b)\right) x\left(1 \otimes u_{g h}\right)\right.$ for all $x \in M \bar{\otimes} M$. Thus, to prove (9.c), we may assume that $y \in A \bar{\otimes} A$ and $z=u_{g}$, for some $g \in \Gamma \times \Gamma$. Since

$$
\left\|E_{M \otimes 1}\left(y p \tilde{\Delta}\left(x_{m}\right) u_{g}\right)\right\|_{2} \leq\left\|E_{M \bar{\otimes} A}\left(y p \tilde{\Delta}\left(x_{m}\right) u_{g}\right)\right\|_{2} \leq\|y\|\left\|E_{M \bar{\otimes} A}\left(p \tilde{\Delta}\left(x_{m}\right) u_{g}\right)\right\|_{2}
$$

(here we use that $y \in M \bar{\otimes} A$ ), it is sufficient to prove that

$$
\left\|E_{M \bar{\otimes} A}\left(p \tilde{\Delta}\left(x_{m}\right) u_{g}\right)\right\|_{2} \rightarrow 0 \text {, for all } g \in \Gamma \times \Gamma .
$$

Now, (a) gives that

$$
p \tilde{\Delta}\left(x_{m}\right)=\sum_{\gamma \in \Gamma_{1}} \tilde{\Delta}\left(x_{m}^{\gamma}\right) p \tilde{\Delta}\left(u_{\gamma}\right)=\sum_{i=1}^{n} \sum_{\gamma \in \Gamma_{1}} \tilde{\Delta}\left(x_{m}^{\gamma}\right) a_{i}^{\gamma} u_{\delta(\gamma)} u_{i} .
$$

As $\tilde{\Delta}\left(x_{m}^{\gamma}\right) a_{i}^{\gamma} \in A \bar{\otimes} A$, this identity implies that in order to get (9.d) it suffices to show that

$$
\text { \| } \sum_{\gamma \in \Gamma_{1}} \tilde{\Delta}\left(x_{m}^{\gamma}\right) a_{i}^{\gamma} E_{M \bar{\otimes} A}\left(u_{\delta(\gamma)} u_{i} u_{g}\right) \|_{2} \rightarrow 0, \text { for all } i \in\{1, \ldots, n\} .
$$

To prove (9.e), fix $i \in\{1, \ldots, n\}$ and denote $v=u_{i} u_{g}$. Since $u_{\delta(\gamma)} v$ normalizes $A \bar{\otimes} A$ we can find $p_{\gamma} \in \mathcal{P}(A \bar{\otimes} A)$ such that $E_{M \bar{\otimes} A}\left(u_{\delta(\gamma)} v\right)=p_{\gamma} u_{\delta(\gamma)} v$. Since $\delta$ is 1-1, the $A \bar{\otimes} A$-bimodules $\left\{(A \bar{\otimes} A) u_{\delta(\gamma)} v\right\}_{\gamma \in \Gamma_{1}}$ are mutually orthogonal.

Altogether, (9.e) can be rewritten as

$$
\sum_{\gamma \in \Gamma_{1}} \tau\left(\left|\tilde{\Delta}\left(x_{m}^{\gamma}\right)\right|^{2}\left|a_{i}^{\gamma}\right|^{2} p_{\gamma}\right) \rightarrow 0, \text { as } m \rightarrow \infty .
$$

Next, we claim that $\sum_{\gamma \in \Gamma_{1}} \tau\left(p_{\gamma}\right)<\infty$. Identify $A=L^{\infty}(X)$, where $(X, \mu)$ is a probability space. Then $\sigma$ induces a free ergodic p.m.p. action $\Gamma \curvearrowright X$. On $\left(X^{2}, \mu^{2}\right):=(X \times X, \mu \times \mu)$ we consider the direct product action of $\Gamma \times \Gamma$ : $\left(\gamma_{1}, \gamma_{2}\right) \circ\left(x_{1}, x_{2}\right)=\left(\gamma_{1} x_{1}, \gamma_{2} x_{2}\right)$. Let $\phi$ be the automorphism of $X^{2}$ given by $v a v^{*}=a \circ \phi^{-1}$, for all $a \in L^{\infty}\left(X^{2}\right)$. Then $p_{\gamma}$ is precisely the characteristic function of the set of $x \in X^{2}$ satisfying $(\delta(\gamma) \circ \phi)^{-1}(x) \in(\Gamma \times\{e\}) x$.

Hence, if we let $Y_{\gamma}=\left\{x \in X^{2} \mid \phi^{-1}(x) \in(\Gamma \times\{e\}) \delta(\gamma) x\right\}$, then $\tau\left(p_{\gamma}\right)=\mu^{2}\left(Y_{\gamma}\right)$. If $\gamma, \gamma^{\prime} \in \Gamma$ satisfy $\mu^{2}\left(Y_{\gamma} \cap Y_{\gamma^{\prime}}\right)>0$, then the freeness of the action $\Gamma \times \Gamma \curvearrowright X^{2}$ implies that $(\Gamma \times\{e\}) \delta(\gamma) \cap(\Gamma \times\{e\}) \delta\left(\gamma^{\prime}\right) \neq \emptyset$. Thus $\delta(\gamma) \delta\left(\gamma^{\prime}\right)^{-1} \in \Gamma \times\{e\}$; hence $\gamma \gamma^{\prime-1} \in G$. This shows that if $G \delta(\gamma) \cap G \delta\left(\gamma^{\prime}\right)=\emptyset$, then $\mu^{2}\left(Y_{\gamma} \cap Y_{\gamma^{\prime}}\right)=0$. Since $\delta$ is 1-1, it follows that $\sum_{\gamma \in \Gamma} \mu^{2}\left(Y_{\gamma}\right) \leq|G|$; hence $\sum_{\gamma \in \Gamma} \tau\left(p_{\gamma}\right)<\infty$, as claimed.

To prove (9.f) and the claim, fix $\varepsilon>0$ and let $F \subset \Gamma_{1}$ be finite such that $\sum_{\gamma \in \Gamma_{1} \backslash F} \tau\left(p_{\gamma}\right)<\varepsilon$. 
Since $\left\|x_{m}^{\gamma}\right\|,\left\|a_{i}^{\gamma}\right\|,\left\|p_{\gamma}\right\| \leq 1$ and $\tau\left(\left|\tilde{\Delta}\left(x_{m}^{\gamma}\right)\right|^{2}\right)=\left\|\tilde{\Delta}\left(x_{m}^{\gamma}\right)\right\|_{2}^{2} \leq\left\|x_{m}^{\gamma}\right\|_{2}^{2}$, we get that

$$
\sum_{\gamma \in \Gamma_{1}} \tau\left(\left|\tilde{\Delta}\left(x_{m}^{\gamma}\right)\right|^{2}\left|a_{i}^{\gamma}\right|^{2} p_{\gamma}\right) \leq \varepsilon+\sum_{\gamma \in F}\left\|x_{m}^{\gamma}\right\|_{2}^{2} .
$$

Since $\left\|x_{m}^{\gamma}\right\|_{2} \rightarrow 0$ and $\varepsilon>0$ is arbitrary, this indeed proves (9.f).

Recall that $\tilde{\Delta}(x)=u \Delta_{t}(x) s u^{*}$, for all $x \in M$, and that $\Delta_{t}(x)=(x \otimes 1) q$, for all $x \in C^{t}$. Since $p \leq \tilde{\Delta}(1)=u s u^{*}$, it follows that $u^{*} p \tilde{\Delta}(x) u=u^{*} p u(x \otimes 1)$ and hence that $\left\|u^{*} p \tilde{\Delta}(x) u\right\|_{2}=\|p\|_{2}$, for all $x \in \mathcal{U}\left(C^{t}\right)$. Then the above claim implies that we cannot find a sequence $\left\{x_{m}\right\}_{m \geq 1} \subset \mathcal{U}\left(C^{t}\right)$ such that $\left\|E_{A}\left(x_{m} u_{\gamma}^{*}\right)\right\|_{2} \rightarrow 0$, for all $\gamma \in \Gamma$.

Thus $C^{t} \prec_{M} A$, which settles Case (1).

Case (2). If $\tilde{\Delta}_{t}\left(L\left(\Gamma_{0}\right)\right) \prec_{M \bar{\otimes} M} L(\Gamma) \otimes 1$, then $\Delta_{t}\left(L\left(\Gamma_{0}\right)\right) \prec_{M \bar{\otimes} M} L(\Gamma) \otimes 1$ and Lemma 9.2 (1) implies that $L\left(\Gamma_{0}\right) \prec_{M} C^{t}$. Since $\Gamma_{0}$ is infinite, we derive a contradiction by arguing as the proof of (1).

Case (3). If $\tilde{\Delta}_{t}\left(L\left(\Gamma_{0}\right)\right) \prec_{M \bar{\otimes} M} 1 \otimes L(\Gamma)$, then $\Delta_{t}\left(L\left(\Gamma_{0}\right)\right) \prec_{M \bar{\otimes} M} 1 \otimes L(\Gamma)$ and Lemma $9.2(2)$ implies that $L\left(\Gamma_{0}\right)$ cannot be diffuse. This contradicts the fact that $\Gamma_{0}$ is infinite.

Case (4). If $\tilde{\Delta}_{t}(M) \prec_{M \bar{\otimes} M} L(\Gamma) \bar{\otimes} M$, then $\Delta_{t}(M) \prec_{M \bar{\otimes} M} L(\Gamma) \bar{\otimes} M$ and Lemma 9.1 (3) entails $C^{t} \prec_{M} L(\Gamma)$. Since $\sigma$ is mixing and $C^{t}$ is regular in $M$, 33, Theorem 3.1 leads to a contradiction.

Case (5). Assume that $\Delta_{t}(M) s \prec_{M \bar{\otimes} M} M \bar{\otimes} L(\Gamma)$, for any non-zero projection $s \in \Delta_{t}(M)^{\prime} \cap q(M \bar{\otimes} M) q$. Then the moreover part of Lemma 9.2 (4) gives that $L(\Lambda) r \prec_{M} L(\Gamma)$, for any non-zero projection $r \in L(\Lambda)^{\prime} \cap N$. Since $\Gamma$ is ICC, by reasoning as in the end of the proof of Theorem 2.1 we can find a unitary $u \in M$ such that $u L(\Lambda) u^{*} \subset \Gamma$.

This finishes the proof of the last case of Theorem 9.1.

\section{FURTHER APPLICATIONS}

In this section, we derive several applications of the results of Section 8 .

(I) Group von Neumann algebra decomposition. First, we use Theorem 8.2 to provide a large class of $\mathrm{II}_{1}$ factors which cannot be decomposed as the group von Neumann algebra, $L(G)$, of some countable group $G$.

Corollary 10.1. Let $\Gamma$ be a countable ICC group which admits an infinite, almost normal subgroup $\Gamma_{0}$ such that the inclusion $\left(\Gamma_{0} \subset \Gamma\right)$ has the relative property $(T)$. Assume that $\sup \{|K| \mid K$ is a finite subgroup of $\Gamma\}$ is finite. Let $l=l(\Gamma)$ be the least common multiple of $|K|$, with $K$ ranging over all finite subgroups of $\Gamma$.

Let $B$ be a non-trivial abelian von Neumann algebra. Set $M=B^{\Gamma} \rtimes_{\sigma} \Gamma$ and let $p \in \mathcal{P}(M)$.

If $\tau(p) \notin\left\{\frac{1}{l}, \frac{2}{l}, \ldots, \frac{l}{l}\right\}$, then the $\mathrm{II}_{1}$ factor $p M p$ is not isomorphic to a group von Neumann algebra. In particular, if $\Gamma$ is torsion free (i.e. $l=1)$, then $p M p$ is not isomorphic to a group von Neumann algebra, for any $p \neq 1$.

Proof. Assume that $p M p \cong N:=L(G)$, for some projection $p \in M$ and a countable (necessarily) ICC group $G$. In other words, we have that $M \cong N^{t}$, where $t=$ $\tau(p)^{-1}$.

Next, let $\Delta: N \rightarrow N \bar{\otimes} N$ be the $*$-homomorphism given by $\Delta\left(u_{g}\right)=u_{g} \otimes u_{g}$, for all $g \in G$. By amplifying $\Delta$ (as in the proof of Theorem 9.1), we get a $*-$ homomorphism $\Delta_{t}: N^{t} \rightarrow N^{t} \bar{\otimes} N^{t}$ such that $\tau\left(\Delta_{t}(1)\right)=\tau(p)$. We continue with: 
Lemma 10.2. Let $Q \subset N^{t}$ be a not necessarily unital von Neumann subalgebra. Then we have the following:

(1) If $\Delta_{t}(Q) \prec_{N^{t} \bar{\otimes} N^{t}} N^{t} \otimes 1$ or $\Delta_{t}(Q) \prec_{N^{t} \bar{\otimes} N^{t}} 1 \otimes N^{t}$, then $Q$ cannot be diffuse.

(2) If $\Delta_{t}\left(N^{t}\right) \prec_{N^{t} \bar{\otimes} N^{t}} Q \bar{\otimes} N^{t}$ or $\Delta_{t}\left(N^{t}\right) \prec_{N^{t} \bar{\otimes} N^{t}} N^{t} \bar{\otimes} Q$, then $N^{t} \prec_{N^{t}} Q$.

The proof of this lemma is analogous to the proof of Lemma 9.1 and we therefore leave it as an exercise.

Now, since $M \cong N^{t}$, we can view $\Delta_{t}$ as a $*$-homomorphism $\Delta_{t}: M \rightarrow M \bar{\otimes} M$. Thus, we can apply Theorem 8.2 to $\Delta_{t}$. Lemma 10.2 guarantees that conditions (1)-(2) from 8.2 are satisfied. Finally, by the last assertion of Theorem 8.2 we deduce that $\tau(p)=\tau\left(\Delta_{t}(1)\right) \in l^{-1} \mathbb{N}$, as claimed.

Remarks 10.3. (1) Recall that $A \curlywedge \Gamma=\left(\bigoplus_{\Gamma} A\right) \rtimes \Gamma$, where $\Gamma$ acts on $\bigoplus_{\Gamma} A$ by shifting the copies of $A$, is the wreath product of two groups $A$ and $\Gamma$. Corollary 10.1 implies that if $n \geq 3$ and $t \in(0,1) \backslash \mathbb{Q}$, then $L\left(\mathbb{Z} \succ \mathrm{SL}_{n}(\mathbb{Z})\right)^{t}$ is not a group von Neumann algebra. Indeed, $\mathrm{SL}_{n}(\mathbb{Z})$ is ICC and has property $(\mathrm{T})$ by Kazhdan's classical result (20]). Additionally, if $m \geq 3$, then the kernel of the natural quotient $\mathrm{SL}_{n}(\mathbb{Z}) \rightarrow$ $\mathrm{SL}_{n}(\mathbb{Z} / m \mathbb{Z})$ is a torsion free group. This shows that $|K| \leq\left|\mathrm{SL}_{n}(\mathbb{Z} / m \mathbb{Z})\right|$, for every finite subgroup $K$ of $\mathrm{SL}_{n}(\mathbb{Z})$.

(2) The first examples of $\mathrm{II}_{1}$ factors which are not group von Neumann algebras were exhibited by Connes by means of his $\chi(M)$ invariant (4 4$)$. Recently, Popa's deformation/rigidity theory has been used to give new examples of such factors ([17, 40]). In all of these cases, one moreover proves that the $\mathrm{II}_{1}$ factors involved do not have anti-automorphisms, i.e. $M \neq M^{o p}$.

(3) If $M$ is as in Corollary 10.1, then $p M p$ admits an involutary anti-automorphism, for every $p \in \mathcal{P}(M)$. Indeed, the formula $\Phi\left(a u_{\gamma}\right)=u_{\gamma^{-1}} a$, for $a \in B^{\Gamma}$ and $\gamma \in \Gamma$, defines an anti-automorphism of $M$. If we take $p \in B^{\Gamma}$, then $\Phi(p)=p$ and $\Phi_{p}=\Phi_{\mid p M p}$ is an involutary anti-automorphism of $p M p$.

The first examples of $\mathrm{II}_{1}$ factors which are not group von Neumann algebras and yet have anti-automorphisms have been constructed in [18]. We point out that, as opposed to our examples, the examples constructed in [18] have no involutary anti-automorphism.

(II) Symmetry groups of $\mathbf{I I}_{1}$ factors. Given a $\mathrm{II}_{1}$ factor $M$, there are three natural objects capturing the symmetries of $M$ :

$\mathcal{F}(M)$, the fundamental group of $M$, Out $(M)$, the outer automorphism group of $M$ and $\operatorname{Bimod}(M)$, the set of bi-finite Hilbert $M$-bimodules $\mathcal{H}$, i.e. such that $\operatorname{dim}\left({ }_{M} \mathcal{H}\right), \operatorname{dim}\left(\mathcal{H}_{M}\right)<\infty$.

The calculation of these invariants is an extremely challenging problem and it is only recently that explicit calculations were obtained for large families of $\mathrm{II}_{1}$ factors by using Popa's deformation/rigidity theory. While there is, by now, an extensive literature on this problem (see e.g. the introduction of [45]), we only mention here that the existence of $\mathrm{II}_{1}$ factors $M$ for which $\mathcal{F}(M)$, $\operatorname{Out}(M)$ and $\operatorname{Bimod}(M)$ are trivial has been proven in [33, [17] and [46], respectively.

In this subsection, we consider three natural invariants for $\mathrm{II}_{1}$ factors which generalize the ones from above.

Definition 10.4. Let $M$ be a $\mathrm{II}_{1}$ factor. Then we define

- $\mathcal{F}_{s}(M)$, the fundamental semigroup of $M$, to be the set of $t>0$ for which there exists a unital $*$-homomorphism $\theta: M \rightarrow M^{t}$. 
- $\operatorname{End}(M)$, the endomorphism semigroup of $M$, to be the set of unital $*$-homomorphisms $\theta: M \rightarrow M$, and

- $\operatorname{LFBimod}(M)$ to be the set of left-finite Hilbert $M$-bimodules $\mathcal{H}$, i.e. such that $\operatorname{dim}(M \mathcal{H})<\infty$.

It is clear that $\mathcal{F}_{s}(M)$ is a semigroup (with respect to multiplication) which contains $\mathcal{F}(M)$. Thus, whenever $\mathcal{F}(M)=(0,+\infty)$ (e.g. if $M$ is the hyperfinite $\mathrm{II}_{1}$ factor), we get that $\mathcal{F}_{s}(M)=(0,+\infty)$. Also, we have that $\mathbb{N} \subset \mathcal{F}_{s}(M)$. Furthermore, we have a dichotomy: either $\mathcal{F}_{s}(M) \cap(0,1)=\emptyset$ or $\mathcal{F}_{s}(M)=(0,+\infty)$. This is a consequence of the following two facts: $(\star)$ if $\left\{t_{n}\right\}_{n \geq 1} \in \mathcal{F}_{s}(M)$ is a sequence such that $t:=\sum_{n \geq 1} t_{n}<\infty$, then $t \in \mathcal{F}_{s}(M)$, and $(\star \star)$ if $s \in(0,1)$, then every $t>0$ can be written as $t=\sum_{n>1} t_{n}$, with $t_{n}$ being a power of $s$, for all $n$.

Let us elaborate on the definition of $\operatorname{LFBimod}(M)$. Any left-finite Hilbert $M$ bimodule $\mathcal{H}$ comes from a unital embedding of $M$ into one of its amplifications (5); see also [32]). Indeed, if $t=\operatorname{dim}_{M}(\mathcal{H})$, then $M^{\prime} \cap \mathbb{B}(\mathcal{H}) \cong\left(M^{o p}\right)^{t}$ and thus we obtain a unital $*$-homomorphism $\theta: M \rightarrow M^{t}$. Conversely, any unital $*$-homomorphism $\theta: M \rightarrow M^{t}$ induces a left-finite $M$-bimodule, which we denote by $\mathcal{H}_{\theta}$. For this, first represent $M^{t}=p\left(\mathbb{M}_{n}(C) \otimes M\right) p$, where $n \geq t$ is an integer and $p \in \mathbb{M}_{n}(\mathbb{C}) \otimes M$ has normalized trace equal to $\frac{t}{n}$. Then set $\mathcal{H}_{\theta}=\left(\mathbb{M}_{1, n}(\mathbb{C}) \otimes L^{2}(M)\right) p$ and define the left and right module actions by $x \cdot \xi=x \xi$ and $\xi \cdot x=\xi \theta(x)$.

Note also that if $\mathcal{H}$ and $\mathcal{K}$ are two left-finite $M$-bimodules, then so is their Connes tensor product, $\mathcal{H} \otimes_{M} \mathcal{K}$ (see [32], 1.3.4).

As a consequence of Theorem 8.1 and of results from 28 we obtain the first (partial) calculations of these invariants. We refer the reader to [28, 2.4. for the definition of the complete metric approximation property (abbreviated CMAP).

Corollary 10.5. Let $\Gamma_{1}, \Gamma_{2}$ be two ICC, torsion free groups, with $\Gamma_{2}$ non-amenable. Assume that $L\left(\Gamma_{1}\right), L\left(\Gamma_{2}\right)$ have the CMAP (e.g. $\Gamma_{1}=\mathbb{F}_{m}, \Gamma_{2}=\mathbb{F}_{n}$, for $2 \leq m, n \leq$ $\infty)$ and set $\Gamma=\Gamma_{1} \times \Gamma_{2}$.

Let $B$ be a non-trivial abelian von Neumann algebra. Let $\sigma: \Gamma \rightarrow \operatorname{Aut}\left(B^{\Gamma}\right)$ be the Bernoulli action. Denote $M=B^{\Gamma} \rtimes \Gamma$ and $A=B^{\Gamma}$.

If $\theta: M \rightarrow M^{t}$ is a unital $*$-homomorphism for some $t \leq 1$, then $t=1$. Moreover, there exist a character $\eta$ of $\Gamma$, an injective group morphism $\delta: \Gamma \rightarrow \Gamma$ and a unitary $u \in M$ such that $\theta(A) \subset u A u^{*}$ and $\theta\left(u_{\gamma}\right)=\eta(\gamma) u u_{\delta(\gamma)} u^{*}$, for all $\gamma \in \Gamma$. In particular, every $\theta \in \operatorname{End}(M)$ is irreducible, i.e. $\theta(M)^{\prime} \cap M=\mathbb{C} 1$.

In other words, if $\mathcal{H}$ is a Hilbert $M$-bimodule with $\operatorname{dim}_{M}(\mathcal{H}) \leq 1$, then we must have that $\operatorname{dim}_{M}(\mathcal{H})=1$. Equivalently, $\mathcal{F}_{s}(M) \cap(0,1)=\emptyset$, i.e. $M$ does not embed into $M^{t}$, for $t<1$. Moreover, Corollary 10.5 gives a description of $\operatorname{End}(M)$. Roughly speaking, every endomorphism $\theta: M \rightarrow M$ is determined by the following data:

- a character $\eta$ of $\Gamma$, a group embedding $\delta: \Gamma \rightarrow \Gamma$ and

- a "realization" of $\sigma$ as a quotient of $\sigma_{\mid \delta(\Gamma)}$.

In view of Corollary 10.5, it seems reasonable to conjecture that there are $\mathrm{II}_{1}$ factors $M$ for which $\operatorname{LFBimod}(M)$ is trivial, i.e. such that every left-finite Hilbert $M$-bimodule is isomorphic to $L^{2}(M) \otimes \ell_{n}^{2}$, for some $n \geq 1$, where $\ell_{n}^{2}$ is the Hilbert space of dimension $n$. In this case, we would necessarily have that $\mathcal{F}_{s}(M)=\mathbb{N}$ and $\operatorname{End}(M)=\operatorname{Int}(M)$. To support our conjecture, we notice below that, for certain $\mathrm{II}_{1}$ factors $M, \operatorname{LFBimod}(M)$ is "countably generated" (see Remark 10.8). 
Proof of Corollary 10.5. By Theorem 8.1 (applied to the situation $N=M$ ) we only need to argue that $\theta(M) \nprec_{M} L(\Gamma)$. If this were not the case, then, since $L(\Gamma)$ has the CMAP and $M$ is a factor, we would get that $M \cong \theta(M)$ also has the CMAP. Notice that the restriction of $\sigma$ to $\Gamma_{2}$ can be seen as the Bernoulli action of $\Gamma_{2}$ on $\tilde{B}^{\Gamma_{2}}$, where $\tilde{B}=B^{\Gamma_{1}}$. Since $\Gamma_{1}$ is infinite we have that $\tilde{B} \cong L(\mathbb{Z})$ and thus $M \supset B^{\Gamma} \rtimes_{\sigma_{\mid \Gamma_{2}}} \Gamma_{2} \cong L\left(\mathbb{Z} \imath \Gamma_{2}\right)$. Altogether, we would derive that $L\left(\mathbb{Z} \imath \Gamma_{2}\right)$ has the CMAP. Since $\Gamma_{2}$ is non-amenable, this contradicts 28, Corollary 2.12.

The irreducibility assertion follows easily from the first part.

(III) Group measure space decompositions of rigid factors. In this section, we consider $\mathrm{II}_{1}$ factors $M$ which have property $(\mathrm{T})$, in the sense of Connes-Jones 10. Our main goal is to prove that $M$ admits at most countably many group measure space decompositions (see Theorem 10.7). We start with the following lemma:

Lemma 10.6. Let $M$ and $\mathcal{M}$ be two separable $I I_{1}$ factors, with $M$ having property (T). Let $\left\{\Delta_{i}: M \rightarrow \mathcal{M} \mid i \in I\right\}$ be an uncountable family of not necessarily unital *-homomorphisms.

- Then there exists an uncountable subset $J$ of I such that for every $i, j \in I$ we can find $0 \neq v \in \mathcal{M}$ satisfying $v=\Delta_{i}(1) v \Delta_{j}(1)$ and $\Delta_{i}(x) v=v \Delta_{j}(x)$, for all $x \in M$.

- Assume moreover that $\Delta_{i}$ is irreducible (i.e. $\Delta_{i}(M)^{\prime} \cap \Delta_{i}(1) \mathcal{M} \Delta_{i}(1)=\mathbb{C} \Delta_{i}(1)$ ), for every $i \in I$. Then for all $i, j \in J$ we have that $\tau\left(\Delta_{i}(1)\right)=\tau\left(\Delta_{j}(1)\right)$ and that $\Delta_{i}=A d(u) \circ \Delta_{j}$, for some $u \in \mathcal{U}(\mathcal{M})$. In particular, the set $\left\{\tau\left(\Delta_{i}(1)\right) \mid i \in I\right\}$ is countable.

This lemma is proven by a separability argument going back to [6] (see also [32]). For completeness, let us sketch its proof.

Proof. Let $\varepsilon \in\left(0, \frac{1}{4}\right)$. After replacing $I$ with an uncountable subset, we can assume that $\tau\left(\Delta_{i}(1)\right) \in((1-\varepsilon) t, t)$, for all $i \in I$, for some $t \in(0,1)$. Let $p \in \mathcal{M}$ be a projection of trace $t$. Since $\tau\left(\Delta_{i}(1)\right) \leq \tau(p)$, we may assume that $\Delta_{i}(1) \leq p$, for all $i \in I$.

Next, for every $i, j \in I$, let $\mathcal{H}_{i, j}=L^{2}\left(\Delta_{i}(1) \mathcal{M} \Delta_{j}(1)\right)$ and $\xi_{i, j}=\Delta_{i}(1) \Delta_{j}(1) \in$ $\mathcal{H}_{i, j}$. Endow $\mathcal{H}_{i, j}$ with the Hilbert $M$-bimodule structure given by $x \cdot \xi \cdot y=$ $\Delta_{i}(x) \xi \Delta_{j}(y)$. Then for every $x \in \mathcal{U}(M)$, we have that

$$
\begin{aligned}
\left\|x \cdot \xi_{i, j}-\xi_{i, j} \cdot x\right\| & =\left\|\Delta_{i}(x) \Delta_{j}(1)-\Delta_{i}(1) \Delta_{j}(x)\right\|_{2} \\
& \leq\left\|\Delta_{i}(x)-\Delta_{j}(x)\right\|_{2}+\left\|\Delta_{i}(1)-p\right\|_{2}+\left\|\Delta_{j}(1)-p\right\|_{2} \\
& \leq 2 \sqrt{\varepsilon t}+\left\|\Delta_{i}(x)-\Delta_{j}(x)\right\|_{2} .
\end{aligned}
$$

Also, it is easy to see that $\left\|\xi_{i, j}\right\| \geq \sqrt{t}(1-2 \sqrt{\varepsilon})>0$. Thus, we have that

$$
\frac{\left\|x \cdot \xi_{i, j}-\xi_{i, j} \cdot x\right\|}{\left\|\xi_{i, j}\right\|} \leq \frac{2 \sqrt{\varepsilon}}{1-2 \sqrt{\varepsilon}}+\frac{\left\|\Delta_{i}(x)-\Delta_{j}(x)\right\|_{2}}{\sqrt{t}(1-2 \sqrt{\varepsilon})}, \forall x \in \mathcal{U}(M) .
$$

Since $L^{2}(\mathcal{M})$ is a separable Hilbert space, this estimate implies that for every finite set $F \subset \mathcal{U}(M)$ we can find an uncountable set $J \subset I$ such that $\frac{\left\|x \cdot \xi_{i, j}-\xi_{i, j} \cdot x\right\|}{\left\|\xi_{i, j}\right\|} \leq$ $\frac{3 \sqrt{\varepsilon}}{1-2 \sqrt{\varepsilon}}$, for all $x \in F$ and every $i, j \in J$. Thus, if we choose $\varepsilon$ small enough, then property $(\mathrm{T})$ guarantees that $\mathcal{H}_{i, j}$ has a central vector, for every $i \neq j \in J([10$, [35]). In other words, we can find $v \in \Delta_{i}(1) \mathcal{M} \Delta_{j}(1)$ such that $\Delta_{i}(x) v=v \Delta_{j}(x)$, for all $x \in M$. This proves the first assertion. 
The second assertion is immediate once we notice that $v v^{*} \in \Delta_{i}(M)^{\prime} \cap$ $\Delta_{i}(1) \mathcal{M} \Delta_{i}(1)$ and $v^{*} v \in \Delta_{j}(M)^{\prime} \cap \Delta_{j}(1) \mathcal{M} \Delta_{j}(1)$.

We can now prove:

Theorem 10.7. Let $M$ be a property (T) $I I_{1}$ factor. Then

(1) There exist at most countably many non-conjugate, free ergodic p.m.p. actions $\Gamma \curvearrowright X$ such that $M \cong L^{\infty}(X) \rtimes \Gamma$.

(2) The set of $t>0$ such that $M^{t}$ is isomorphic to the von Neumann algebra of a free ergodic p.m.p. action is countable.

(3) The set of $t>0$ such that $M^{t}$ is isomorphic to a group von Neumann algebra is countable.

Part (3) has been first proven in [38, Section 4. Below, we give a proof relying on a completely different argument.

Proof. (1) Assume by contradiction that there exists an uncountable set $I$ of mutually non-conjugate, free ergodic actions $\Gamma_{i} \curvearrowright X_{i}$ such that $M=L^{\infty}\left(X_{i}\right) \rtimes \Gamma_{i}$. For $i \in I$, define $\Delta_{i}: M \rightarrow M \bar{\otimes} M$ by $\Delta_{i}\left(a u_{\gamma}\right)=a u_{\gamma} \otimes u_{\gamma}$, for all $a \in L^{\infty}\left(X_{i}\right)$ and every $\gamma \in \Gamma_{i}$.

By Lemma 10.6 we can find an uncountable set $J \subset I$ such that for every $i, j \in J$, there exist $0 \neq v \in M \bar{\otimes} M$ satisfying $\Delta_{i}(x) v=v \Delta_{j}(x)$, for all $x \in M$. Since $\Delta_{i}(a)=a$, for all $a \in L^{\infty}\left(X_{i}\right)$, we get that $\Delta_{j}\left(L^{\infty}\left(X_{i}\right)\right) \prec_{M \bar{\otimes} M} M \otimes 1$.

Lemma 9.2 then gives that $L^{\infty}\left(X_{i}\right) \prec_{M} L^{\infty}\left(X_{j}\right)$. Since $L^{\infty}\left(X_{i}\right)$ and $L^{\infty}\left(X_{j}\right)$ are Cartan subalgebras of $M$, 35, Theorem A.1 implies that they are unitarily conjugate. Therefore, the actions $\Gamma_{i} \curvearrowright X_{i}$ and $\Gamma_{j} \curvearrowright X_{j}$ are orbit equivalent ([43], [13]). Since $M=L^{\infty}\left(X_{i}\right) \rtimes \Gamma_{i}$ has property (T), $\Gamma_{i}$ also has property (T), for every $i \in I$. Altogether, we have found an uncountable family of mutually non-conjugate, orbit equivalent, free ergodic actions of property $(\mathrm{T})$ groups. This contradicts [1], Corollary 6.3.

(2) Since property $(\mathrm{T})$ for $\mathrm{II}_{1}$ factors is closed under amplifications, it suffices to show that the set $I=\{\tau(p) \mid p M p$ is the von Neumann algebra of a free, ergodic action $\}$ is countable. Assume by contradiction that $I$ is uncountable. For every $t \in I$, let $p_{t} \in M$ be a projection of trace $t$ and let $\Gamma_{t} \curvearrowright X_{t}$ be a free, ergodic action such that $p_{t} M p_{t}=L^{\infty}\left(X_{t}\right) \rtimes \Gamma_{t}$. Define $\theta_{t}: p_{t} M p_{t} \rightarrow p_{t} M p_{t} \bar{\otimes} p_{t} M p_{t}$ by $\theta_{t}\left(a u_{\gamma}\right)=a u_{\gamma} \otimes u_{\gamma}$, for every $a \in L^{\infty}\left(X_{t}\right)$ and $\gamma \in \Gamma_{t}$.

By taking amplifications we get a $*$-homomorphism $\Delta_{t}: M \rightarrow M \bar{\otimes} M$ satisfying $\tau\left(\Delta_{t}(1)\right)=t$. Since $I$ is uncountable, Lemma 10.6 gives an uncountable set $J \subset I$ such that for every $s, t \in J$ there is $0 \neq v \in \Delta_{t}(1)(M \bar{\otimes} M) \Delta_{s}(1)$ satisfying $\Delta_{t}(x) v=$ $v \Delta_{s}(x)$, for all $x \in M$.

By reasoning similarly to part (1), it follows that the actions $\Gamma_{t} \curvearrowright X_{t}$ and $\Gamma_{s} \curvearrowright X_{s}$ are stably orbit equivalent, for all $s, t \in J$. More precisely, if $\mathcal{R}_{t}$ denotes the equivalence relation induced by $\Gamma_{t} \curvearrowright X_{t}$, then $\mathcal{R}_{t}^{\frac{1}{t}} \cong \mathcal{R}_{s}^{\frac{1}{s}}$, for all $s, t \in J$. Here, $\mathcal{R}^{t}$ denotes the $t$-amplification of an equivalence relation $\mathcal{R}$ (for the definition, see e.g. [15], Section 4). Thus, if we fix $t \in J$, then $\mathcal{R}_{t}^{\frac{s}{t}} \cong \mathcal{R}_{s}$, for every $s \in J$. On the other hand, [15, Theorem 5.9 gives that the set $\left\{q>0 \mid \mathcal{R}_{t}^{q}\right.$ is induced by a free action of a countable group $\}$ is countable. Thus, $J$ must be countable, a contradiction.

(3) It suffices to prove that $I=\{\tau(p) \mid p M p$ is a group von Neumann algebra $\}$ is countable. For $t \in I$, let $p_{t} \in M$ be a projection of trace $t$ and let $\Gamma_{t}$ be a group such 
that $p_{t} M p_{t}=L\left(\Gamma_{t}\right)$. Let $\theta_{t}: L\left(\Gamma_{t}\right) \rightarrow L\left(\Gamma_{t}\right) \bar{\otimes} L\left(\Gamma_{t}\right)$ be given by $\theta_{t}\left(u_{\gamma}\right)=u_{\gamma} \otimes u_{\gamma}$, for each $\gamma \in \Gamma_{t}$.

Since $M$ is a factor, $\Gamma_{t}$ is ICC. Thus, the set $\left\{\left(\gamma g \gamma^{-1}, \gamma h \gamma^{-1}\right) \mid \gamma \in \Gamma_{t}\right\}$ is infinite, for all $(g, h) \in\left(\Gamma_{t} \times \Gamma_{t}\right) \backslash\{(e, e)\}$. This fact implies that $\theta_{t}\left(L\left(\Gamma_{t}\right)\right)^{\prime} \cap L\left(\Gamma_{t}\right) \bar{\otimes} L\left(\Gamma_{t}\right)=$ $\mathbb{C} 1$, for all $t$. Since $p_{t} M p_{t}=L\left(\Gamma_{t}\right)$, by amplifying $\theta_{t}$ we get a $*$-homomorphism $\Delta_{t}$ : $M \rightarrow M \bar{\otimes} M$ verifying $\tau\left(\Delta_{t}(1)\right)=t$ and $\Delta_{t}(M)^{\prime} \cap \Delta_{t}(1)(M \bar{\otimes} M) \Delta_{t}(1)=\mathbb{C} \Delta_{t}(1)$.

Finally, Lemma 10.6 implies that $I=\left\{\tau\left(\Delta_{t}(1)\right) \mid t \in I\right\}$ is countable.

Remarks 10.8. (1) Let $M$ be a $\mathrm{II}_{1}$ factor. Recall that every left-finite $M$-bimodule is of the form $\mathcal{H}_{\theta}$, for some unital $*$-homomorphism $\theta: M \rightarrow M^{t}$. Notice that $\mathcal{H}_{\theta}$ is irreducible iff $\theta(M)^{\prime} \cap M^{t}=\mathbb{C} 1$. Lemma 10.6 thus implies that if $M$ has property $(\mathrm{T})$, then there are only countably many irreducible left-finite Hilbert $M$-bimodules (up to isomorphism).

(2) $\mathrm{A} \mathrm{II}_{1}$ factor $M$ is solid if $A^{\prime} \cap M$ is atomic, for any completely non-amenable von Neumann subalgebra $A \subset M$. N. Ozawa proved that $L(\Gamma)$ is solid, for any hyperbolic group $\Gamma$ ([27]). If $M$ is a non-amenable solid factor, then $\theta(M)^{\prime} \cap M^{t}$ is atomic, for every unital $*$-homomorphism $\theta: M \rightarrow M^{t}$. This fact implies that any left-finite $M$-bimodule is the direct sum of countably many irreducible left-finite $M$-bimodules.

(3) Let us explain how (1) and (2) imply that there are $\mathrm{II}_{1}$ factors $M$ with "few" left-finite bimodules. Indeed, take $M=L(\Gamma)$, for some hyperbolic, property ( $\mathrm{T}$ ) group $\Gamma$. Combining (1) and (2) yields that there exist a countable family of Hilbert $M$-bimodules $\left\{\mathcal{H}_{n}\right\}_{n \geq 1}$ such that every left-finite Hilbert $M$-bimodule $\mathcal{H}$ is the direct sum of some of the $\mathcal{H}_{n}$ 's.

(4) Finally, note that N. Ozawa has very recently shown that if $\Gamma$ is a hyperbolic, property (T), ICC group (e.g. any ICC lattice $\Gamma<\operatorname{Sp}(1, n))$, then $L(\Gamma)$ is a property (T) $\mathrm{II}_{1}$ factor which does not admit a group measure space decomposition (29]).

(IV) $\mathbf{I I}_{1}$ factors not isomorphic to twisted group von Neumann algebras. We end the paper by noticing that an extension of the results from Section 8 can be used to give examples of $\mathrm{II}_{1}$ factors that are not isomorphic to twisted group von Neumann algebras.

Let us begin by recalling the construction of the von Neumann algebra $L_{\alpha}(G)$ arising from a countable group $G$ and a 2 -cocycle $\alpha \in \mathrm{H}^{2}(G, \mathbb{T})$, i.e. a map $\alpha$ : $G \times G \rightarrow \mathbb{T}$ satisfying $(\diamond) \alpha(g, h) \alpha(g h, k)=\alpha(g, h k) \alpha(h, k)$, for all $g, h, k \in G$.

First, the formula

$$
u_{g}^{\alpha}\left(\delta_{h}\right)=\alpha(g, h) \delta_{g h}, \forall g, h \in G
$$

defines a projective unitary representation $u^{\alpha}: G \rightarrow \mathcal{U}\left(\ell^{2} G\right)$, where $\left\{\delta_{h}\right\}_{h \in G}$ is the usual orthonormal basis of $\ell^{2} G$. More precisely, $u_{g}^{\alpha} u_{h}^{\alpha}=\alpha(g, h) u_{g h}^{\alpha}$, for all $g, h \in G$.

Then $L_{\alpha}(G)$ is defined as the von Neumann algebra generated by $\left\{u_{g}^{\alpha}\right\}_{g \in G}$. Note that $\tau: L_{\alpha}(G) \rightarrow \mathbb{C}$ given by $\tau\left(u_{g}^{\alpha}\right)=\delta_{g, e} \alpha(e, e)$ is a faithful normal trace.

We continue with two useful facts about twisted group von Neumann algebras.

Lemma 10.9. Let $G$ be a countable group and $\alpha \in H^{2}(G, \mathbb{T})$ be a 2-cocycle. Let $\bar{\alpha} \in H^{2}(G, \mathbb{T})$ be given by $\bar{\alpha}(g, h)=\overline{\alpha(g, h)}$, for all $g, h \in G$. Then

(1) The opposite von Neumann algebra $L_{\alpha}(G)^{\text {op }}$ is isomorphic to $L_{\bar{\alpha}}(G)$.

(2) The map $\Delta: G \rightarrow L_{\alpha}(G) \bar{\otimes} L_{\alpha}(G) \bar{\otimes} L_{\bar{\alpha}}(G)$ given by $\Delta\left(u_{g}^{\alpha}\right)=u_{g}^{\alpha} \otimes u_{g}^{\alpha} \otimes u_{g}^{\bar{\alpha}}$, for every $g \in G$, extends to a unital $*$-homomorphism $\Delta: L_{\alpha}(G) \rightarrow L_{\alpha}(G) \bar{\otimes} L_{\alpha}(G) \bar{\otimes}$ $L_{\bar{\alpha}}(G)$. 
Proof. (1) Denote $\beta=\alpha(e, e)$ and notice that the cocycle identity $(\diamond)$ implies that $\alpha(e, g)=\beta$, for all $g \in G$.

Now, for every $g \in G$, define $\theta\left(u_{g}^{\alpha}\right)=\beta \alpha\left(g, g^{-1}\right) u_{g^{-1}}^{\bar{\alpha}} \in L_{\bar{\alpha}}(G)$.

We claim that $\theta$ extends to a $*$-isomorphism $\theta: L_{\alpha}(G)^{o p} \rightarrow L_{\bar{\alpha}}(G)$. To see this, it suffices to prove that $\theta$ is trace preserving and that $\theta\left(u_{h}^{\alpha} u_{g}^{\alpha}\right)=\theta\left(u_{g}^{\alpha}\right) \theta\left(u_{h}^{\alpha}\right)$, for all $g, h \in G$. Fix $g, h \in G$.

The first assertion holds because $\tau\left(\theta\left(u_{g}^{\alpha}\right)\right)=\beta \alpha\left(g, g^{-1}\right) \delta_{g, e} \overline{\alpha(e, e)}=\delta_{g, e} \beta=$ $\tau\left(u_{g}^{\alpha}\right)$.

For the second assertion, using the cocycle identity twice yields that

$$
\begin{gathered}
\alpha(h, g) \alpha\left(h g, g^{-1} h^{-1}\right) \alpha\left(g^{-1}, h^{-1}\right)=\alpha\left(h, h^{-1}\right) \alpha\left(g, g^{-1} h^{-1}\right) \alpha\left(g^{-1}, h^{-1}\right) \\
=\alpha\left(e, h^{-1}\right) \alpha\left(g, g^{-1}\right) \alpha\left(h, h^{-1}\right) .
\end{gathered}
$$

Since

$$
\theta\left(u_{h}^{\alpha} u_{g}^{\alpha}\right)=\alpha(h, g) \theta\left(u_{h g}^{\alpha}\right)=\beta \alpha(h, g) \alpha\left(h g, g^{-1} h^{-1}\right) u_{g^{-1} h^{-1}}^{\bar{\alpha}},
$$

while

$$
\begin{aligned}
\theta\left(u_{g}^{\alpha}\right) \theta\left(u_{h}^{\alpha}\right) & =\beta^{2} \alpha\left(g, g^{-1}\right) \alpha\left(h, h^{-1}\right) u_{g^{-1}}^{\bar{\alpha}} u_{h^{-1}}^{\bar{\alpha}} \\
& =\beta^{2} \alpha\left(g, g^{-1}\right) \alpha\left(h, h^{-1}\right) \overline{\alpha\left(g^{-1}, h^{-1}\right)} u_{g^{-1} h^{-1}}^{\bar{\alpha}},
\end{aligned}
$$

the second assertion follows by using (10.a).

(2) Since $\Delta_{\mid G}$ is clearly multiplicative and trace preserving, the conclusion follows.

Next, let us point out a generalization of the results from Section 8. Let $\Gamma$ be a torsion free, ICC group which admits an infinite almost normal subgroup such that the inclusion $\left(\Gamma_{0} \subset \Gamma\right)$ has the relative property $(\mathrm{T})$. Let $B$ be a non-trivial abelian von Neumann algebra and define $M=B^{\Gamma} \rtimes \Gamma$.

Theorems 8.1 and 8.2 classify embeddings of $M$ into itself and into $M \bar{\otimes} M$, repsectively. A straightforward modification of the proof of Theorem 8.2 shows that the embeddings of $M$ into $\bar{\bigotimes}_{i=1}^{n} M$ can be classified in a similar way, for every $n \geqslant 1$.

Theorem 10.10. Let $M=B^{\Gamma} \rtimes \Gamma$ be as above and denote $A=B^{\Gamma}$. Let $\Delta: M \rightarrow$ $M^{S}$ be a $*$-homomorphism, for some finite set $S$, and suppose that:

(1) $\Delta\left(L\left(\Gamma_{0}\right)\right) \nprec_{M^{S}} L(\Gamma)^{S \backslash\{s\}}$, for any $s \in S$.

(2) $\Delta(M) \nprec_{M^{S}} L(\Gamma)^{\{s\}} \bar{\otimes} M^{S \backslash\{s\}}$, for any $s \in S$.

Then $\Delta$ must be unital and we can find a character $\eta$ of $\Gamma$, group homomorphisms $\delta_{s}: \Gamma \rightarrow \Gamma$, for every $s \in S$, and a unitary $u \in M^{S}$ such that $u \Delta(A) u^{*} \subset A^{S}$ and $u \Delta\left(u_{\gamma}\right) u^{*}=\eta(\gamma)\left(\bigotimes_{s \in S} u_{\delta_{s}(\gamma)}\right)$, for every $\gamma \in \Gamma$.

Here we denote by $M^{S}$ the tensor product algebra $\bar{\bigotimes}_{s \in S}(M)_{s}$. For a subset $S^{\prime} \subset S$ and a von Neumann subalgebra $Q \subset M$, we view $Q^{S^{\prime}}$ as a von Neumann subalgebra of $M^{S}$, in the obvious way.

Finally, let us combine the last two results to give examples of $\mathrm{II}_{1}$ factors which are not isomorphic to twisted group von Neumann algebras.

Corollary 10.11. Let $M$ be as above and $p \in M \backslash\{1\}$ be a projection. Then $p M p$ is not isomorphic to $L_{\alpha}(G)$, for any countable group $G$ and any $\alpha \in H^{2}(G, \mathbb{T})$. 
Proof. Assume that $p M p$ can be written as $L_{\alpha}(G)$, for some projection $p \in M$. Our goal is to prove that $p=1$. First, by Remark $10.3(3)$, we have that $p M p$ is anti-isomorphic to itself, i.e. $(p M p)^{o p} \cong p M p$. On the other hand, part (1) of Lemma 10.9 gives that $L_{\alpha}(G)^{o p} \cong L_{\bar{\alpha}}(G)$. Altogether, we deduce the existence of a *-isomorphism $\theta: L_{\bar{\alpha}}(G) \rightarrow L_{\alpha}(G)$.

Next, let $S=\{1,2,3\}$ and consider the $*$-homomorphism

$$
\Delta_{1}:=(i d \otimes i d \otimes \theta) \circ \Delta: L_{\alpha}(G) \rightarrow L_{\alpha}(G)^{S}=\bar{\bigotimes}_{i=1}^{3} L_{\alpha}(G),
$$

where $\Delta$ is as in Lemma $10.9(2)$.

Concretely, we have that $\Delta_{1}\left(u_{g}^{\alpha}\right)=u_{g}^{\alpha} \otimes u_{g}^{\alpha} \otimes \theta\left(u_{g}^{\bar{\alpha}}\right)$, for all $g \in G$.

To simplify notation, denote $N=L_{\alpha}(G)$. Since $\Delta_{1}$ comes from a "diagonal embedding" of $G$, we have the following:

Claim. Let $Q \subset N$ be a von Neumann subalgebra.

(1) If $\Delta_{1}(Q) \prec_{N^{S}} N^{S \backslash\{s\}}$, for some $s \in S$, then $Q$ is not diffuse.

(2) If $\Delta_{1}(N) \prec_{N S} Q^{\{s\}} \bar{\otimes} N^{S \backslash\{s\}}$, then $N \prec_{N} Q$.

The proof of this claim is analogous to the proof of Lemma 9.2 and so we leave the details to the reader.

Now, since $N=p M p$, by amplifying $\Delta_{1}: N \rightarrow N^{S}$, we get a $*$-homomorphism $\Delta_{2}: M \rightarrow M^{S}$, such that $\tau\left(\Delta_{2}(1)\right)=\tau(p)^{2}$. By using the claim it is immediate that $\Delta_{2}$ satisfies conditions (1) and (2) in Theorem 10.10. Thus, by applying Theorem 10.10 it follows that $\Delta_{2}$ is unital; hence $p=1$.

Acknowledgments. I would like to thank Jesse Peterson for explaining 31] and Sorin Popa for many useful suggestions. I am also grateful to Vaughan Jones for bringing to my attention the question that led to Corollary G, Stefaan Vaes for providing me with an argument which simplifies the initial proof of Theorem 9.1 and the anonymous referee for several suggestions which helped improve the exposition. This paper was written while I was visiting the Department of Mathematics at UCLA.

\section{REFERENCES}

[1] B. Bekka, P. de la Harpe, A. Valette: Kazhdan's property (T), New Mathematical Monographs, 11. Cambridge University Press, Cambridge, 2008. MR2415834 (2009i:22001)

[2] N.P. Brown, N. Ozawa: $C^{*}$-algebras and finite-dimensional approximations, Graduate Studies in Mathematics, 88. American Mathematical Society, Providence, RI, 2008. xvi+509 pp. MR2391387 (2009h:46101)

[3] M. Burger: Kazhdan constants for SL(3, Z), J. Reine Angew. Math. 413 (1991), 36-67. MR:1089795 (92c:22013)

[4] A. Connes: Sur la classification des facteurs de type II, C. R. Acad. Sci. Paris 281 (1975), 13-15. MR0377534 (51:13706)

[5] A. Connes: Correspondences, handwritten notes, 1980.

[6] A. Connes: A factor of type $I I_{1}$ with countable fundamental group, J. Operator Theory 4 (1980), no. 1, 151-153. MR.587372 (81j:46099)

[7] A. Connes, J. Feldman, B. Weiss: An amenable equivalence relation is generated by a single transformation, Ergodic Theory and Dynam. Systems 1 (1981), no. 4, 431-450. MR662736 (84h:46090)

[8] I. Chifan, A. Ioana: Ergodic Subequivalence Relations Induced by a Bernoulli Action, Geom. Funct. Anal. Vol. 20 (2010) 53-67. MR2647134(2011f:37008)

[9] A. Connes, V.F.R. Jones: A II factor with two non-conjugate Cartan subalgebras, Bull. Amer. Math. Soc. 6 (1982), 211-212. MR640947 (83d:46074) 
[10] A. Connes, V.F.R. Jones: Property (T) for von Neumann algebras, Bull. London Math. Soc. 17 (1985), 57-62. MR766450 (86a:46083)

[11] A. Furman: Orbit equivalence rigidity, Ann. of Math. (2) 150 (1999), 1083-1108. MR 1740985 (2001a:22018)

[12] A. Furman: A survey of Measured Group Theory, Geometry, Rigidity, and Group Actions, 296-374, The University of Chicago Press, Chicago and London, 2011, available at arXiv:0901.0678.

[13] J. Feldman, C.C. Moore: Ergodic equivalence relations, cohomology, and von Neumann algebras, II, Trans. Amer. Math. Soc. 234 (1977), 325-359. MR0578730 (58:28261b)

[14] A. Ioana: Rigidity results for wreath product II 1 factors, J. Funct. Anal. 25 (2007) 763-791. MR2360936 (2008j:46046)

[15] A. Ioana: Cocycle superrigidity for profinite actions of property (T) groups, Duke Math. J. Volume 157, Number 2 (2011), 337-367.

[16] A. Ioana: Non-orbit equivalent actions of $\mathbb{F}_{n}$, Ann. Sci. Éc. Norm. Supér., 42, fascicule 4 (2009), 675-696. MR2568879 (2010k:37007)

[17] A. Ioana, J. Peterson, S. Popa: Amalgamated free products of weakly rigid factors and calculation of their symmetry groups, Acta Math. 200 (2008), no. 1, 85-153. MR.2386109 (2009a:46119)

[18] V.F.R. Jones: A II factor anti-isomorphic to itself but without involutory antiautomorphisms, Math. Scand. 46 (1980), no. 1, 103-117. MR585235(82a:46075)

[19] R.V. Kadison, J.R. Ringrose: Fundamentals of the theory of operator algebras. Vol. II, Academic Press, Orlando, 1986. MR859186 (88d:46106)

[20] D. Kazhdan: Connection of the dual space of a group with the structure of its closed subgroups, Funct. Anal. and its Appl., 1 (1967), 63-65. MR0209390 (35:288)

[21] Y. Kida: Measure equivalence rigidity of the mapping class group, Ann. of Math. (2) 171 (2010), No. 3, 1851-1901. MR2680399 (2011e:37009)

[22] Y. Kida: Rigidity of amalgamated free products in measure equivalence theory, to appear in J. Topology, preprint arXiv:0902.2888.

[23] G. Margulis: Finitely-additive invariant measures on Euclidean spaces, Ergodic Theory and Dynam. Systems 2 (1982), 383-396. MR721730 (85g:28004)

[24] F. Murray, J. von Neumann: On rings of operators, Ann. of Math. (2) 37 (1936), 116-229. MR.1503275

[25] F. Murray, J. von Neumann: On rings of operators. IV, Ann. of Math. (2) 44 (1943), 716-808. MR0009096 (5:101a)

[26] D. Ornstein, B. Weiss: Ergodic theory of amenable groups. I. The Rokhlin lemma., Bull. Amer. Math. Soc. (N.S.) 1 (1980), 161-164. MR551753 (80j:28031)

[27] N. Ozawa: Solid von Neumann algebras, Acta Math. 192 (2004), no. 1, 111-117. MR2079600 (2005e:46115)

[28] N. Ozawa, S. Popa: On a class of $I I_{1}$ factors with at most one Cartan subalgebra, Ann. of Math. (2) Vol. 172 (2010), No. 1, 713-749. MR2680430

[29] N. Ozawa: Examples of groups which are not weakly amenable, preprint arXiv:1012.0613.

[30] J. Peterson: $L^{2}$-rigidity in von Neumann algebras, Invent. Math. 175 (2009), no. 2, 417-433. MR2470111 (2010b:46128)

[31] J. Peterson: Examples of group actions which are virtually $W^{*}$-superrigid, preprint arXiv:1002.1745.

[32] S. Popa: Correspondences, INCREST preprint 1986, unpublished.

[33] S. Popa: Strong Rigidity of II 1 Factors Arising from Malleable Actions of w-Rigid Groups. I., Invent. Math. 165 (2006), 369-408. MR2231961 (2007f:46058)

[34] S. Popa: Strong Rigidity of $I_{1}$ Factors Arising from Malleable Actions of w-Rigid Groups. II., Invent. Math. 165 (2006), 409-451. MR2231962 (2007h:46084)

[35] S. Popa: On a class of type II $I_{1}$ factors with Betti numbers invariants, Ann. of Math. (2) 163 (2006), 809-889. MR2215135 (2006k:46097)

[36] S. Popa: Some rigidity results for non-commutative Bernoulli shifts, J. Funct. Anal. 230 (2006), 273-328. MR2186215 (2007b:46106)

[37] S. Popa: Cocycle and orbit equivalence superrigidity for malleable actions of w-rigid groups, Invent. Math. 170 (2007), no. 2, 243-295. MR2342637(2008f:37010) 
[38] S. Popa: Deformation and rigidity for group actions and von Neumann algebras, International Congress of Mathematicians. Vol. I, 445-477, Eur. Math. Soc., Zürich, 2007. MR2334200 (2008k:46186)

[39] S. Popa: On the superrigidity of malleable actions with spectral gap, J. Amer. Math. Soc. 21 (2008), 981-1000. MR2425177 (2009e:46056)

[40] S. Popa, S. Vaes: Strong rigidity of generalized Bernoulli actions and computations of their symmetry groups. Adv. Math. 217 (2008), no. 2, 833-872. MR2370283 (2009c:37004)

[41] S. Popa, S. Vaes: On the fundamental group of $I I_{1}$ factors and equivalence relations arising from group actions, Quanta of maths, 519-541, Clay Math. Proc., 11, Amer. Math. Soc., Providence, RI, 2010. MR2732063

[42] S. Popa, S. Vaes: Group measure space decomposition of $I I_{1}$ factors and $W^{*}$-superrigidity, Invent. Math. 182 (2010), no. 2, 371-417. MR.2729271

[43] I.M. Singer: Automorphisms of finite factors, Amer. J. Math. 77 (1955), 117-133. MR0066567 (16:597f)

[44] S. Vaes: Rigidity results for Bernoulli actions and their von Neumann algebras (after Sorin Popa), Séminaire Bourbaki. Vol. 2005/2006. Astérisque No. 311 (2007), Exp. No. 961, viii, 237-294. MR2359046 (2009k:46112)

[45] S. Vaes: Explicit computations of all finite index bimodules for a family of $I I_{1}$ factors, Ann. Sci. Éc. Norm. Supér. (4) 41 (2008), no. 5, 743-788. MR2504433 (2010k:46061)

[46] S. Vaes: Factors of type II $I_{1}$ without non-trivial finite index subfactors, Trans. Amer. Math. Soc. 361 (2009), no. 5, 2587-2606. MR2471930(2010a:46146)

Department of Mathematics, UCla, Los Angeles, California 91125 and imar, 21 Calea Grivitei Street, 010702 Bucharest, Romania

E-mail address: adiioana@math.ucla.edu 